\title{
Long-period variables in NGC 147 and NGC 185^
}

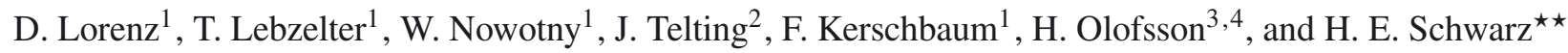 \\ 1 University of Vienna, Department of Astronomy, Türkenschanzstrasse 17, 1180 Vienna, Austria \\ e-mail: denise.lorenz@univie.ac.at \\ 2 Nordic Optical Telescope, Apartado 474, 38700 Santa Cruz de La Palma, Spain \\ 3 Department of Astronomy, Stockholm University, AlbaNova University Center, 10691 Stockholm, Sweden \\ ${ }^{4}$ Onsala Space Observatory, 43992 Onsala, Sweden
}

Received 24 March 2011 / Accepted 25 May 2011

\begin{abstract}
Context. Previous studies on the stellar content of the two nearby dwarf galaxies NGC 147 and NGC 185 reveal a rich population of late-type giants in both systems, including a large number of carbon-rich objects. These stars are known to show pronounced photometric variability, which can be used for a more detailed characterisation of these highly evolved stars. Owing to their well-studied parameters, these Local Group members are ideal candidates for comparative studies.

Aims. Through photometric monitoring, we attempt to provide a catalogue of long-period variables (LPVs), including Mira variables, semi-regular variables, and even irregular variables in NGC 147 and NGC 185. We investigate the light variations and compare the characteristics of these two LPV populations with the results found for other galaxies, such as the LMC.

Methods. We carried out time-series photometry in the $i$-band of the two target galaxies with the Nordic Optical Telescope (NOT), covering a time span of $\approx 2.5$ years. More than 30 epochs were available for a period search. These data were then combined with single-epoch $K$-band photometry, also obtained with the NOT. Narrow-band photometry data from the literature was used to distinguish between $\mathrm{O}$-rich and $\mathrm{C}$-rich stars.

Results. We report the detection of 513 LPVs in NGC 185 and 213 LPVs in NGC 147, showing amplitudes $\Delta i$ of up to $\approx 2^{\text {mag }}$ and periods ranging between 90 and 800 days. The period-luminosity diagram for each of our target galaxies exhibits a well populated sequence of fundamental mode pulsators. The resulting period-luminosity relations we obtained are compared to relations from the literature. We discuss the universality of those relations because of which, as a side result, a correction of the distance modulus of NGC 185 may be necessary. A value of $(m-M)=24$ m. 30 seems to be more appropriate to match the observed data. Only one of our two galaxies, namely NGC 185, has a significant fraction of possibly first overtone pulsators. An interpretation of this finding in terms of differences in the star-formation histories is suggested.
\end{abstract}

Key words. stars: AGB and post-AGB - stars: late-type - stars: variables: general - Local Group - galaxies: individual: NGC 147 galaxies: individual: NGC 185

\section{Introduction}

Asymptotic giant branch (AGB) stars are highly evolved stars with low to intermediate initial masses of $\approx 0.6-8 M_{\odot}$ that have passed the helium-core burning phase. These stars are then powered by nuclear burning of hydrogen and helium in two thin shells on top of a core of carbon $(\mathrm{C})$ and oxygen $(\mathrm{O})$. During the early AGB phase these stars are O-rich, showing a photospheric $\mathrm{C} / \mathrm{O}$-ratio $<1$, and most of them can be classified as stars of spectral type M. For AGB stars with initial masses up to $\approx 4 M_{\odot}$ the atmospheric chemical composition can change dramatically because processed elements, most notably ${ }^{12} \mathrm{C}$, are dredged up to the surface by convective mixing after a thermal pulse. Depending on the C/O-ratio, their spectral type changes from $\mathrm{K}$ or $\mathrm{M}$ via $\mathrm{S}$ to $\mathrm{C}(\mathrm{C} / \mathrm{O} \geq 1)$ (Groenewegen 2007). For AGB stars with initial masses $\gtrsim 4 M_{\odot}$ the temperature at the bottom of the convective envelope rises sufficiently high to transform $\mathrm{C}$ into N. This process, called hot-bottom-burning, causes some AGB star to remain O-rich.

\footnotetext{
* Appendix is available in electronic form at

http: //www . aanda.org

$\star \star$ Deceased, 2006 October 20.
}

Long-period variables (LPVs) is the generic term for variable stars known as Mira variables and semi-regular variables. They generally show periodic variations in brightness with periods of $\approx 30$ up to a few thousands of days and amplitudes ranging from several tenths to approximately ten magnitudes in the visual. By studying LPVs in the Large Magellanic Cloud (LMC), Wood et al. (1999) and Wood (2000) were the first to discover that all LPVs seem to group around at least five almost parallel sequences in a period-luminosity-diagram (PLD), to which the authors assigned the letters A to E. Sequence C was explained as consisting of stars that pulsate in fundamental mode. Longperiod variables on sequence B were explained as first and second overtone pulsators, and stars on sequence $\mathrm{A}$ as higher overtone variables. Later publications of period-luminosity relations (PLRs) of the LMC revealed that sequence B actually splits into two separate sequences, namely B and $\mathrm{C}^{\prime}$ (see Kiss \& Bedding 2003; Ita et al. 2004). Meanwhile, different authors (e.g. Fraser et al. 2005; Soszyński et al. 2004a) are using different labels in a PLD; we use the original labelling of Wood et al. (1999) plus the additional sequence $\mathrm{C}^{\prime}$. For stars belonging to sequences $\mathrm{E}$ or $\mathrm{D}$ the variability cannot be attributed to radial pulsation. Stars on sequence $\mathrm{E}$ are thought to be related to close binary systems 
showing ellipsoidal variability (see Soszyńsky et al. 2004b). Recently, Nicholls et al. (2010) confirmed this assumption by comparing the phased light and radial velocity curves of LMC red giant binaries. The results of this study also demonstrated that the variations of stars on sequence $E$ and $D$ are caused by different mechanisms. The LPVs on sequence D show periodicites on two time scales, where the long secondary period (LSP) is about ten times the shorter period. The origin of these LSPs is still unknown (Nicholls et al. 2009). By comparing a sample of sequence D stars with similar red giants (not showing LSPs), Wood \& Nicholls (2009) found that such objects have a significant excess in the mid-infrared $(8-24 \mu \mathrm{m})$, which is thought to originate from circumstellar dust. This is not the case for sequence E stars (Nicholls et al. 2010). Within the last decade, several studies were carried out to explore PLRs of LPVs in different stellar systems of the Local Group (see Groenewegen 2005 for an overview). Rejkuba et al. (2003) and Rejkuba (2004) were able to study LPVs even beyond the Local Group, namely in NGC 5128 in the Centaurus group. This growing sample of PLRs for LPVs raises the question whether these relations are universal or not and if they are indeed universal, they can, therefore, be used as an additional tool to measure distances. Here we aim to contribute to this discussion by investigating LPVs in the two dwarf galaxies NGC 147 and NGC 185.

The two target galaxies of our investigation, NGC 147 and NGC 185, were discovered by J. Herschel in September 1829 and by W. Herschel in November 1787, respectively, and are known to be members of the M31 subgroup. Together with NGC 205, they are the most luminous dSphs in the Local Group and are located at an angular distance of approximately $12^{\circ}$ from the Andromeda nebula (van den Bergh 1998; Corradi 2005). According to van den Bergh (1998), they are separated by only 58 ' on the sky without any indication of interaction (Battinelli \& Demers 2004a; Geha et al. 2010). Although these galaxies appear fairly similar concerning their colour-magnitude diagrams (CMDs), some differences can be found in their star formation histories (SFHs), most notably for recent epochs ( $<1$ Gyr see Mateo 1998). Because there are no main-sequence turn-off stars with $M_{V}<-1$, Han et al. (1997) mentioned that the most recent large-scale star-forming activity in NGC 147 occurred at least $1 \mathrm{Gyr}$ in the past. According to broad-band near-infrared CMDs of Riebel (2010), this event happened $\approx 3$ Gyr ago and Dolphin (2000) derived 4 Gyr using HST images. That there are no signs of dust and gas (Young \& Lo 1997; Sage 1994) in this galaxy, which could serve as building material for new stars, also supports the idea that star formation ceased long ago. Using the relation between the $K$-magnitude of the AGB tip and age (as predicted from isochrones; Girardi et al. 2002), Sohn et al. (2006) find that most of the M-giants in NGC 147 formed between at $\log \left(t_{\mathrm{yr}}\right)$ between 8.2-8.6. In contrast, various authors found a significant amount of gas and dust in the centre of NGC 185 (Young \& Lo 1997; Lee et al. 1993; Martinez-Delgado \& Aparicio 1998; Martinez-Delgado et al. 1998). Butler \& Martinez-Delgado (2005) obtained an age of about $400 \mathrm{Myr}$ for the youngest, centrally concentrated stars. Kang et al. (2005) speculate that the M-giant population in NGC 185 contains stars with a wide range of ages, possibly representing two different epochs of star formation at $\log \left(t_{\mathrm{yr}}\right) \approx 9.0-9.4$ and $7.8-8.5$ dex. In the outer parts of NGC 185 stars with ages of at least $1 \mathrm{Gyr}$ are found.

The red giant content of these galaxies was analysed by Nowotny et al. (2003, hereafter Paper I). The detected AGB stars were characterised according to the chemical properties of their atmospheres by applying an efficient method to single out C-rich stars, namely, the use of narrow-band wing-type filters that are centred around spectral molecular features of $\mathrm{TiO}$ and $\mathrm{CN}$ (at $\lambda \approx 0.8 \mu \mathrm{m}$ ). Within a field of view (FOV) of $6.5 \times 6.5$, the authors identified 154 C-rich stars in NGC 185 and 146 C-rich stars in NGC 147 plus several hundred M-Type stars on the upper giant branch in both galaxies. This large number of identified AGB stars motivated a search for LPVs in these stellar systems. An interesting aspect was if the different metallicities and SFHs would be reflected in the PLRs of the LPVs in the two galaxies.

The main aim of this work was to identify LPVs in NGC 147 and NGC 185. In Sect. 3.3 we present a catalogue of red giant variables, the outcome of a photometric monitoring in the $i$-band. The results of this study are summarised in Sect. 4.

\section{Observations}

\subsection{Photometric monitoring}

We obtained multi-epoch observations in the Gunn-i-band with the $2.56 \mathrm{~m}$ Nordic Optical Telescope (NOT). The target galaxies were observed on 38 nights in service mode between October 2003 and February 2006 with the Andalucia Faint Object Spectrograph Camera (ALFOSC). It has a pixel scale of $0.19 \mathrm{arcsec} / \mathrm{pixel}$ resulting in a FOV of approximately $6.4 \times$ $6.4 \mathrm{arcmin}$. At every epoch we obtained a single image pointing towards the centre of each galaxy. Our field covered a region corresponding to approximately one scale length derived from the stellar density distribution of NGC 147 (Battinelli \& Demers 2004b) and NGC 185 (Battinelli \& Demers 2004a), respectively. We obtained 35 images of NGC 147 and 34 frames of NGC 185 with a sampling period of $\approx 14$ days. One example image of the time series for each of our science targets is shown in Fig. 1. The time series exhibits two larger gaps of approximately six months during which the targets were not observable. Calibration frames to correct for sky and bias were recorded for each night of observation. In the rare cases of missing sky flats, these were replaced by average flats from the previous and the following observation.

\section{2. $K_{s}$-band photometry}

As can be seen from spectral energy distributions of cool AGB stars (Nowotny et al. 2010), they emit most of their flux in near-infrared wavelengths. Hence, the $K_{\mathrm{s}}$-band is a good measure of the bolometric flux. The most evolved, dustenshrouded AGB stars can be detected only at infrared wavelengths. Therefore, the $K_{\mathrm{s}}$-band has been widely used (e.g. Wood 2000) to construct PLRs of LPVs. To allow a comparison of our results with previous studies, we carried out single-epoch $K_{\mathrm{s}}$-band photometry for our target systems using NOTCam during two consecutive nights in September 2004. This camera is equipped with a $1024 \times 1024 \mathrm{HgCdTe}$ Rockwell Hawaii array with a plate scale of $0.234 \mathrm{arcsec} /$ pixel resulting in a FOV of $4 \times 4$ arcmin using the wide-field imaging mode of NOTCam. To resemble the FOV of ALFOSC, we obtained a mosaic of four partly overlapping dithered images per galaxy. Accordingly, the combined FOV of the four quadrants is $\approx 6 \times 6$ arcmin.

\section{Data reduction}

\subsection{Monitoring data}

All frames obtained for this study were bias-, sky- and flatfieldcorrected using standard data reduction routines. As in Paper I, 

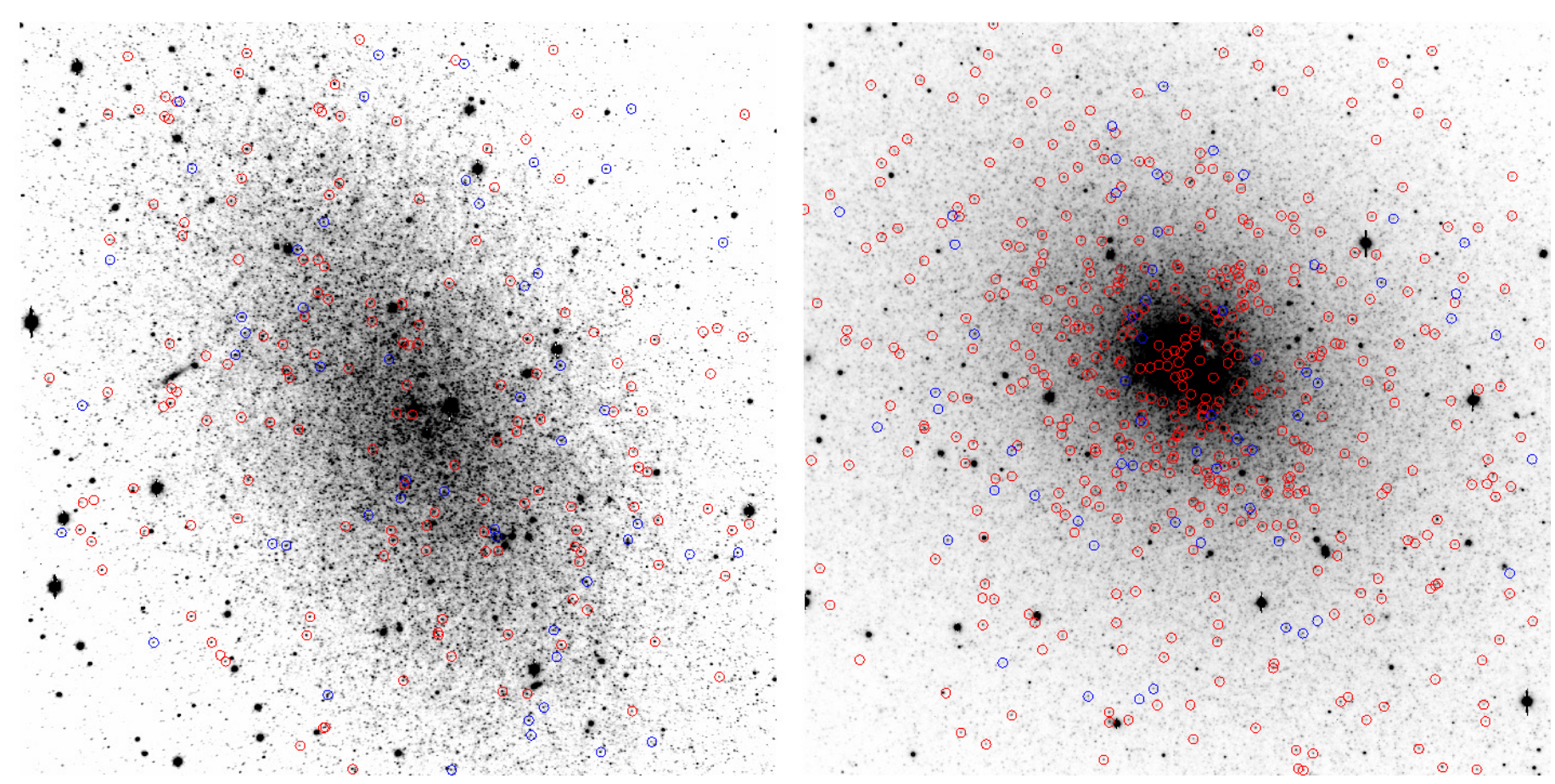

Fig. 1. Inverted CCD images of NGC 147 (left panel) and NGC 185 (right panel) obtained with NOT in the $i$-band. North is up and east is to the left. Designated with blue circles are objects that were identified as LPVs in this work and for which the narrow-band photometry in Paper I indicates C-rich atmospheric chemistry. The red circles mark all other stars (presumably O-rich) that were found to show long-period variability.

the whole sample of stars was corrected for interstellar reddening adopting the values from the NASA Extragalactic Database (NGC 147: $A_{V}=0.574, A_{i}=0.536, A_{K_{\mathrm{s}}}=0.064 ;$ NGC 185: $\left.A_{V}=0.604, A_{i}=0.354, A_{K_{\mathrm{s}}}=0.067\right)$. Images taken in the $i$-band with ALFOSC also suffer from fringing. To compensate for this effect, it would have been necessary to obtain flatfield images before and after each integration of the science target to create a fringe map. Without these additional calibration images, a correction for this effect was not possible. The maximum amplitude of variations caused by fringing is, however, well below the minimum amplitude expected for LPVs. The detection of variable stars was carried out using the image subtraction tool ISIS $2.1^{1}$ of Alard (2000). One carefully chosen $i$-band image was taken as reference frame to obtain differences in flux relative to each image of the time series. To produce light curves from these differences, we measured fluxes for each star on the reference frame by using a PSF fitting software written by $\mathrm{Ch}$. Alard. Short descriptions of the code, which was originally developed for the DENIS project, can be found in Schuller et al. (2003) or Beaulieu et al. (2008). As can be seen in Fig. 1, the central region of NGC 185 is more compact towards the centre. Hence, the identification of variable stars towards central regions is incomplete because of crowding. The photometric zero-point correction was determined using a sample of constant stars on the reference frame that were cross-correlated with their counterparts in Paper I. To estimate a photometric error, two samples of randomly chosen constant stars common to all images of the time series were selected. Following the same approach as for the reference frame, mean zero-points were calculated from one sample of constant stars for each frame and subsequently used to remove zero-point variations between the various frames. Then, the differences between the corrected magnitudes of all stars of the second sample and the corresponding values from Paper I

\footnotetext{
${ }^{1}$ http://www2.iap.fr/users/alard/package.html
}

were determined. Their standard deviations served as an estimate for the photometric errors. The resulting errors in the $i$-band at a mean luminosity of 19.5 for the various epochs range between 0.085 for NGC 147 and 0. 094 for NGC 185, respectively.

\subsection{Near-infrared data}

$K_{\mathrm{S}}$-band images taken with the NOTCam suffer from distortion that severely increases towards the edge of the frame. Thus, the frames had to be corrected for this effect before carrying out the standard image reduction steps. Gålfalk (2005) constructed a model of the NOTCam WF camera distortion based on his observations of B335. This NOTCam-model ${ }^{2}$ was implemented in a software provided by Gålfalk (2005 written in IDL, which performs additional corrections), which was used for distortion correction of all $K_{\mathrm{s}}$-band images. Subsequently, the usual reduction steps of near-IR imaging were applied to the dithered $K_{\mathrm{s}}$-band images. All frames belonging to one quadrant were aligned and merged to one image to achieve a higher S/N. Pointspread functions fitting photometry was carried out using the DAOPHOT/ALLSTAR photometry package (Stetson \& Harris 1988). The photometric zero-points to calibrate $K_{\mathrm{s}}$ were derived using constant stars in each quadrant of the target galaxy, which were also found in the 2MASS Point Source Catalogue (Cutri et al. 2003). $K_{\mathrm{s}}$-magnitudes of the detected variables in both galaxies are listed in the fifth column of Tables A.1 and A.2, respectively. The corresponding mean photometric uncertainties after the calibration are listed in Table 1 for different bins of magnitude within the range $16<K_{\mathrm{s}}<19$. In Fig. 2 the luminosity function (LF) in $K_{\mathrm{S}}$ of stars detected in NGC 147 and NGC 185 is shown as a black continuous line. The red dashed line represents the distribution of LPVs for which we were able to assign a 

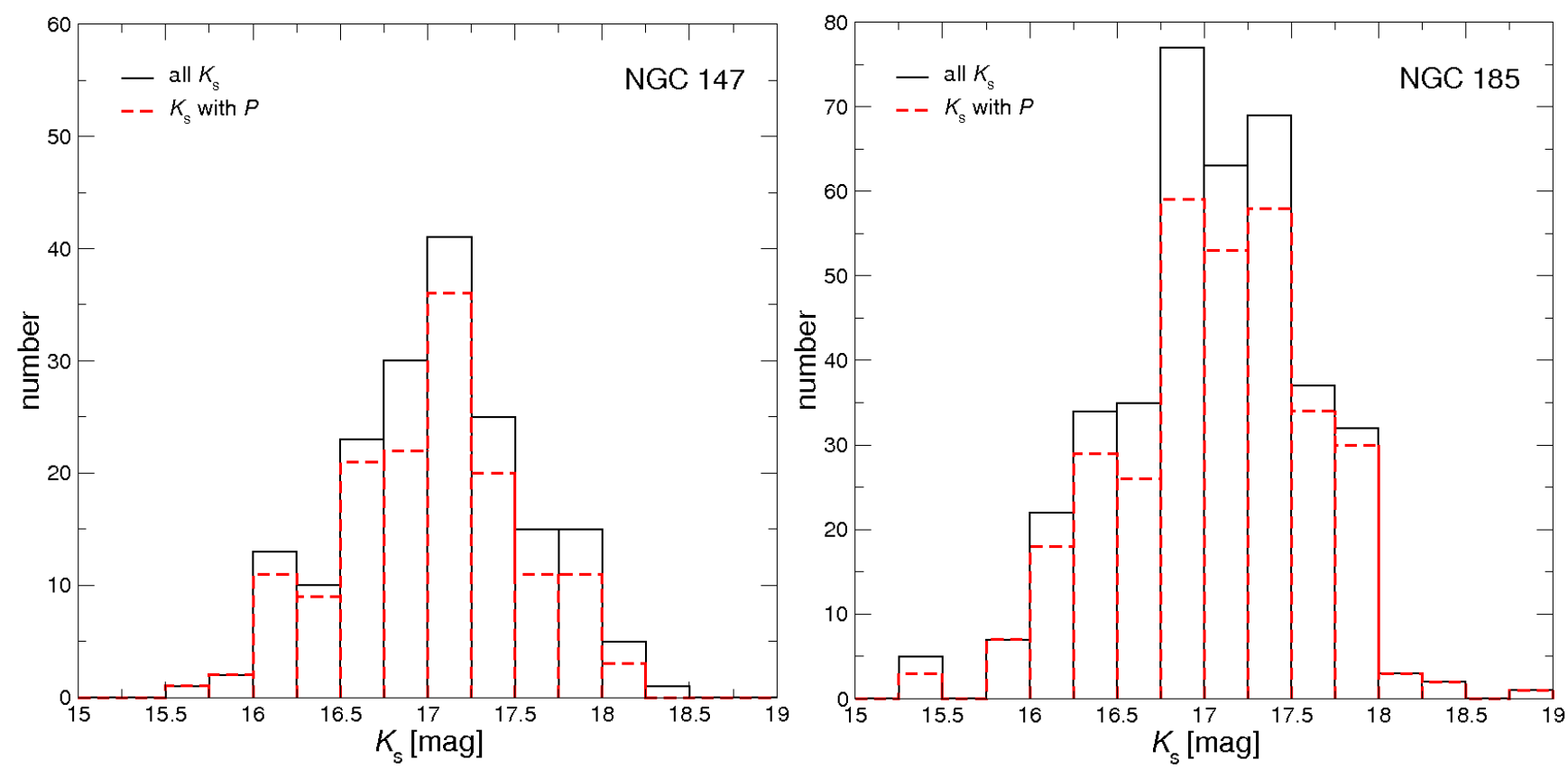

Fig. 2. $K_{\mathrm{s}}$-luminosity functions of objects in NGC 147 and NGC 185. The black line shows the distribution for all detected LPVs with $K_{\mathrm{s}}$-magnitudes available (182 in NGC 147 and 387 in NGC 185). The red dashed line represents LPVs for which it was additionally possible to determine a period (147 in NGC 147 and 323 in NGC 185) as described in Sect. 3.3.

Table 1. Photometric uncertainties for the two galaxies obtained in the $i$ - and $K_{\mathrm{s}}$-filters.

\begin{tabular}{lccc}
\hline \hline$i$ & $e_{\text {phot }}$ & $K_{\mathrm{s}}$ & $e_{\text {phot }}$ \\
\hline$>19$ & 0.03 & $>16$ & 0.09 \\
$19-20$ & 0.04 & $16-17$ & 0.11 \\
$20-21$ & 0.06 & $17-18$ & 0.13 \\
$21-22$ & 0.09 & $18-19$ & 0.18 \\
$<22$ & 0.16 & $<19$ & 0.26 \\
\hline
\end{tabular}

period as well. With the instrument setting mentioned above, the photometry in $K_{\mathrm{s}}$ for both galaxies is probably complete down to $\approx 17 \mathrm{mag}$. At a mean $K$-band luminosity of $17 \mathrm{mag}$, the typical photometric errors for NGC 147 and NGC 185 are 0.15 and 0.16 , respectively.

\subsection{Catalogue of variables}

The $i$-band light curves were searched for periodicities using SIGSPEC $^{3}$ (Reegen 2007). A maximum of two periods was derived from the Fourier analysis if the criterion for significance was fulfilled. The significance (sig) of a period is defined in SIGSPEC as the inverse of the logarithmic scaled false-alarmprobability (FAP) that a discrete Fourier transform amplitude is caused by noise (see Reegen 2007 for details). A spectral significance of 5 therefore corresponds to an inverse FAP of $10^{5}$ or, in other words, the risk of the amplitude being just caused by noise is $1: 10^{5}$. Example light curves showing different types of LPVs from both galaxies together with our best model fit are shown in Fig. 3. The results of the period search are summarised in Tables A.1 and A.2. Beside the periods and corresponding significance of the detected LPVs, the table also lists the mean $i$-magnitudes that were obtained from the light curve.

\footnotetext{
${ }^{3}$ http://www.sigspec.org/
}

The corresponding photometric errors of the mean brightness in the $i$-band after the calibration are listed in Table 1 for different ranges of magnitude. We only used Fourier-amplitudes from the SIGSPEC-output to fit the light curves (see red line in Fig. 3). Additionally, we defined a $\sigma$-amplitude that is twice the statistical standard deviation from the mean brightness of the variable. A purely sinusoidal light curve, for example, with a peak-to-peak-amplitude $A=1$. 0 would result in a corresponding $\sigma$-amplitude of $A_{\sigma}=0.701$ (hereafter $\Delta i$ ). This allows us to have a better understanding of the overall variability of the detected LPVs in both galaxies even for LPVs for which no significant period could be asserted. In addition, this parameter is not sensitive to outliers of the observed light curve mainly caused by dead pixels on the frame or cosmic rays during the integration. Depending on the results of the period search, we were able to assign one, two, or no period to each LPV. For some stars (starting from ID 147V000169 in Table A.1 and ID 185V000420 in Table A.2) it was not possible to detect a significant period, although they clearly are variable. Therefore, we listed their $\sigma$-amplitudes $\Delta i$ to obtain a better impression of their variability.

\subsection{Cross-correlation with photometry from Paper I $(\mathrm{V}, \mathrm{i}, \mathrm{TiO}, \mathrm{CN})$}

In Paper I, single epoch $V i$ photometry was discussed as part of a photometric survey of Local Group galaxies. Furthermore, narrow-band filters (wing-type) were used to derive information on the probable spectral types of the bright red giant stars in these galaxies. To discuss LPVs of NGC 147 and NGC 185 in more detail, a cross-correlation with the results obtained in Paper I was performed using the DENIS software "Cross Color" written by Ch. Alard (see Schuller et al. 2003). This allows us to distinguish C-rich LPVs from other detected variables in our sample and to study their distribution in consecutive diagrams. For approximately $75 \%$ of the identified variables we could 


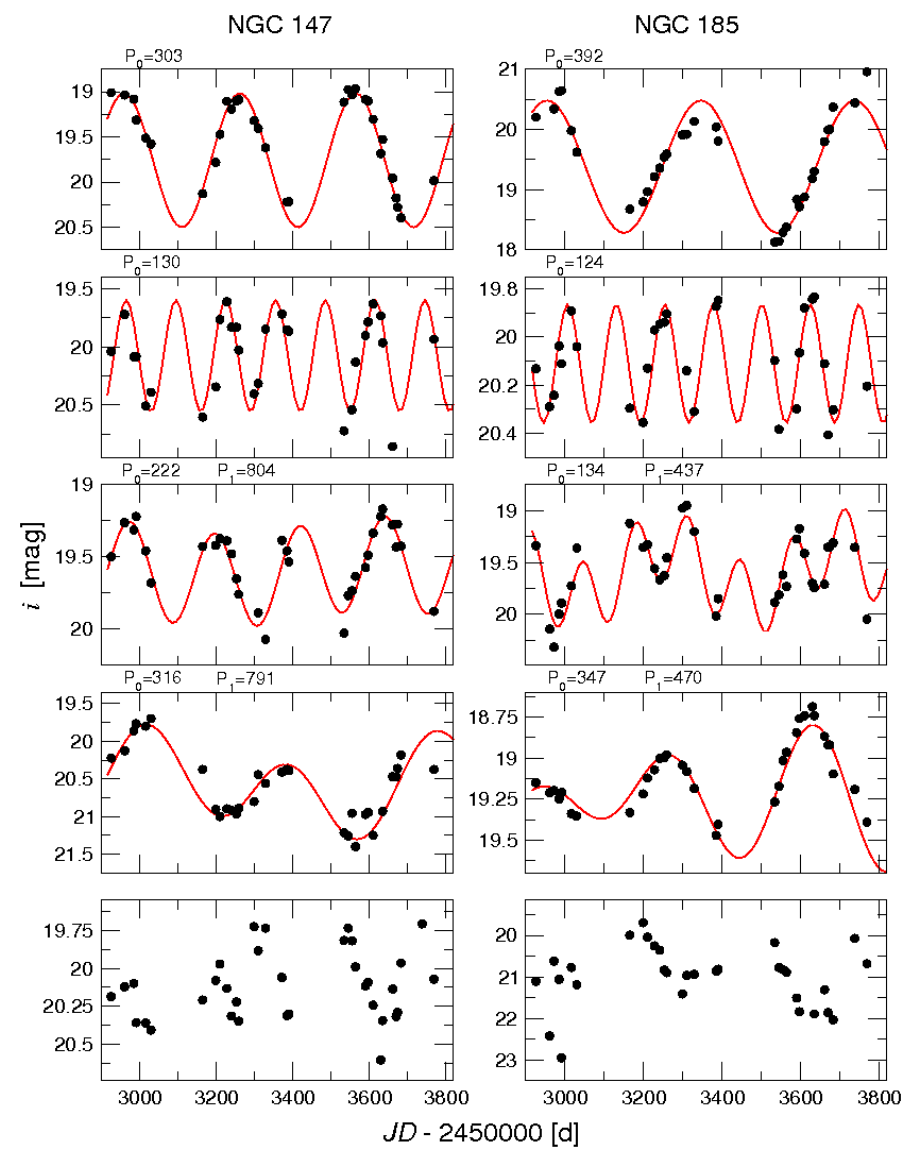

Fig. 3. Example light curves of detected LPVs in each of the target galaxies (NGC 147 on the left and NGC 185 on the right side). The black dots illustrate the observational data, while the red lines display the fitted light curve derived with SIGSPEC (Reegen 2007). Shown are different types of LPVs (mono-periodic variations, two periods, and LPVs with no significant period).

assign counterparts in Paper I. The reason for the incompleteness was threefold. First, some stars were obviously at light minimum and, thus, too weak at the epoch of the observations of Paper I. Second, we had to exclude all variables where the cross-correlation was ambiguous because of crowding. Third, a few stars visible on the frames studied in Paper I had photometric errors that were too large to be included in the final list there.

\section{Results and discussion}

\subsection{Variable stars}

In Tables 2 and 3 we list the number of objects in each of the two galaxies for which certain sets of data are available. We thereby describe the size of several sub-samples grouped according to the information available. For the 163 variables in NGC 147 and the 381 objects in NGC 185 where we have broad-band photometry from Nowotny et al. (2003), the locations in the CMDs are shown in Fig. 4 together with the full sample of Paper I. The LPVs are superimposed as red crosses and those classified as C-rich stars (according to Paper I) are drawn as black circles. Evidently, most of the identified LPVs are located in the upper region of the giant branch where AGB stars, and in particular classical carbon-rich stars, are expected. Approximately two thirds of the variable red giant stars in both galaxies clearly show
Table 2. Summary of detected variables in NGC 147, grouped according to the information available for different sub-samples.

\begin{tabular}{lccccccc}
\hline \hline LPVs & $\times$ & $\times$ & $\times$ & $\times$ & $\times$ & $\times$ & $\times$ \\
Period & & $\times$ & & & $\times$ & $\times$ & $\times$ \\
$V, i$ & & & $\times$ & & & $\times$ & $\times$ \\
TiO, CN & & & & & & $\times$ & $\times$ \\
$K_{\mathrm{S}}$ & & & & $\times$ & $\times$ & & $\times$ \\
\hline objects & 213 & 168 & 163 & 182 & 147 & 122 & 113 \\
\hline
\end{tabular}

Table 3. Same as Table 2 for NGC 185.

\begin{tabular}{lccccccc}
\hline \hline LPVs & $\times$ & $\times$ & $\times$ & $\times$ & $\times$ & $\times$ & $\times$ \\
Period & & $\times$ & & & $\times$ & $\times$ & $\times$ \\
$V, i$ & & & $\times$ & & & $\times$ & $\times$ \\
TiO, CN & & & & & & $\times$ & $\times$ \\
$K_{\mathrm{S}}$ & & & & $\times$ & $\times$ & & $\times$ \\
\hline objects & 513 & 419 & 381 & 387 & 323 & 298 & 229 \\
\hline
\end{tabular}

mono-periodic light variations. Two periods could be assigned to 20 variables of NGC 147, and for 45 LPVs of this system no significant period was found. In NGC 185 we find 38 LPVs exhibiting two periods and 94 LPVs for which no period could be detected. The light curves of LPVs without a significant period show a wide variety of shapes. A part of them definitely shows very long variations that exceed the length of our time series. On the other hand, we also have a short period limit because of the sampling interval of our observations, which amounts to approximately 90 days. We also found objects with irregular light variations, sometimes alternating with phases of comparably constant brightnesses. A selection of different LPV light curves detected in both galaxies is presented in Fig. 3. Monoperiodic cases can be found in the panels of the upper two rows. Examples of LPVs exhibiting two significant periods are plotted in the following two rows, which show a beating phenomenon in the second panel of the fourth row. In the last row of Fig. 3, two cases out of the $\approx 20 \%$ of our sample stars are given for which no significant period could be determined from the observations. However, taking into account their $(V-i), \sigma$-amplitudes and the time scales of their light variations, they can still clearly be classified as LPVs.

Using the narrow-band photometry from Paper I, we can assign a probable atmospheric chemistry to most of the LPVs in our sample, and study the variability characteristics in relation to these defined subgroups. Of special interest are carbon stars, which we assume to be intrinsic post-third-dredge-up objects. Table 4 groups the identified variable stars according to the designated chemistry type. To search for possible correlations between the location of LPVs within the galaxy and their chemistry, we chose one of the CCD images from the $i$-band time series and indicated the detected variables as red circles and C-rich LPVs as blue circles (see Fig. 1). The radial distribution for identified AGB stars in NGC 147 and NGC 185 has been discussed in Paper I. The authors find similar distributions for all AGB stars and for only C-rich AGB stars. If the sample is reduced to detected LPVs in those galaxies, the trend in radial distribution for C-rich LPVs is similar as that for the full sample of 

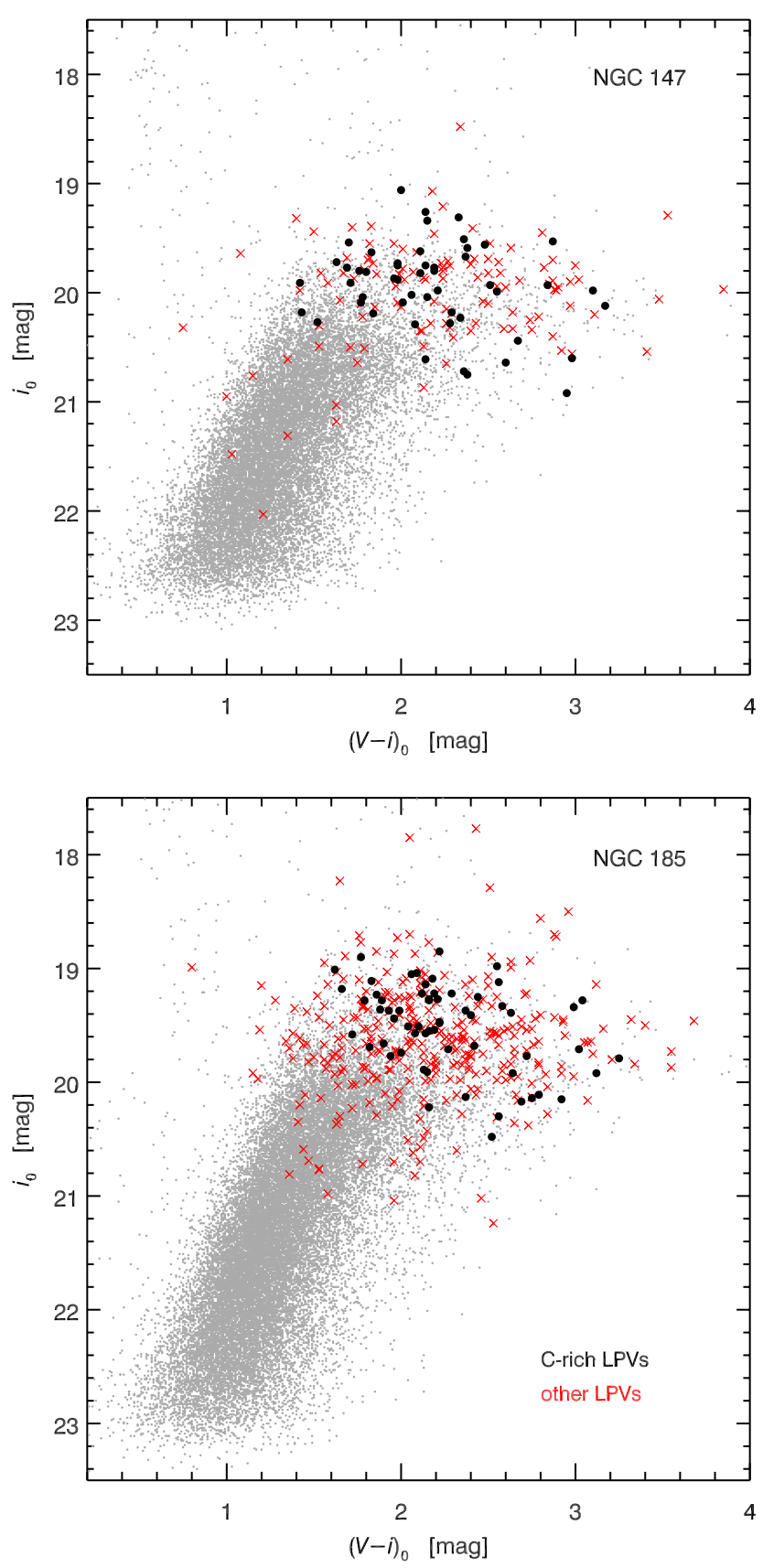

Fig. 4. Colour-magnetude diagrams of stars in NGC 147 (upper panel) and NGC 185 (lower panel) using data from Paper I (grey dots). Overplotted are identified LPVs of the present study (see legend).

Table 4. LPVs identified in the two target galaxies grouped according to their chemical types as derived from the narrow-band photometry of Paper I.

\begin{tabular}{lcc}
\hline \hline & NGC 147 & NGC 185 \\
\hline $\mathrm{C}$ & 51 & 61 \\
$\mathrm{M} / \mathrm{S}$ & 98 & 288 \\
unclass. & 64 & 164 \\
\hline$\Sigma$ & 213 & 513 \\
\hline
\end{tabular}

LPVs. The number ratio of carbon-rich over oxygen-rich LPVs amounts to 0.52 for NGC 147 and 0.21 for NGC 185, respectively. These values fall between the corresponding ratios for the whole population and the ratios when limiting the O-rich sample
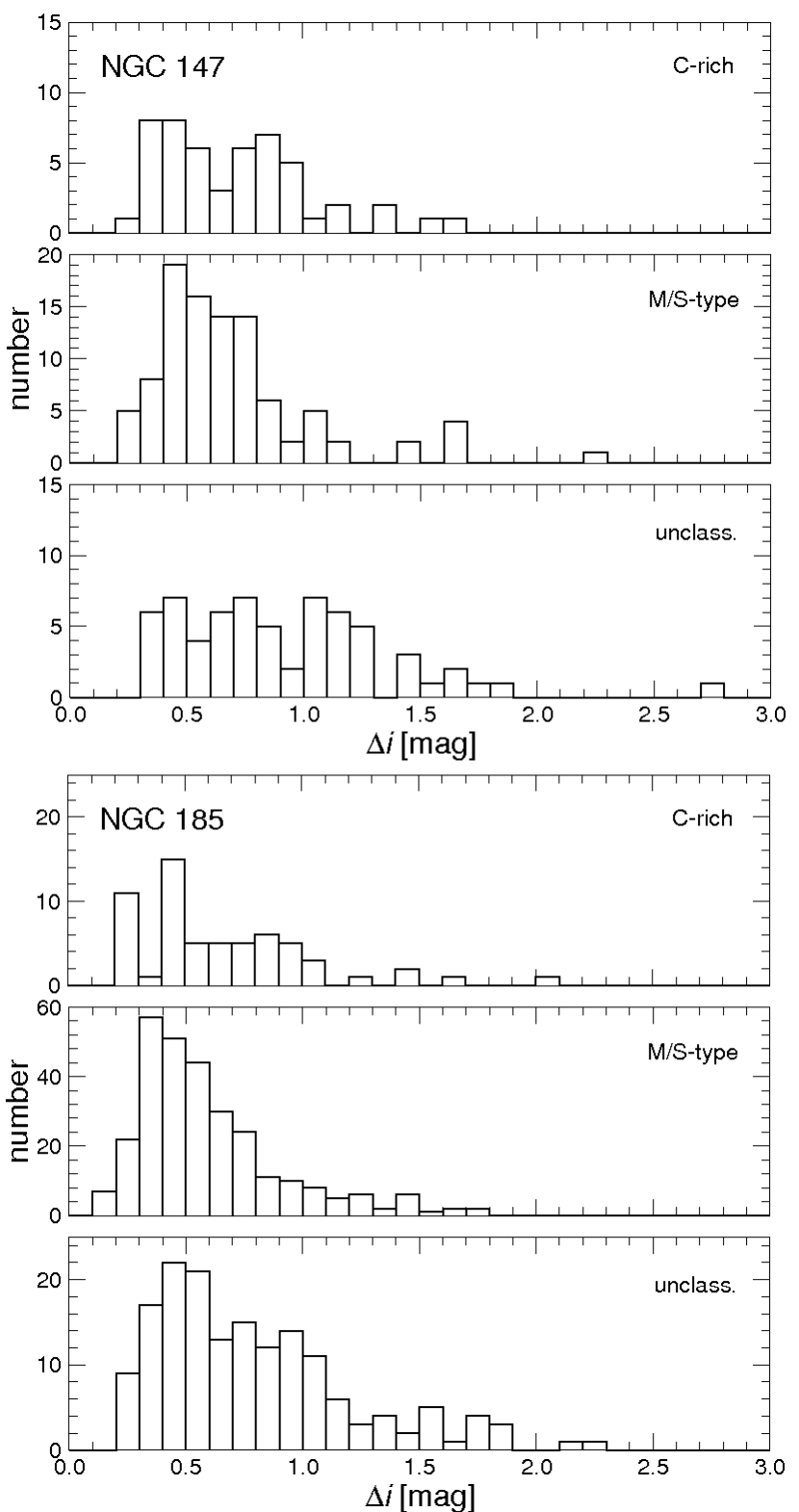

Fig. 5. Histogram of $\sigma$-amplitudes $\Delta i$ of LPVs with different surface chemistry in NGC 147 and NGC 185 . The first panel shows the distribution of C-rich, the second panel displays O-rich LPVs, and the last panel shows LPVs for which no chemical information was available (mainly because of missing narrow-band photometry).

to spectral types M5 and later, as presented in Paper I. Thereby, our sample selected on variability provides a good representation of the AGB population with which the LPV class is typically associated. If we look at the histograms in Fig. 5, we notice that the amplitude distributions of the O-rich objects is dominated by small-amplitude variables. Indeed, the histograms suggest that the peak at 0.35 is not real, but that we are missing stars with the shortest amplitudes owing to the limited sampling rate of our monitoring. In contrast, C-rich stars exhibit a much flatter distribution in these plots. For the sake of completeness, the distribution of LPVs without narrow-band photometry is given in the last panels of Fig. 5 for each of the galaxies. The period distributions of the LPVs in the two galaxies can be seen in the $\Delta i$ vs. $\log P$ diagrams in Fig. 6. Here, we plotted only the first significant period of all detected LPVs. The range of periods covered by 
D. Lorenz et al.: Long-period variables in NGC 147 and NGC 185
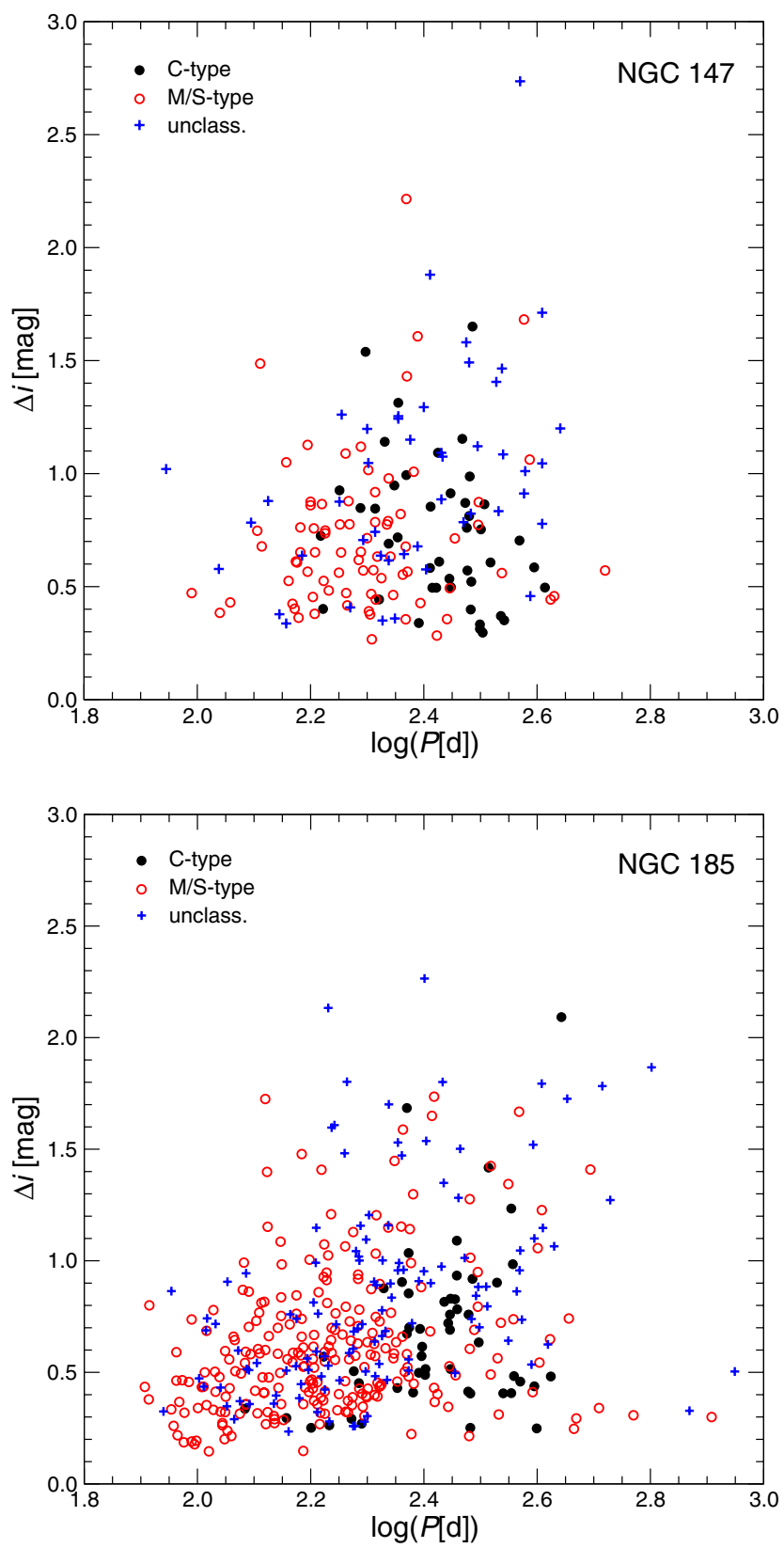

Fig. 6. Photometric amplitudes $\Delta i$ versus periods for LPVs identified in NGC 147 (upper panel) and NGC 185 (lower panel). C-rich objects are indicated by black filled circles, while M/S-type stars are plotted with red open circles, and unclassified LPVs are drawn as blue crosses.

the variables is similar in both systems $\left(\approx 90-600^{\mathrm{d}}\right)$ and a weak tendency of larger amplitudes with increasing period may be visible. Considerably more LPVs with shorter periods were found in NGC 185. In this galaxy, we also found a small group of stars with very long periods and small amplitudes. These are likely candidates for LSPs, but no other significant periodicities were found from our times series. Splitting up the stars according to their chemistry reveals a concentration of C-rich targets around $\log P=2.5$, while the O-rich stars are found predominantly at shorter periods. This behaviour is expected from theory.

\subsection{Period-luminosity relations}

To construct PLDs for both target systems, NGC 147 and NGC 185 , the datasets of the $i$-band time series and the $K_{\mathrm{s}}$-band photometry were combined. This resulted in 182 LPVs with $K_{\mathrm{s}}$-magnitudes and detected periods in NGC 147 and $387 \mathrm{LPVs}$ in NGC 185, respectively, which could be used for the construction of the PLDs. The resulting $K_{\mathrm{s}}-\log P$ diagrams for both galaxies are shown in Fig. 7. Different symbols denote the various classes of LPVs, namely C-rich, O-rich, and unclassified variables according to the narrow-band photometry adopted from Paper I. Furthermore, the amplitude was used to group the variables into four sub-samples indicated by the symbol sizes in Fig. 7. Obviously, most LPVs in both galaxies seem to form a distinct sequence at the very same location in the PLDs as the sequence of fundamental mode pulsators (labeled sequence $C$; cf. Sect. 1) found by various authors for the Magellanic Clouds. For illustration purposes, we overplotted the relations of Ita et al. (2004). To shift their relations according to the difference in distance between the Magellanic Clouds and our galaxies, we adopted the distance moduli determined via the brightness of horizontal branch stars for our target systems by Butler et al. (2005). They derived distance moduli of $(m-M)_{0}=24$. $38 \pm$ 0 . 01 and 24. $09 \pm 0.06 \mathrm{mag}$ for NGC 147 and NGC 185, respectively. For the LMC we adopted the distance modulus obtained by Pietrzyńsky et al. (2009) of $(m-M)_{0}=18$ m.50 \pm 0 m.06. With respect to the atmospheric chemistry, we find O-rich stars along the whole sequence (with a slight thinning in number towards the top), while C-rich stars mainly occupy the upper part of the sequence. This agrees with findings of other studies (e.g., Wood 2000; or Ita et al. 2004). After applying a 3.0 $\sigma$ clipping to exclude stars that are considered not to belong to sequence $\mathrm{C}$, a least-squares fitting was performed to obtain PLRs for this sample of stars. The linear regression $\left(m_{K}=a \log P+b\right)$; black dashed lines in Fig. 7 of this selection resulted in a slope $a$ of -3.55 for NGC 147 and -3.47 for NGC 185 . For the intercepts $b$ of each relation we obtained 25.46 and 25.22 for NGC 147 and NGC 185, respectively. In Table 5 we contrast PLRs derived by different studies with those found here. This comparison of results for different stellar systems is of interest for studying the aspect of the universality of the PLRs of Sequence C stars, which may then serve as an additional tool to measure distances in extragalactic systems. Owing to the limited number of carbon stars in our samples, we did not analyse C-rich and O-rich stars separately. However, for comparison reasons we calculated PLRs for our samples with and without C-rich LPVs. The small differences of the values demonstrate that the results are robust against slight changes of the sample selection. For additional calculations we only consider the results of the complete $3.0 \sigma$ clipping sample. The slopes $a$ found for NGC 147 and NGC 185 are close to those given for the combined samples in other stellar systems.

In Fig. 7 an obvious offset between the shifted LMCPLRs and those derived for NGC 185 can be seen. The numbers in Table 5 suggest that this shift amounts to approximately $0.2-0.4$ (depending on sample selection and regression method). A mild difference in the zero-point $b$ is expected owing to the difference in metallicity (Wood 1990). However, for the lower metallicity of NGC 185 relative to the LMC, a star should be brighter at a given period, making the discrepancy even larger. A simple but not necessarily final explanation for this difference would be an error in the distance modulus of NGC 185. When attempting to bring our observations in line with the LMC relations of Ita et al. (2004), a distance modulus of 24.30 seems to be more appropriate for this galaxy. This value is obtained by 

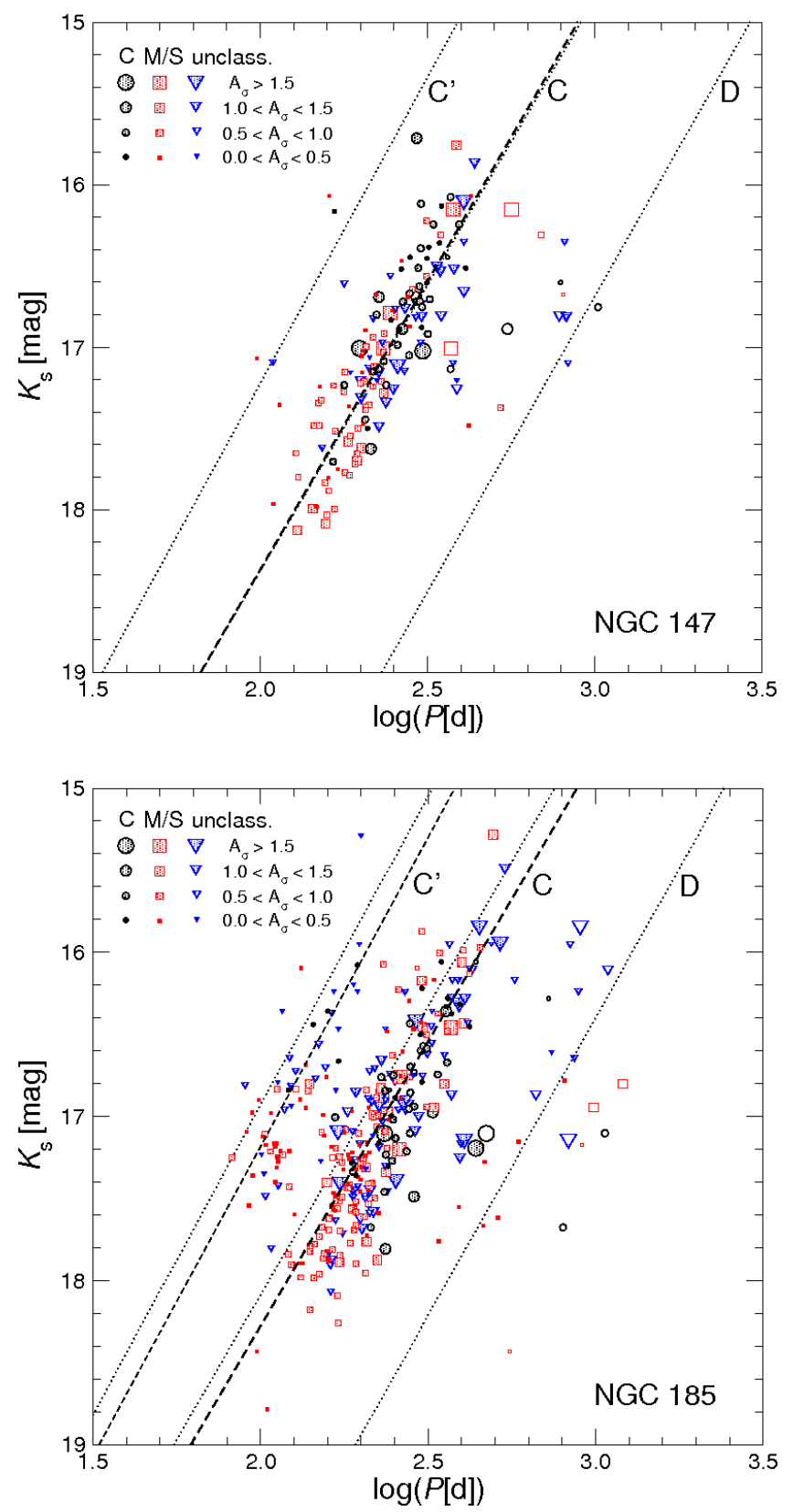

Fig. 7. Period- $K_{\mathrm{s}}$-diagram of NGC 147 (upper panel) and NGC 185 (lower panel). C-type LPVs are plotted as black circles, M/S-type LPVs as red squares and unclassified variable red giant stars as blue triangles. The sizes of the plot symbols are scaled corresponding to the photometric amplitudes $A_{\sigma}$ (see legend). For LPVs with two detected periods, open symbols indicate the second, longer period of both. The black dotted lines $\left(\mathrm{C}^{\prime}, \mathrm{C}, \mathrm{D}\right)$ mark the PLRs found for the LMC by Ita et al. (2004). They were adopted and shifted according to the distance moduli differences between the LMC and our galaxies (see text). The black thick dashed line shows the PLRs derived by using the LPVs identified in this work. The black thin dashed line in the lower panel is plotted for demonstration purpose only (see text).

subtracting the resulting $K_{\mathrm{s}}$-magnitudes derived from the relation of Ita et al. (2004) and from this work at a constant value of $\lg P=2.31$. For NGC 147, the distance modulus from the literature excellently agrees with our data. The zero-point problem for NGC 185 needs further exploration. However, if the reason for the offset PLRs is indeed an error in the distance modulus,
Table 5. Comparison of our $P$ - $L$ relations with literature values for different stellar systems

\begin{tabular}{lcccc}
\hline \hline \multicolumn{5}{c}{$M_{K_{\mathrm{s}}}=a \log P+b$} \\
& $a$ & $b$ & $(m-M)$ & Refs. \\
\hline Galactic & $-3.56 \pm 0.17$ & $1.14 \pm 0.42$ & & 1 \\
LMC all & $-3.52 \pm 0.03$ & $1.04 \pm 0.08$ & 18.50 & 2 \\
LMC & & & & \\
all & $-3.34 \pm 0.02$ & $0.40 \pm 0.05$ & 18.50 & 3 \\
O-rich & $-3.31 \pm 0.04$ & $0.47 \pm 0.09$ & & 3 \\
C-rich & $-3.16 \pm 0.04$ & $-0.10 \pm 0.11$ & & 3 \\
LMC & & & & \\
all & $-3.57 \pm 0.16$ & $1.20 \pm 0.39$ & 18.50 & 4 \\
O-rich & $-3.47 \pm 0.19$ & $0.98 \pm 0.45$ & & 4 \\
C-rich & $-3.30 \pm 0.40$ & $0.48 \pm 0.98$ & & 4 \\
Cen A & $-3.37 \pm 0.11$ & $0.80 \pm 0.29$ & 27.87 & 5 \\
\hline NGC 147 & & & & \\
all & $-3.55 \pm 0.15$ & $1.08 \pm 0.36$ & 24.38 & 6 \\
O-rich & $-3.81 \pm 0.25$ & $1.68 \pm 0.58$ & 24.38 & 6 \\
NGC 185 & & & & \\
all & $-3.47 \pm 0.11$ & $1.13 \pm 0.27$ & 24.09 & 6 \\
O-rich & $-3.72 \pm 0.16$ & $1.68 \pm 0.38$ & 24.09 & 6 \\
\hline
\end{tabular}

Notes. All relations were shifted to an absolute scale $M_{K}$ using the distance moduli given in the text.

References. (1) Groenewegen \& Whitelock (1996); (2) Ita et al. (2004); (3) Riebel et al. (2010); (4) Feast et al. (1989); (5) Rejkuba (2004); (6) this work.

this would be the first correction of a distance to a galaxy based on PLRs of LPVs.

For NGC 185 there are indications of another parallel sequence of LPVs shifted towards shorter periods (sequence $\mathrm{C}^{\prime}$ ). On average, stars on this sequence exhibit smaller amplitudes than objects on sequence $C$ (see lower panel of Fig. 7). A similar PLR was found in the LMC (e.g. Ita et al. 2004; Fraser et al. 2008) and is associated with first overtone pulsation. The smaller light amplitude of this group identified in our sample agrees with this interpretation. Note that the trend for the zero point of sequence $\mathrm{C}$ is also visible for sequence $\mathrm{C}^{\prime}$ but the number of detected LPVs populating sequence $\mathrm{C}^{\prime}$ in NGC 185 prohibits another linear regression. For demonstration purpose only, we plotted sequence $\mathrm{C}^{\prime}$ of Ita et al. (2004) and shifted it according to the difference in distance obtained for sequence $\mathrm{C}$. This line is drawn as thin dashed line in Fig. 7. A handful of targets in NGC 147 may also be located on this sequence. Variables corresponding to higher overtone pulsation were not accessible to our study owing to the sampling rate limitations.

From studies in various stellar systems it is known that the LSPs, visible in a significant fraction of all LPVs, form another sequence to the right of sequence $\mathrm{C}$ in the PLD. This sequence $\mathrm{D}$, taken from Ita et al. (2004), is overplotted in both panels of Fig. 7 as well. Evidently, we found objects in our variability study for which LSPs derived from the light curve seem to cluster around this sequence D. In these cases, the primary period is typically located close to the fundamental mode pulsation sequence. Note that a detailed determination of these LSPs in our sample is hampered by the limited length of our time series. As described in Sect. 1, the interpretation of this kind of variability is still a matter of debate. Picking up the results of Wood \& Nicholls (2009) that LSP-stars show a significant mid-infrared excess (circumstellar dust), the corresponding targets from our monitoring could be expected to be promising candidates for detecting signatures of circumstellar material. 


\subsection{A hidden link to the star-formation history?}

The similar datasets for the two galaxies with comparable properties (cf. Sect. 1) encouraged us to make a detailed comparison of the derived PLDs (Fig. 7). Besides the different number of LPVs detected (Table 4), which is likely related to the different masses of the systems, the most obvious distinction is the fraction of luminous stars found along sequence $C^{\prime}$. For NGC 185 roughly $10 \%$ of all LPVs can be attributed to this sequence for first overtone pulsation, while only less than $3 \%$ of these stars were detected in NGC 147. This raises the question whether some fundamental differences between the two galaxies are mirrored in the recognised discrepancy. A possible interpretation of the lack of stars on sequence $\mathrm{C}^{\prime}$ in NGC 147 could involve a difference in the mass distribution of these objects. Linear pulsation models (Fox \& Wood 1982) as well as observational results from LPVs in stellar clusters (Lebzelter \& Wood 2005, 2007) suggest an evolutionary path of an AGB star through the PLD starting on an overtone sequence and later, at higher luminosities, switching to the fundamental mode sequence. Because there are variables in NGC 185 on sequence $C^{\prime}$ with the same luminosity as the bulk of the stars along sequence $\mathrm{C}$, this points towards a higher mass of these stars.

How can this difference in the mass distribution be explained? The most obvious approach would be to assume that the two galaxies differ in their SFH. Indications for this were found in previous studies and become apparent, for example, in the SFH diagrams of Mateo (1998, Fig. 8). Hints for a recent star-formation episode can be found for NGC 185, namely a small population of younger stars concentrated in the central regions, a significant amount of interstellar gas, and prominent dust patches (Sect. 1 and Paper I). On the other hand, NGC 147 seems to be free of dust and gas, and there are no indications for a population younger than 1 Gyr (Han et al. 1997, Paper I). Another hint in this direction may be the possible detection of a small shift in the light amplitude distribution of the LPVs (Fig. 5) in the two systems. A younger system is expected to contain more stars with higher masses and, thus, smaller amplitudes (Lebzelter \& Wood 2007) compared to older systems.

Theory predicts a linear trend between the mean metallicity and the ratio of C-type to M-type stars of a galaxy, which has been confirmed by observations (Iben \& Renzini 1983; Mouhcine \& Lançon 2003). For systems with lower mean metallicities the production of C-type stars is favoured. According to the values obtained in Paper I, NGC 185 is considered as the more metal-poor galaxy. However, the number of C-type stars is approximately the same in both systems, which leads to a much lower C/M ratio for NGC 185 than for NGC 147 (0.21 and 0.52 , respectively). Note that one has to be careful with the interpretation of this result because Battinelli \& Demers (2005) clearly demonstrated how severely the $\mathrm{C} / \mathrm{M}$ ratio depends on the selection criterion (see their Figs. 3 and 4). In addition, the mean metallicity of a galaxy is an elusive parameter because galaxies consist of multiple populations with a mixture of ages and metallicities. The small separation (within the uncertainties) of our target galaxies in Fig. 3 of Battinelli \& Demers (2005) does not allow us to draw any conclusions.

The SFHs of the two galaxies should be explored in more detail to arrive at a final interpretation of this question. Because both galaxies, NGC 147 and NGC 185, are comparable in properties (distance, luminosity, C-star content) but differ in their SFHs, they appear to be ideal candidates to shed light on this challenging topic.

\section{Conclusions}

A photometric monitoring in the $i$-band of the two Local Group dwarf galaxies NGC 147 and NGC 185 led to the identification of 213 and 513 LPVs, respectively. Narrow-band photometry adopted from Paper I allowed us to investigate the number of $\mathrm{C}$-rich and O-rich stars among this variability class. Thus, our study is one of the few (e.g., Groenewegen 2004) that uses a more elaborated chemistry separation than the often used broadband colour criterion (e.g., $(J-K)>1.4)$. Because the attribute of long-period variability is more significant than a pure brightness limit to select the AGB stars among all late-type giants of a population, the ratio of C-/M-type LPVs is a more reliable measure of the corresponding ratio on the AGB. From our study we determine a value of 0.52 for NGC 147 and 0.21 for NGC 185 . Our substantial sample of LPVs allowed us to investigate the corresponding PLRs in the $K_{\mathrm{s}}-\log P$-plane as well. Most variables in both galaxies are located along sequence $C$, where fundamental mode pulsators are theoretically expected. A linear regression $\left(m_{K}=a \log P+b\right)$ was fitted to the data of these stars. The resulting fit parameters agree well with the corresponding values found for the LMC in the literature (e.g., Ita et al. 2004). This allows us to speculate further about the universality of the P-L-relation of LPVs. However, we noticed a discrepancy in $b$ for NGC 185, which may point to an error in the previously derived distance modulus of this galaxy. This would be the first correction of a distance to a galaxy based on PLRs of LPVs. The most significant difference between the PLDs of the two target systems is the presence of a group of first overtone pulsators in NGC 185, which is almost completely missing in NGC 147. We speculate that this effect may be explained by a difference in the star-formation history and, accordingly, a different mass distribution on the AGB. According to our sampling interval, periods shorter than 90 days could not be determined. We intend to extend our monitoring by obtaining more observations at a higher sampling rate, which will allow the detection of these periods. Moreover, these additional data points would increase the chance to properly describe the LSPs that are likely present in a significant fraction of our LPV sample.

Acknowledgements. The data presented here have been taken using ALFOSC, which is owned by the Instituto de Astrofisica de Andalucia (IAA) and operated at the Nordic Optical Telescope under agreement between IAA and the NBIfAFG of the Astronomical Observatory of Copenhagen. This work was supported by the Fonds zur Förderung der Wissenschaftlichen Forschung (FWF) under project numbers P18939-N16, P20046-N16 and P21988-N16. This research has made use of the NASA/IPAC Extragalactic Database (NED) which is operated by the Jet Propulsion Laboratory, California Institute of Technology, under contract with the National Aeronautics and Space Administration.

\section{References}

Alard, C. 2000, A\&AS, 144, 363

Battinelli, P., \& Demers S. 2004a, A\&A, 417, 479

Battinelli, P., \& Demers S. 2004b, A\&A, 418, 33

Battinelli, P., \& Demers S. 2005, A\&A, 434, 657

Beaulieu, J. P., Carey, S., Ribas, I., \& Tinetti, G. 2008, ApJ, 677, 1343

Butler, D. J., \& Martinez-Delgado D. 2005, AJ, 129, 2217

Cioni, M.-R. L., Marquette, J. B., Loup, C., et al. 2000, A\&A, 377, 945

Corradi, R. L. M., Magrini, L., Greimel, R., et al. 2005, A\&A, 431, 555

Cutri, R. M., Skrutskie, M. F., Van Dyk, S., et al. 2003, Expl. Supplement to the 2MASS All Sky Data Release, http://www.ipac.caltech.edu/2mass/ releases/allsky/doc/explsup.html

Dolphin, A. E. 2000, ApJ, 531, 804

Feast, M. W., Glass, I. S., Whitelock, P. A., et al. 1989, MNRAS, 241, 375 Fox, M. W., \& Wood, P. R. 1982, ApJ, 259, 198

Fraser, O. J., Hawley, S. L., Cook, K. H., \& Keller, S. C. 2005, AJ, 129, 768

Fraser, O. J., Hawley, S. L., Cook, \& K. H. 2008, AJ, 136, 1242 
Girardi, L., Bertelli, G., Bressan, et al. 2002, A\&A, 391, 195 Gålfalk, M. 2005, NOT Annual report 2004, p18

Geha, M., van der Marel, R. P., Guhathakurta, P., et al. 2010, ApJ, 711, 361 Groenewegen, M. A. T. 2004, A\&A, 425, 595

Groenewegen, M. A. T. 2005, ASP Conf. Ser. [arXiv: astro-ph/0506381] Groenewegen, M. A. T. 2007, ASP Conf. Ser., 378, 433

Groenewegen, M. A. T., \& Whitelock P. A. 1996, MNRAS, 281, 1347 Han, M., Hoessel, J. G., Gallagher III, J. S., et al. 1997, AJ, 113, 1001 Iben, Jr., I., \& Renzini, A. 1983, ARA\&A, 21, 271

Ita, Y., Tanabé, T., Matsunaga, N., et al. 2004, MNRAS, 347, 720

Kang, A., Sohn, Y.-J., Rhee, J., et al. 2005, A\&A, 437, 61

Kiss, L. L., \& Bedding, T. R. 2003, MNRAS, 343, L79

Lebzelter, T., \& Wood, P. R. 2005, A\&A, 441, 1117

Lebzelter, T., \& Wood, P. R. 2007, A\&A, 475, 643

Lebzelter, T., Schultheis, M., \& Melchior, A. L. 2002, A\&A, 393, 573

Lee, M. G., Freedman, W. L., \& Madore, B. F. 1993, ApJ, 417, 553

Martínez-Delgado, D., \& Aparicio A. 1998, AJ, 115, 1462

Martínez-Delgado, D., Aparicio A., \& Gallart, C. 1999, AJ, 118, 2229

Mateo, M. 1998, ARA\&A, 36, 435

Mouhcine, M., \& Lançon, A. 2003, MNRAS, 338, 572

Nicholls, C. P., Wood, P. R., Cioni M.-R. L., et al. 2009, MNRAS, 399, 2063
Nicholls, C. P., Wood, P. R. \& Cioni M.-R. L. 2010, MNRAS, 405, 1770

Nowotny, W., Kerschbaum, F., Olofsson, H., et al. 2003, A\&A, 403, 93 (Paper I) Nowotny, W., Höfner, S., \& Aringer, B. 2010, A\&A, 514, A35

Pietrzyńsky, G., Thompson, I. B., Graczyk, D., et al. 2009, AJ, 697, 862

Reegen, P. 2007, A\&A, 467, 1353

Rejkuba, M. 2004, A\&A, 413, 903

Rejkuba, M., Minniti, D., \& Silva, D. R. 2003, A\&A, 406, 75

Riebel, D., Meixner, M., Fraser, O., et al. 2010, ApJ, 723, 1195

Sage, L. J., Welch, G. A., \& Mitchell, G. F. 1998, AJ, 507, 726

Schuller, P., Ganesh, S., Messineo, M., et al. 2003, A\&A, 403, 955

Sohn, Y.-J., Kang, A., Rhee, J., et al. 2006, A\&A, 445, 69

Soszyński, I., Udalski, A., Kubiak, M., et al. 2004a, Acta Astron., 54, 129

Soszyński, I., Udalski, A., Kubiak, M., et al. 2004b, Acta Astron., 54, 347

Stetson, P. B., \& Harris, W. E. 1988, AJ, 96, 909

van den Bergh S. 1998, AJ, 116, 1688

Wood, P. R. 1990, in From Miras to Planetary Nebulae, ed. M. O. Mennessier, \& A. Omont (Gif-sur-Yvette: Éditions Frontières), 67

Wood, P. R. 1999, IAU Symp., 191, 151

Wood, P. R. 2000, PASA, 17, 18

Wood, P. R., \& Nicholls, C. P. 2009, ApJ, 707, 573

Young, L. M., \& Lo, K. 1997, AJ, 476, 127

Pages 11 to 23 are available in the electronic edition of the journal at http://www . aanda. org 
Appendix A:

Table A.1. Period search results of NGC 147.

\begin{tabular}{|c|c|c|c|c|c|c|c|c|c|c|c|}
\hline ID & $\begin{array}{c}\text { RAJ2000 } \\
\text { h:m:s }\end{array}$ & $\begin{array}{c}\text { DEJ2000 } \\
\text { d:m:s }\end{array}$ & $\begin{array}{c}i \\
{[\mathrm{mag}]}\end{array}$ & $\begin{array}{c}K_{\mathrm{s}} \\
{[\mathrm{mag}]}\end{array}$ & $\begin{array}{l}P_{0} \\
{[\mathrm{~d}]}\end{array}$ & $\begin{array}{c}\Delta i \\
{[\mathrm{mag}]}\end{array}$ & $\operatorname{sig}_{0}$ & $\begin{array}{l}P_{1} \\
\text { [d] }\end{array}$ & $s i g_{1}$ & type & ID $_{\text {Paper I }}$ \\
\hline $147 \mathrm{~V} 000001$ & 003330.99 & 483218.07 & 20.06 & - & 241 & 1.01 & 2.1 & - & - & $\mathrm{S}$ & 00971672 \\
\hline $147 \mathrm{~V} 000002$ & 03328.57 & 482933.85 & 20.20 & - & 258 & 0.85 & 4.6 & - & - & $\mathrm{C}$ & 02190795 \\
\hline $147 \mathrm{~V} 000003$ & 003327.59 & 482948.00 & 20.47 & - & 206 & 0.74 & 5.3 & - & - & $\mathrm{u}$ & - \\
\hline $147 \mathrm{~V} 000004$ & 003327.66 & 483033.00 & 19.70 & 16.66 & 300 & 0.57 & 5.7 & - & - & $\mathrm{C}$ & 02691110 \\
\hline $147 \mathrm{~V} 000005$ & 003327.19 & 482929.93 & 19.91 & - & 196 & 0.71 & 5.6 & - & - & $\mathrm{u}$ & 02920773 \\
\hline $147 \mathrm{~V} 000006$ & 003326.68 & 482916.68 & 19.88 & 17.30 & 211 & 0.54 & 4.6 & - & - & M & 03180702 \\
\hline $147 \mathrm{~V} 000007$ & 003326.43 & 483038.81 & 20.60 & 16.55 & 314 & 0.87 & 4.3 & - & - & $\mathrm{S}$ & 03351141 \\
\hline $147 \mathrm{~V} 000008$ & 003325.15 & 483250.78 & 20.12 & 17.24 & 231 & 0.55 & 4.5 & - & - & M & 04081845 \\
\hline $147 \mathrm{~V} 000009$ & 003324.73 & 482934.89 & 20.36 & - & 158 & 0.86 & 5.3 & - & - & $\mathrm{S}$ & 04220799 \\
\hline $147 \mathrm{~V} 000010$ & 003324.32 & 482728.13 & 20.04 & - & 168 & 0.74 & 3.6 & - & - & M & 04390122 \\
\hline $147 \mathrm{~V} 000011$ & 003323.95 & 483247.62 & 19.70 & 17.39 & 206 & 0.79 & 5.8 & - & - & M & 1828 \\
\hline 000012 & 23.63 & 483 & 20.16 & 17.31 & 234 & 1.43 & 5.5 & - & - & M & 1264 \\
\hline 00013 & & 483 & & 16.6 & 299 & 0.76 & 5.2 & - & - & $\mathrm{C}$ & 05081865 \\
\hline 00014 & 003321.58 & 482843.51 & 19.92 & 17.42 & 203 & 0.47 & 5.8 & - & - & M & 05870523 \\
\hline 00015 & 003320.96 & 4830 & 19.89 & 16.77 & 245 & 1.61 & 5.4 & - & - & M & 06251212 \\
\hline 00016 & 3320.82 & 4832 & 20.11 & 17.30 & 178 & 0.56 & 4.3 & - & - & M & 1618 \\
\hline $147 \mathrm{~V} 000017$ & 003320.57 & 483056.92 & 19.84 & 16.15 & 167 & 0.40 & 4.6 & - & - & $\mathrm{C}$ & 06461236 \\
\hline $147 \mathrm{~V} 000018$ & 003320.53 & 483308.16 & 19.71 & 17.51 & 150 & 0.61 & 4.5 & - & - & M & 06531937 \\
\hline $147 \mathrm{~V} 000019$ & 003320.30 & 483028.00 & 19.87 & 16.98 & 233 & 0.68 & 6.1 & - & - & $\mathrm{S}$ & 06591081 \\
\hline 000020 & 003320.30 & 483114.54 & 19.79 & 16.27 & 329 & 0.61 & 6.6 & - & - & $\mathrm{C}$ & 06601330 \\
\hline 00021 & 003320.13 & 483107.14 & 20.12 & 16.69 & 280 & 0.9 & 5.6 & - & - & $\mathrm{C}$ & 06691290 \\
\hline 00022 & 003320.13 & 483232.56 & 19.68 & 16.25 & 313 & 0.7 & 6.2 & - & - & M & 06731747 \\
\hline 00023 & 3319.30 & 48310 & 9.77 & 17.26 & 166 & 0.8 & 5.5 & - & - & $\mathrm{S}$ & 31282 \\
\hline 00024 & 3318.81 & 48292 & 19.89 & 17.10 & 218 & 0.69 & 6.4 & - & - & $\mathrm{C}$ & 07350767 \\
\hline 00025 & 00 & 4829 & .86 & 16.30 & 343 & 0.3 & 4.7 & - & - & $\mathrm{C}$ & 0763 \\
\hline 0026 & .08 & $48 ?$ & & - & & 0. & 4. & - & - & $\mathrm{S}$ & \\
\hline 027 & 00 & $48 ?$ & & 15.74 & & & & - & - & $\mathrm{C}$ & \\
\hline 147 & .65 & 483 & .85 & 16.64 & 276 & 0.3 & & - & - & M & 051 \\
\hline 0029 & 00331 & 483 & 21.18 & 17.05 & 306 & 1.6 & 5. & - & - & $\mathrm{C}$ & 352 \\
\hline 00030 & 3317.03 & 482 & 20.13 & 16.59 & 285 & 0.7 & 6.1 & - & - & $\mathrm{S}$ & 0586 \\
\hline $147 \mathrm{~V} 000031$ & 003316.75 & 483126.32 & 21.23 & 16.52 & 337 & 1.4 & 3.7 & - & - & $\mathrm{u}$ & 08491392 \\
\hline $147 \mathrm{~V} 000032$ & 003316.53 & 483158.91 & 20.38 & 16.42 & 302 & 0.81 & 5.2 & - & - & $\mathrm{C}$ & 08621566 \\
\hline 00033 & 003316.28 & 483211.16 & 9.83 & 16.09 & 427 & 0.4 & 4.6 & - & - & $\mathrm{S}$ & 08761631 \\
\hline 0034 & 003316.08 & 483303.13 & .98 & 17.08 & 212 & 0.3 & 3.7 & - & - & $\mathrm{u}$ & 08891909 \\
\hline 0035 & 331 & 4832 & 20.13 & 16.96 & 217 & 0.7 & 5. & - & - & M & 1662 \\
\hline 0036 & 331 & 4832 & 9.85 & 16.90 & 280 & 0.4 & 4.9 & - & - & $\mathrm{S}$ & 1829 \\
\hline 0037 & .43 & 482 & 0.09 & 17. & 21 & 0. & & - & - & M & 8810 \\
\hline 0038 & & .25 & 19.93 & 17.34 & 114 & 0.4 & 5.2 & - & - & M & 0209 \\
\hline 00039 & & 13 & 44 & 6.54 & 297 & 0.8 & 5.7 & - & - & $\mathrm{C}$ & 877 \\
\hline & & 97 & & 16.50 & 11 & 0. & & - & - & $\mathrm{C}$ & 838 \\
\hline 147 & & 3.77 & .96 & - & 52 & & & - & - & M & 1003 \\
\hline 147 & 3.31 & 6.14 & .57 & 17.26 & 151 & 0.3 & 5.5 & - & - & M & 10270801 \\
\hline 00043 & 313.20 & 48293 & 8.96 & 6.45 & 265 & 0.2 & 5.4 & - & - & M & 10320776 \\
\hline $147 \mathrm{~V} 000044$ & 3313.21 & 483245.73 & 9.82 & 17.08 & 201 & 0.3 & 4.1 & - & - & $\mathrm{S}$ & 10401815 \\
\hline $147 \mathrm{~V} 000045$ & 3313.09 & 483030.14 & 19.60 & 16.90 & 206 & 0.44 & 5.3 & - & - & M & 10401090 \\
\hline $147 \mathrm{~V} 000046$ & 3312.83 & 483121.27 & 19.66 & 16.59 & 245 & 0.68 & 5.8 & - & - & $\mathrm{u}$ & 10561364 \\
\hline $147 \mathrm{~V} 000047$ & 003312.69 & 482826.45 & 20.45 & 17.60 & 200 & 1.0 & 5.7 & - & - & M & 10560428 \\
\hline $147 \mathrm{~V} 000048$ & 003312.72 & 48295 & 9.11 & 16.05 & 161 & 0.3 & 6.4 & - & - & M & 10580915 \\
\hline $147 \mathrm{~V} 000049$ & $0033 \quad 12.14$ & 483102.73 & 20.10 & 17.61 & 183 & 1.0 & 5.7 & - & - & M & 10921264 \\
\hline $147 \mathrm{~V} 000050$ & $0033 \quad 12.14$ & 483209.62 & 21.03 & 18.16 & 129 & 1.4 & 4.9 & - & - & M & 10951622 \\
\hline $147 \mathrm{~V} 000051$ & 003311.67 & 482938.35 & 20.20 & 17.14 & 229 & 0.8 & 4.7 & - & - & M & 11130813 \\
\hline $147 \mathrm{~V} 000052$ & 003 & 482944.64 & 20.09 & 17.77 & 171 & 0.4 & 5.8 & - & - & $\mathrm{S}$ & 11330846 \\
\hline $147 \mathrm{~V} 000053$ & 003311.07 & 482847.60 & 20.10 & 17.68 & 194 & 0.6 & 5.1 & - & - & M & 11430541 \\
\hline $147 \mathrm{~V} 000054$ & & 482854.96 & & 17.01 & 203 & 0.27 & 4.5 & - & - & $\mathrm{S}$ & 11530580 \\
\hline & & & & & 130 & 0.6 & & - & - & $\mathrm{S}$ & 11750488 \\
\hline $147 \mathrm{~V} 000056$ & 003310.42 & 482745.13 & & 16.06 & 370 & 0.7 & 5.5 & - & - & $\mathrm{C}$ & 11750206 \\
\hline $147 \mathrm{~V} 000057$ & 003310.49 & 483314.27 & & 17.1 & 216 & 0.7 & 5.8 & - & - & M & 11851968 \\
\hline $147 \mathrm{~V} 000058$ & 003310.09 & 483312.70 & & 16.55 & 264 & 0.5 & 5.6 & - & - & $\mathrm{C}$ & 12061959 \\
\hline $147 \mathrm{~V} 000059$ & 003309.02 & 482935.53 & 20.22 & 17.90 & 161 & 0.76 & 5.0 & - & - & S & 12540797 \\
\hline $147 \mathrm{~V} 000060$ & & 483233.29 & & 17.38 & 14 & 0.6 & 5.4 & - & - & S & 12631748 \\
\hline & & 482936.66 & 19.65 & 16.4 & 316 & 0.3 & 4.2 & - & - & $\mathrm{C}$ & 12810802 \\
\hline $147 \mathrm{~V} 000062$ & 308.43 & 482933.56 & 20.65 & 16.9 & 19 & 1.5 & 4.9 & - & - & $\mathrm{C}$ & 12850786 \\
\hline $147 \mathrm{~V} 000063$ & 003308.46 & 483017.49 & 20.06 & 17.68 & 195 & 1.1 & 5.4 & - & - & $\mathrm{S}$ & 12851021 \\
\hline $147 \mathrm{~V} 000064$ & 003307.85 & 482848.17 & 20.12 & 18.00 & 147 & 0.42 & 5.6 & - & - & M & 13130543 \\
\hline
\end{tabular}


Table A.1. continued.

\begin{tabular}{|c|c|c|c|c|c|c|c|c|c|c|c|}
\hline ID & $\begin{array}{c}\text { RAJ2000 } \\
\text { h:m:s }\end{array}$ & $\begin{array}{l}\text { DEJ2000 } \\
\text { d:m:s }\end{array}$ & $\begin{array}{c}i \\
{[\mathrm{mag}]}\end{array}$ & $\begin{array}{c}K_{\mathrm{s}} \\
{[\mathrm{mag}]}\end{array}$ & $\begin{array}{l}P_{0} \\
{[\mathrm{~d}]}\end{array}$ & $\begin{array}{c}\Delta i \\
{[\mathrm{mag}]}\end{array}$ & $\operatorname{sig}_{0}$ & $\begin{array}{l}P_{1} \\
{[\mathrm{~d}]}\end{array}$ & $\operatorname{sig}_{1}$ & type & ID $D_{\text {Paper I }}$ \\
\hline 147V000065 & 003307.53 & 483022.15 & 20.04 & 17.67 & 128 & 0.75 & 5.7 & - & - & $\bar{M}$ & 13341046 \\
\hline $147 \mathrm{~V} 000066$ & 003307.14 & 482949.17 & 20.01 & 17.23 & 205 & 0.57 & 5.5 & - & - & M & 13530869 \\
\hline $147 \mathrm{~V} 000067$ & 003307.22 & 483129.62 & 20.25 & 16.28 & 394 & 0.59 & 5.1 & - & - & $\mathrm{C}$ & 13541407 \\
\hline $147 \mathrm{~V} 000068$ & 003306.92 & 482820.66 & 20.06 & 18.01 & 167 & 0.53 & 5.9 & - & - & $\mathrm{M}$ & 13610395 \\
\hline $147 \mathrm{~V} 000069$ & 003307.05 & 483105.04 & 20.25 & 17.24 & 200 & 1.20 & 5.8 & - & - & $\mathrm{u}$ & 13621275 \\
\hline $147 \mathrm{~V} 000070$ & 003306.84 & 482808.27 & 19.93 & 16.92 & 260 & 0.50 & 6.4 & - & - & $\mathrm{C}$ & 13650329 \\
\hline 147V000071 & 003306.74 & 482801.40 & 19.73 & 16.94 & 317 & 0.75 & 6.0 & - & - & $\mathrm{C}$ & 13700292 \\
\hline $147 \mathrm{~V} 000072$ & 003306.83 & 483226.95 & 19.77 & 16.42 & 319 & 0.30 & 4.8 & - & - & $\mathrm{C}$ & 13771714 \\
\hline $147 \mathrm{~V} 000073$ & 003306.61 & 483135.60 & 20.04 & 16.84 & 223 & 0.95 & 6.4 & - & - & $\mathrm{C}$ & 13861439 \\
\hline $147 \mathrm{~V} 000074$ & 003306.44 & 483027.95 & 19.51 & 15.78 & 386 & 1.06 & 6.5 & - & - & M & 13921076 \\
\hline $147 \mathrm{~V} 000075$ & 003306.15 & 482814.38 & 20.33 & - & 194 & 0.85 & 6.0 & - & - & $\mathrm{C}$ & 14020361 \\
\hline $147 \mathrm{~V} 000076$ & 003305.73 & 482850.21 & 19.64 & 16.90 & 304 & 0.40 & 6.1 & - & - & $\mathrm{C}$ & 14260553 \\
\hline $147 \mathrm{~V} 000077$ & 003305.36 & 482843.42 & 19.93 & 18.05 & 159 & 0.88 & 4.6 & - & - & M & 14450516 \\
\hline $147 \mathrm{~V} 000078$ & 003305.32 & $4831 \quad 17.48$ & 19.75 & 17.03 & 208 & 0.63 & 5.4 & - & - & $\mathrm{S}$ & 14541341 \\
\hline $147 \mathrm{~V} 000079$ & 003304.98 & 482947.57 & 20.25 & 16.94 & 235 & 0.57 & 5.2 & - & - & M & 14680860 \\
\hline 147V000080 & 003304.72 & 482936.61 & 20.17 & 17.82 & 160 & 0.45 & 4.3 & - & - & M & 14810801 \\
\hline 147V000081 & 003304.81 & 483249.75 & 20.14 & 17.19 & 179 & 0.78 & 5.7 & - & - & M & 14851835 \\
\hline 147V000082 & 003304.50 & 482926.70 & 19.78 & 17.34 & 152 & 0.76 & 6.4 & - & - & M & 14920748 \\
\hline $147 \mathrm{~V} 000083$ & 003303.50 & 482753.84 & 20.44 & 17.11 & 234 & 0.99 & 5.8 & - & - & $\mathrm{C}$ & 15410250 \\
\hline 147V000084 & 003303.44 & 483032.32 & 20.07 & 17.01 & 258 & 0.58 & 6.0 & - & - & $\mathrm{C}$ & 15511099 \\
\hline $147 \mathrm{~V} 000085$ & 003303.47 & 483224.17 & 19.53 & 16.15 & 303 & 0.99 & 6.9 & - & - & $\mathrm{C}$ & 15551698 \\
\hline $147 \mathrm{~V} 000086$ & 003303.04 & 483031.54 & 19.74 & 16.80 & 248 & 0.43 & 5.1 & - & - & M & 15721095 \\
\hline $147 \mathrm{~V} 000087$ & 003302.87 & 483054.07 & 20.01 & 17.53 & 196 & 0.57 & 4.9 & - & - & M & 15821215 \\
\hline $147 \mathrm{~V} 000088$ & 003302.46 & 483127.43 & 19.88 & 17.09 & 234 & 0.36 & 4.2 & - & - & M & 16061394 \\
\hline 147V000089 & 003302.44 & 483123.39 & 20.17 & 18.12 & 157 & 1.13 & 4.0 & - & - & $\mathrm{S}$ & 16071372 \\
\hline $147 \mathrm{~V} 000090$ & 003302.24 & 483043.61 & 20.48 & 17.52 & 421 & 0.44 & 4.4 & - & - & M & 16151159 \\
\hline 147V000091 & 003302.11 & 483013.02 & 20.49 & 17.57 & 186 & 0.78 & 5.6 & - & - & M & 16210995 \\
\hline 147V000092 & 003301.88 & 482939.69 & 19.73 & 17.07 & 278 & 0.54 & 6.1 & - & - & $\mathrm{C}$ & 16310817 \\
\hline 147V000093 & 003301.12 & 482758.71 & 20.23 & 17.72 & 165 & 0.73 & 5.8 & - & - & $\mathrm{C}$ & 16670275 \\
\hline $147 \mathrm{~V} 000094$ & 003301.14 & 483105.04 & 20.06 & 17.55 & 168 & 0.75 & 5.4 & - & - & $\mathrm{S}$ & 16741274 \\
\hline $147 \mathrm{~V} 000095$ & 003301.01 & 482845.23 & 20.11 & 17.74 & 193 & 0.62 & 4.6 & - & - & S & 16750524 \\
\hline $147 \mathrm{~V} 000096$ & 003258.55 & 483049.40 & 20.53 & 17.34 & 200 & 1.05 & 5.7 & - & - & $\mathrm{u}$ & 18111189 \\
\hline 147V000097 & 003258.26 & 483110.67 & 20.07 & - & 161 & 0.65 & 5.5 & - & - & $\mathrm{S}$ & 18271303 \\
\hline $147 \mathrm{~V} 000098$ & 003258.02 & 483150.20 & 20.54 & 17.66 & 214 & 1.14 & 4.4 & - & - & $\mathrm{C}$ & 18421515 \\
\hline 147V000099 & 003257.77 & 482915.81 & 20.19 & 17.79 & 179 & 0.65 & 4.3 & - & - & M & 18480687 \\
\hline 147V000100 & 003257.48 & 482936.95 & 20.04 & 17.81 & 185 & 0.88 & 5.4 & - & - & M & 18640800 \\
\hline 147V000101 & 003257.19 & 482926.57 & 20.34 & 17.46 & 206 & 0.85 & 5.1 & - & - & $\mathrm{C}$ & 18790745 \\
\hline 147V000102 & 003257.06 & 483106.58 & 20.07 & 17.42 & 206 & 0.92 & 4.8 & - & - & $\mathrm{S}$ & 18911281 \\
\hline $147 \mathrm{~V} 000103$ & 003257.06 & 483249.75 & 20.64 & - & 180 & 1.26 & 4.5 & - & - & $\mathrm{u}$ & 18951834 \\
\hline 147V000104 & 003323.57 & 483034.46 & 19.51 & 17.13 & 270 & 0.89 & 6.4 & - & - & $\mathrm{u}$ & - \\
\hline 147V000105 & 003317.39 & 483115.19 & 19.94 & 17.36 & 238 & 1.15 & 4.6 & - & - & $\mathrm{u}$ & - \\
\hline 147V000106 & 003309.47 & 483150.70 & 19.69 & 17.15 & 211 & 0.64 & 5.3 & - & - & $\mathrm{u}$ & - \\
\hline 147V000107 & 003309.20 & 482727.16 & 20.16 & 16.77 & 271 & 1.08 & 2.9 & - & - & $\mathrm{u}$ & - \\
\hline 147V000108 & 003304.56 & 482921.92 & 19.72 & 17.22 & 387 & 0.46 & 4.2 & - & - & $\mathrm{u}$ & - \\
\hline 147V000109 & 003329.20 & 483045.91 & 20.51 & - & 270 & 1.09 & 3.6 & - & - & $\mathrm{u}$ & - \\
\hline 147V000110 & 003326.54 & 483248.50 & 19.93 & 17.12 & 219 & 0.63 & 7.7 & - & - & $\mathrm{S}$ & 03341833 \\
\hline 147V000111 & 003326.41 & 483140.98 & 20.61 & 16.71 & 321 & 0.86 & 7.3 & - & - & $\mathrm{C}$ & 03381473 \\
\hline 147V000112 & 003325.68 & $4833 \quad 15.14$ & 21.92 & 16.09 & 406 & 1.71 & 4.4 & - & - & $\mathrm{u}$ & - \\
\hline $147 \mathrm{~V} 000113$ & 003325.27 & 482954.83 & 19.76 & - & 140 & 0.38 & 3.5 & - & - & $\mathrm{u}$ & - \\
\hline $147 \mathrm{~V} 000114$ & 003324.44 & 483206.63 & 21.38 & 17.15 & 226 & 1.25 & 4.3 & - & - & $\mathrm{u}$ & - \\
\hline 147V000115 & 003324.26 & 482843.27 & 19.86 & 17.50 & 209 & 0.44 & 7.0 & - & - & $\mathrm{C}$ & 04450523 \\
\hline 147V000116 & 003323.93 & 483256.76 & 20.54 & - & 149 & 0.40 & 2.9 & - & - & $\mathrm{S}$ & 04731877 \\
\hline 147V000117 & 003323.75 & 483246.45 & 19.96 & 17.07 & 98 & 0.47 & 4.0 & - & - & M & 04821822 \\
\hline 147V000118 & 003323.38 & 483254.19 & 21.67 & - & 298 & 1.58 & 5.6 & - & - & $\mathrm{u}$ & - \\
\hline 147V000119 & 003322.53 & 482855.57 & 19.94 & 16.73 & 304 & 0.82 & 6.5 & - & - & $\mathrm{u}$ & - \\
\hline 147V000120 & 003321.94 & 483056.48 & 21.40 & - & 124 & 0.78 & 2.4 & - & - & $\mathrm{u}$ & - \\
\hline 147V000121 & 003321.89 & 483026.45 & 20.07 & 17.96 & 110 & 0.38 & 4.7 & - & - & $\mathrm{S}$ & 05751073 \\
\hline $147 \mathrm{~V} 000122$ & 003320.41 & 482941.12 & 19.93 & - & 143 & 0.34 & 5.1 & - & - & $\mathrm{u}$ & 06510831 \\
\hline $147 \mathrm{~V} 000123$ & 003319.94 & 482958.91 & 20.25 & 17.48 & 226 & 1.24 & 8.4 & - & - & $\mathrm{u}$ & - \\
\hline $147 \mathrm{~V} 000124$ & 003316.79 & 483141.36 & 20.52 & 15.86 & 438 & 1.20 & 5.7 & - & - & $\mathrm{u}$ & - \\
\hline $147 \mathrm{~V} 000125$ & 003316.82 & 483251.88 & 21.99 & - & 88 & 1.02 & 1.7 & - & - & $\mathrm{u}$ & 08491849 \\
\hline 147V000126 & 003316.69 & 483249.67 & 22.21 & - & 313 & 1.12 & 6.2 & - & - & $\mathrm{u}$ & 08551837 \\
\hline 147V000127 & 003316.49 & 483138.27 & 20.64 & 16.65 & 406 & 1.05 & 4.2 & - & - & $\mathrm{u}$ & - \\
\hline $147 \mathrm{~V} 000128$ & 003316.26 & 482804.49 & 20.08 & 17.20 & 223 & 0.36 & 5.3 & - & - & $\mathrm{u}$ & - \\
\hline $147 \mathrm{~V} 000129$ & 003316.31 & 483123.08 & 20.41 & 16.51 & 341 & 0.83 & 6.0 & - & - & $\mathrm{u}$ & - \\
\hline
\end{tabular}


Table A.1. continued.

\begin{tabular}{|c|c|c|c|c|c|c|c|c|c|c|c|}
\hline ID & $\begin{array}{c}\text { RAJ2000 } \\
\text { h:m:s }\end{array}$ & $\begin{array}{l}\text { DEJ2000 } \\
\text { d:m:s }\end{array}$ & $\begin{array}{c}i \\
{[\mathrm{mag}]}\end{array}$ & $\begin{array}{c}K_{\mathrm{s}} \\
{[\mathrm{mag}]}\end{array}$ & $\begin{array}{l}P_{0} \\
{[\mathrm{~d}]}\end{array}$ & $\begin{array}{c}\Delta i \\
{[\mathrm{mag}]}\end{array}$ & $s i g_{0}$ & $\begin{array}{l}P_{1} \\
{[\mathrm{~d}]}\end{array}$ & $s i g_{1}$ & type & ID \\
\hline $147 \mathrm{~V} 000130$ & $0033 \quad 15.33$ & 483050.77 & 20.48 & 17.99 & 143 & 1.05 & 4.6 & - & - & $\bar{S}$ & 09231201 \\
\hline 147V000131 & 003314.93 & 483323.72 & 20.24 & 17.22 & 200 & 0.71 & 6.3 & - & - & M & 09502019 \\
\hline $147 \mathrm{~V} 000132$ & 003314.24 & 483112.86 & 19.98 & 17.84 & 157 & 0.57 & 4.8 & - & - & $\mathrm{S}$ & 09811319 \\
\hline $147 \mathrm{~V} 000133$ & 003312.79 & 483102.92 & 20.70 & 16.51 & 379 & 1.01 & 8.6 & - & - & $\mathrm{u}$ & - \\
\hline $147 \mathrm{~V} 000134$ & 003312.64 & 482959.52 & 19.71 & 16.83 & 246 & 0.34 & 5.3 & - & - & $\mathrm{C}$ & 10630926 \\
\hline 147V000135 & 003312.58 & 483101.90 & 20.81 & 16.53 & 346 & 1.47 & 4.4 & - & - & $\mathrm{u}$ & - \\
\hline $147 \mathrm{~V} 000136$ & 003312.36 & 483029.70 & 20.33 & 17.10 & 257 & 1.88 & 6.7 & - & - & $\mathrm{u}$ & - \\
\hline 147V000137 & 003311.09 & 482848.81 & 20.04 & 16.60 & 178 & 0.88 & 5.7 & - & - & $\mathrm{u}$ & - \\
\hline $147 \mathrm{~V} 000138$ & 003309.02 & 482950.59 & 21.02 & - & 133 & 0.88 & 6.6 & - & - & $\mathrm{u}$ & - \\
\hline $147 \mathrm{~V} 000139$ & 003308.32 & 482926.70 & 20.24 & 17.09 & 109 & 0.58 & 5.2 & - & - & $\mathrm{u}$ & - \\
\hline $147 \mathrm{~V} 000140$ & 003308.07 & 482821.69 & 19.84 & 17.16 & 202 & 0.38 & 5.2 & - & - & M & 13000401 \\
\hline 147V000141 & 003307.87 & 483132.07 & 20.11 & 17.15 & 186 & 0.41 & 3.7 & - & - & $\mathrm{u}$ & - \\
\hline 147V000142 & 003307.19 & 483237.74 & 20.11 & 17.48 & 145 & 0.53 & 6.0 & - & - & M & 13581771 \\
\hline $147 \mathrm{~V} 000143$ & 003306.40 & 482955.81 & 20.65 & 17.37 & 525 & 0.57 & 4.2 & - & - & $\mathrm{S}$ & 13930904 \\
\hline 147V000144 & 003302.05 & 482947.60 & 20.16 & 16.82 & 218 & 0.62 & 4.0 & - & - & $\mathrm{u}$ & - \\
\hline $147 \mathrm{~V} 000145$ & 003301.85 & 483006.08 & 20.23 & 17.61 & 153 & 0.64 & 5.7 & - & - & $\mathrm{u}$ & - \\
\hline $147 \mathrm{~V} 000146$ & 003258.61 & 482946.87 & 19.64 & 17.36 & 184 & 0.42 & 5.5 & - & - & M & 18050854 \\
\hline 00147 & 003257.69 & 482741.80 & 20.10 & 16.76 & 254 & 0.58 & 4.5 & - & - & $\mathrm{u}$ & 18480183 \\
\hline $147 \mathrm{~V} 000148$ & 003304.22 & 482912.83 & 19.42 & 16.15 & 348 & 0.35 & 4.5 & - & - & $\mathrm{C}$ & 15070673 \\
\hline 147V000149 & 003314.36 & 483121.40 & 19.92 & 16.34 & 345 & 0.56 & 4.3 & 690 & 3.0 & $\mathrm{~S}$ & 09751365 \\
\hline $147 \mathrm{~V} 000150$ & 003310.37 & 483006.46 & 20.38 & 16.33 & 406 & 0.78 & 6.2 & 813 & 0.7 & $\mathrm{u}$ & - \\
\hline $147 \mathrm{~V} 000151$ & 003320.48 & 483141.33 & 20.44 & 17.04 & 234 & 2.22 & 3.5 & 373 & 1.5 & M & 06521473 \\
\hline $147 \mathrm{~V} 000152$ & 003320.35 & 483218.83 & 19.52 & 16.70 & 222 & 0.46 & 4.5 & 804 & 0.8 & M & 06611673 \\
\hline $147 \mathrm{~V} 000153$ & 003314.07 & 483316.54 & 20.25 & 17.16 & 226 & 0.72 & 3.2 & 371 & 1.5 & $\mathrm{C}$ & 09951980 \\
\hline $147 \mathrm{~V} 000154$ & 003307.38 & 483038.03 & 20.40 & 17.26 & 178 & 0.93 & 4.2 & 238 & 1.4 & $\mathrm{C}$ & 13431131 \\
\hline 147V000155 & 003322.65 & 483223.44 & 19.95 & 16.74 & 267 & 0.61 & 5.0 & 293 & 2.5 & $\mathrm{C}$ & 05391698 \\
\hline 147V000156 & 003316.18 & 482819.31 & 19.75 & 16.43 & 280 & 0.50 & 4.9 & 363 & 0.7 & $\mathrm{C}$ & 08710392 \\
\hline $147 \mathrm{~V} 000157$ & 003312.89 & 482950.98 & 19.75 & 16.58 & 316 & 0.33 & 5.3 & 790 & 1.0 & $\mathrm{C}$ & 10500881 \\
\hline $147 \mathrm{~V} 000158$ & 003305.44 & 483018.10 & 20.49 & 16.71 & 226 & 1.31 & 4.9 & 293 & 0.9 & $\mathrm{C}$ & 14451023 \\
\hline $147 \mathrm{~V} 000159$ & 003302.32 & 482932.13 & 19.76 & 16.77 & 305 & 0.52 & 5.0 & 1023 & 1.2 & $\mathrm{C}$ & 16080776 \\
\hline $147 \mathrm{~V} 000160$ & 003300.92 & 482941.43 & 19.66 & 16.17 & 378 & 1.68 & 6.1 & 564 & 3.3 & M & 16820825 \\
\hline 147V000161 & 003300.66 & 482740.70 & 21.40 & - & 371 & 2.74 & 3.2 & 6 & .0 & $\mathrm{u}$ & 16900179 \\
\hline 147V000162 & 003259.44 & 482925.74 & 20.41 & 16.91 & 266 & 1.09 & 4.4 & 548 & 0.8 & $\mathrm{C}$ & 17600741 \\
\hline 147V000163 & 003256.70 & 482939.82 & 20.60 & 17.11 & 377 & 0.91 & 4.9 & 832 & 1.0 & $\mathrm{u}$ & 19060816 \\
\hline 147V000164 & 003304.76 & 482929.04 & 19.48 & 16.98 & 232 & 0.64 & 4.6 & 302 & 1.4 & $\mathrm{u}$ & - \\
\hline 147V000165 & 003322.99 & 483158.23 & 21.21 & 16.80 & 348 & 1.09 & 9.1 & 784 & 2.2 & $\mathrm{u}$ & - \\
\hline 147V000166 & 003316.37 & 482804.20 & 20.46 & 16.81 & 292 & 0.79 & 8.1 & 822 & 3.5 & $\mathrm{u}$ & - \\
\hline 147V000167 & 003307.47 & 483026.76 & 20.09 & 17.25 & 250 & 1.29 & 7.2 & 388 & 5.6 & $\mathrm{u}$ & - \\
\hline 147V000168 & 003304.17 & 482859.64 & 20.22 & 16.80 & 304 & 1.49 & 7.2 & 822 & 4.7 & $\mathrm{u}$ & - \\
\hline 147V000169 & 003318.39 & 483101.83 & 20.06 & 17.11 & - & 0.55 & - & - & - & M & 07611261 \\
\hline $147 \mathrm{~V} 000170$ & 003317.44 & 483141.06 & 21.09 & 16.22 & - & 1.63 & - & - & - & $\mathrm{S}$ & 08131471 \\
\hline 147V000171 & 003305.58 & 482837.77 & 21.54 & 17.33 & - & 1.39 & - & - & - & C & 14330486 \\
\hline 147V000172 & 003327.70 & 482935.30 & 19.78 & 17.36 & - & 0.73 & - & - & - & M & 02650802 \\
\hline $147 \mathrm{~V} 000173$ & 003326.44 & 483150.12 & 20.02 & - & - & 0.44 & - & - & - & M & 03371522 \\
\hline $147 \mathrm{~V} 000174$ & 23.51 & 483041.14 & 19.71 & 17.25 & - & 0.26 & - & - & - & M & 04891152 \\
\hline $147 \mathrm{~V} 000175$ & 0033 & 482834.61 & 19.96 & - & - & 0.56 & - & - & - & M & 06210475 \\
\hline $147 \mathrm{~V} 000176$ & 003320.07 & 483317.28 & 20.01 & 17.36 & - & 0.61 & - & - & - & $\mathrm{S}$ & 06781986 \\
\hline $147 \mathrm{~V} 000177$ & 003318.96 & 483025.85 & 19.70 & 16.75 & - & 0.50 & - & - & - & M & 07291069 \\
\hline $147 \mathrm{~V} 000178$ & 003317.45 & 482755.86 & 19.90 & 16.75 & - & 0.46 & - & - & - & M & 08030267 \\
\hline $147 \mathrm{~V} 000179$ & 003317.18 & 482847.15 & 19.75 & 17.27 & - & 0.34 & - & - & - & $\mathrm{u}$ & - \\
\hline $147 \mathrm{~V} 000180$ & 003316.90 & 483057.62 & 19.98 & 17.63 & - & 0.54 & - & - & - & S & 08401238 \\
\hline 147V000181 & 003313.63 & 482924.23 & 20.09 & 17.06 & - & 0.39 & - & - & - & $\mathrm{u}$ & - \\
\hline $147 \mathrm{~V} 000182$ & 003313.46 & 483055.17 & 20.01 & 16.83 & - & 0.44 & - & - & - & $\mathrm{C}$ & 10221224 \\
\hline $147 \mathrm{~V} 000183$ & 003312.63 & 483043.45 & 19.83 & 17.01 & - & 0.48 & - & - & - & $\mathrm{u}$ & - \\
\hline 147V000184 & 003310.85 & 482954.19 & 19.69 & 17.23 & - & 0.53 & - & - & - & $\mathrm{C}$ & 11570897 \\
\hline 147V000185 & 003310.70 & 483130.87 & 20.54 & 16.99 & - & 1.67 & - & - & - & $\mathrm{S}$ & - \\
\hline $147 \mathrm{~V} 000186$ & 003309.98 & 483218.45 & 20.10 & 17.84 & - & 0.49 & - & - & - & $\mathrm{C}$ & 12091669 \\
\hline $147 \mathrm{~V} 000187$ & 003309.34 & 483207.84 & 20.28 & 16.67 & - & 0.75 & - & - & - & $\mathrm{C}$ & 12431612 \\
\hline $147 \mathrm{~V} 000188$ & 003308.27 & 483243.73 & 20.24 & 17.63 & - & 0.44 & - & - & - & $\mathrm{u}$ & - \\
\hline 147V000189 & 003306.62 & 483049.13 & 19.84 & 18.09 & - & 0.60 & - & - & - & $\mathrm{S}$ & 13841190 \\
\hline $147 \mathrm{~V} 000190$ & 003305.96 & 483319.34 & 19.66 & 18.04 & - & 0.36 & - & - & - & S & 14261994 \\
\hline 147V000191 & 003305.62 & 483219.62 & 20.01 & 17.01 & - & 0.60 & - & - & - & $\mathrm{u}$ & - \\
\hline 147V000192 & 003305.50 & 483052.88 & 19.28 & 16.20 & - & 0.37 & - & - & - & $\mathrm{C}$ & 14431210 \\
\hline $147 \mathrm{~V} 000193$ & 003302.33 & 483252.16 & 20.12 & 16.78 & - & 0.81 & - & - & - & $\mathrm{C}$ & 16161848 \\
\hline 147V000194 & 003301.70 & 483031.87 & 19.78 & 16.78 & - & 0.30 & - & - & - & M & 16441096 \\
\hline
\end{tabular}


Table A.1. continued.

\begin{tabular}{|c|c|c|c|c|c|c|c|c|c|c|c|}
\hline ID & $\begin{array}{c}\text { RAJ2000 } \\
\text { h:m:s }\end{array}$ & $\begin{array}{l}\text { DEJ2000 } \\
\text { d:m:s }\end{array}$ & $\begin{array}{c}i \\
{[\mathrm{mag}]}\end{array}$ & $\begin{array}{c}K_{\mathrm{s}} \\
{[\mathrm{mag}]}\end{array}$ & $\begin{array}{l}P_{0} \\
{[\mathrm{~d}]}\end{array}$ & $\begin{array}{c}\Delta i \\
{[\mathrm{mag}]}\end{array}$ & $\operatorname{sig}_{0}$ & $\begin{array}{l}P_{1} \\
{[\mathrm{~d}]}\end{array}$ & $\operatorname{sig}_{1}$ & type & ID ${ }_{\text {Paper I }}$ \\
\hline $147 \mathrm{~V} 000195$ & 003258.91 & 483108.84 & 20.30 & 17.64 & - & 0.67 & $\overline{-}$ & - & $\overline{-}$ & $\mathrm{S}$ & 17931293 \\
\hline 147V000196 & 003258.00 & 482828.95 & 20.09 & 17.86 & - & 0.51 & - & - & - & $\mathrm{M}$ & 18330436 \\
\hline 147V000197 & 003308.90 & 482926.45 & 21.20 & - & - & 1.70 & - & - & - & $\mathrm{u}$ & - \\
\hline 147V000198 & 003304.84 & 482904.69 & 21.17 & - & - & 1.64 & - & - & - & $\mathrm{u}$ & - \\
\hline 147V000199 & 003327.10 & 482949.27 & 21.28 & - & - & 0.75 & - & - & - & $\mathrm{u}$ & - \\
\hline $147 \mathrm{~V} 000200$ & 323.90 & 483032.65 & 21.31 & - & - & 0.71 & - & - & - & $\mathrm{u}$ & 04691107 \\
\hline 000201 & 3.28 & 9.71 & 20.90 & - & - & 0.59 & - & - & - & $\mathrm{u}$ & 05011145 \\
\hline $147 \mathrm{~V} 000202$ & 03323.07 & 483152.27 & 20.24 & 16.87 & - & 0.53 & - & - & 一 & $\mathrm{u}$ & - \\
\hline 147V000203 & 003322.59 & 482937.69 & 20.75 & - & - & 0.62 & - & - & - & S & 05350813 \\
\hline 147V000204 & 003321.17 & 482837.66 & 21.64 & - & - & 0.99 & - & - & - & $\mathrm{u}$ & - \\
\hline 147V000205 & 003318.20 & 483050.04 & 21.13 & 17.85 & - & 1.16 & - & - & - & $\mathrm{u}$ & - \\
\hline 147V000206 & 003316.65 & 483051.67 & 20.01 & 17.86 & - & 0.30 & - & - & - & $\mathrm{C}$ & 08531206 \\
\hline $147 \mathrm{~V} 000207$ & 003312.08 & 483111.36 & 19.74 & 16.41 & - & 0.43 & - & - & - & $\mathrm{u}$ & - \\
\hline 147V000208 & 003311.67 & 482926.87 & 20.10 & 17.69 & - & 0.42 & - & - & - & M & 11130751 \\
\hline 147V000209 & 003308.64 & $4832 \quad 15.42$ & 21.41 & 18.31 & - & 1.16 & - & - & - & $\mathrm{u}$ & - \\
\hline 147V000210 & 003303.98 & 483108.43 & 21.46 & - & - & 1.12 & - & - & - & $\mathrm{u}$ & - \\
\hline 147V000211 & 003302.06 & 482812.73 & 20.26 & 19.19 & - & 0.49 & - & - & - & $\mathrm{u}$ & - \\
\hline 147V000212 & 003301.44 & 483003.94 & 20.12 & 17.01 & - & 0.45 & - & - & - & $\mathrm{u}$ & - \\
\hline $147 \mathrm{~V} 000213$ & 003300.88 & 482920.82 & 19.72 & 16.98 & - & 0.26 & - & - & - & M & 16840715 \\
\hline
\end{tabular}

Notes. This table lists in the first five columns the internal IDs, the coordinates (J2000), mean $i$ magnitudes (obtained from the time series) as well as $K_{\mathrm{s}}$-magnitudes. Periods, $\sigma$-amplitudes, and the SIGSPEC-significance are found in the next three columns. Long-period variables exhibiting two significant periods have entries in the ninth and tenth columns listing the second period and its SIGSPEC-significance. The last two columns give the types of different chemistry and the IDs carried out by the authors of Paper I. 
Table A.2. Same as Table A.1 but for NGC 185.

\begin{tabular}{|c|c|c|c|c|c|c|c|c|c|c|c|}
\hline ID & $\begin{array}{c}\text { RAJ2000 } \\
\text { h:m:s }\end{array}$ & $\begin{array}{c}\text { DEJ2000 } \\
\text { d:m:s }\end{array}$ & $\begin{array}{c}i \\
{[\mathrm{mag}]}\end{array}$ & $\begin{array}{c}K_{\mathrm{s}} \\
{[\mathrm{mag}]}\end{array}$ & $\begin{array}{l}P_{0} \\
{[\mathrm{~d}]}\end{array}$ & $\begin{array}{c}\Delta i \\
{[\mathrm{mag}]}\end{array}$ & $s i g_{0}$ & $\begin{array}{l}P_{1} \\
{[\mathrm{~d}]}\end{array}$ & $\operatorname{sig}_{1}$ & type & ID ${ }_{\text {Paper I }}$ \\
\hline $185 \mathrm{~V} 000001$ & 003914.67 & 482040.97 & 19.68 & - & 139 & 0.43 & 4.0 & - & - & $\mathrm{S}$ & 01961242 \\
\hline $185 \mathrm{~V} 000002$ & 003914.42 & 481837.24 & 19.84 & - & 114 & 0.43 & 4.4 & - & - & $\mathrm{S}$ & 02020584 \\
\hline $185 \mathrm{~V} 000003$ & 003914.12 & 482131.49 & 20.03 & - & 153 & 0.66 & 2.8 & - & - & M & 02281511 \\
\hline $185 \mathrm{~V} 000004$ & 003913.29 & 482028.43 & 19.58 & - & 147 & 0.55 & 5.7 & - & - & M & 02681175 \\
\hline $185 \mathrm{~V} 000005$ & 003852.03 & 482010.21 & 19.28 & 16.31 & 371 & 0.46 & 6.0 & - & - & $\mathrm{C}$ & 13961070 \\
\hline $185 \mathrm{~V} 000006$ & 003913.12 & 481925.54 & 19.57 & 17.48 & 163 & 0.46 & 5.6 & - & - & $\mathrm{S}$ & 02740840 \\
\hline 185V000007 & 003912.54 & 481754.78 & 20.41 & - & 311 & 0.84 & 6.1 & - & - & $\mathrm{u}$ & 02990357 \\
\hline $185 \mathrm{~V} 000008$ & 003912.44 & 482106.86 & 20.41 & - & 123 & 0.86 & 3.8 & - & - & M & 03161379 \\
\hline $185 \mathrm{~V} 000009$ & 003911.84 & 481943.23 & 20.18 & 16.89 & 277 & 0.72 & 5.9 & - & - & $\mathrm{C}$ & 03430934 \\
\hline $185 \mathrm{~V} 000010$ & 003911.74 & 482111.84 & 19.70 & 18.04 & 169 & 0.91 & 5.9 & - & - & M & 03531405 \\
\hline $185 \mathrm{~V} 000011$ & 003911.78 & 482146.36 & 19.31 & 17.54 & 226 & 0.46 & 4.8 & - & - & M & 03531589 \\
\hline $185 \mathrm{~V} 000012$ & 003911.23 & 481953.03 & 20.06 & - & 109 & 0.48 & 4.5 & - & - & $\mathrm{S}$ & 03760986 \\
\hline $185 \mathrm{~V} 000013$ & 003911.12 & 482020.59 & 19.99 & 17.85 & 124 & 0.58 & 5.0 & - & - & M & 03831132 \\
\hline $185 \mathrm{~V} 000014$ & 003910.87 & 482017.89 & 19.58 & 17.09 & 588 & 0.31 & 3.7 & - & - & M & 03961118 \\
\hline $185 \mathrm{~V} 000015$ & 003910.41 & 482052.43 & 20.78 & - & 168 & 1.07 & 4.9 & - & - & M & 04231301 \\
\hline $185 \mathrm{~V} 000016$ & 003909.69 & 481944.06 & 20.35 & - & 120 & 0.87 & 4.9 & - & - & $\mathrm{S}$ & 04570937 \\
\hline 185V000017 & 003909.65 & 481942.87 & 19.67 & - & 203 & 0.87 & 6.1 & - & - & $\mathrm{S}$ & 04590931 \\
\hline $185 \mathrm{~V} 000018$ & 003909.79 & 482101.08 & 19.66 & - & 142 & 0.29 & 3.9 & - & - & M & 04561347 \\
\hline $185 \mathrm{~V} 000019$ & 003909.34 & 482026.12 & 19.68 & 17.93 & 144 & 0.56 & 4.4 & - & - & M & 04781161 \\
\hline $185 \mathrm{~V} 000020$ & 003909.09 & 481842.08 & 19.27 & 17.25 & 200 & 0.43 & 5.3 & - & - & M & 04860607 \\
\hline $185 \mathrm{~V} 000021$ & 003909.18 & 481959.60 & 19.47 & 17.24 & 240 & 0.41 & 6.3 & - & - & $\mathrm{C}$ & 04851020 \\
\hline $185 \mathrm{~V} 000022$ & 003909.03 & 481951.86 & 19.95 & 16.54 & 301 & 0.76 & 6.0 & - & - & $\mathrm{C}$ & 04930978 \\
\hline $185 \mathrm{~V} 000023$ & 003909.17 & 482256.02 & 19.51 & 17.21 & 204 & 0.68 & 5.8 & - & - & $\mathrm{S}$ & 04951959 \\
\hline $185 \mathrm{~V} 000024$ & 003908.91 & 482300.90 & 19.43 & 17.21 & 185 & 0.32 & 3.4 & - & - & M & 05091985 \\
\hline $185 \mathrm{~V} 000025$ & 003908.53 & 481850.95 & 19.55 & 16.96 & 249 & 0.57 & 5.6 & - & - & $\mathrm{C}$ & 05160654 \\
\hline $185 \mathrm{~V} 000026$ & 003908.46 & 482122.09 & 20.30 & 16.40 & 421 & 0.48 & 4.4 & - & - & $\mathrm{C}$ & 05281459 \\
\hline $185 \mathrm{~V} 000027$ & 003908.51 & 482217.14 & 19.89 & 17.50 & 391 & 0.41 & 4.5 & - & - & M & 05281752 \\
\hline 185V000028 & 003908.35 & 481957.81 & 19.73 & 17.50 & 169 & 0.95 & 5.7 & - & - & M & 05291010 \\
\hline $185 \mathrm{~V} 000029$ & 003908.33 & 482108.51 & 20.55 & 16.69 & 338 & 0.90 & 4.8 & - & - & $\mathrm{C}$ & 05341386 \\
\hline $185 \mathrm{~V} 000030$ & 003908.18 & 481939.03 & 19.32 & 17.45 & 168 & 0.55 & 6.4 & - & - & $\mathrm{S}$ & 05370910 \\
\hline $185 \mathrm{~V} 000031$ & 003908.23 & 482125.98 & 19.70 & 17.72 & 186 & 0.73 & 4.0 & - & - & M & 05401479 \\
\hline $185 \mathrm{~V} 000032$ & 003908.15 & 482121.75 & 19.35 & 17.27 & 176 & 0.33 & 2.7 & - & - & M & 05441457 \\
\hline $185 \mathrm{~V} 000033$ & 003907.84 & $48 \quad 1721.27$ & 19.55 & - & 148 & 0.59 & 5.1 & - & - & M & 05480176 \\
\hline $185 \mathrm{~V} 000034$ & 003907.79 & 482034.30 & 19.25 & 16.63 & 137 & 0.27 & 4.0 & - & - & M & 05611204 \\
\hline $185 \mathrm{~V} 000035$ & 003907.46 & 482020.22 & 19.97 & 17.78 & 154 & 0.53 & 4.7 & - & - & $\mathrm{S}$ & 05771129 \\
\hline $185 \mathrm{~V} 000036$ & 003907.47 & 482228.94 & 20.04 & 16.70 & 305 & 0.74 & 5.1 & - & - & $\mathrm{u}$ & 05841814 \\
\hline $185 \mathrm{~V} 000037$ & 003907.19 & 481904.86 & 19.69 & 17.34 & 215 & 0.61 & 4.3 & - & - & M & 05880728 \\
\hline $185 \mathrm{~V} 000038$ & 003907.08 & 482216.59 & 19.50 & 17.32 & 195 & 0.40 & 5.7 & - & - & M & 06041748 \\
\hline $185 \mathrm{~V} 000039$ & 003906.81 & 481830.65 & 20.25 & 17.52 & 217 & 1.16 & 6.6 & - & - & $\mathrm{u}$ & - \\
\hline $185 \mathrm{~V} 000040$ & 003906.67 & 481715.92 & 19.65 & - & 313 & 0.88 & 3.5 & - & - & $\mathrm{u}$ & 06090147 \\
\hline 185V000041 & 003906.77 & 482146.92 & 19.80 & 17.29 & 237 & 1.14 & 6.7 & - & - & M & 06181590 \\
\hline $185 \mathrm{~V} 000042$ & 003906.67 & 482251.48 & 19.30 & 17.19 & 172 & 0.65 & 5.3 & - & - & M & 06271934 \\
\hline $185 \mathrm{~V} 000043$ & 003906.69 & 482305.96 & 19.73 & 16.89 & 288 & 0.78 & 4.7 & - & - & $\mathrm{C}$ & 06272011 \\
\hline $185 \mathrm{~V} 000044$ & 003906.36 & 481823.61 & 19.50 & 17.33 & 199 & 0.39 & 5.9 & - & - & M & 06300508 \\
\hline $185 \mathrm{~V} 000045$ & 003906.40 & 481914.30 & 20.37 & 16.91 & 326 & 1.42 & 6.1 & - & - & $\mathrm{C}$ & 06300777 \\
\hline 185V000046 & 003906.36 & 481919.69 & 20.04 & - & 128 & 0.77 & 6.1 & - & - & $\mathrm{S}$ & 06320806 \\
\hline $185 \mathrm{~V} 000047$ & 003905.93 & 481754.05 & 20.10 & 17.28 & 189 & 0.51 & 5.8 & - & - & $\mathrm{C}$ & 06510350 \\
\hline $185 \mathrm{~V} 000048$ & 003906.05 & 482045.86 & 19.89 & 17.38 & 122 & 0.59 & 3.7 & - & - & M & 06541265 \\
\hline $185 \mathrm{~V} 000049$ & 003906.13 & 482256.61 & 19.32 & 16.01 & 399 & 1.06 & 5.0 & - & - & M & 06561961 \\
\hline $185 \mathrm{~V} 000050$ & 003905.92 & 482108.54 & 19.75 & 17.39 & 183 & 0.79 & 6.3 & - & - & M & 06621386 \\
\hline 185V000051 & 003905.68 & 482001.49 & 19.79 & - & 161 & 0.67 & 5.3 & - & - & M & 06711028 \\
\hline $185 \mathrm{~V} 000052$ & 003905.62 & 481932.81 & 20.01 & 17.21 & 247 & 0.70 & 5.9 & - & - & $\mathrm{C}$ & 06720876 \\
\hline $185 \mathrm{~V} 000053$ & 003905.50 & 482021.72 & 19.71 & 17.92 & 132 & 0.60 & 6.5 & - & - & M & 06811136 \\
\hline $185 \mathrm{~V} 000054$ & 003905.34 & 481935.25 & 19.12 & 16.92 & 95 & 0.19 & 3.9 & - & - & M & 06870889 \\
\hline $185 \mathrm{~V} 000055$ & 003905.35 & 482107.57 & 19.86 & 16.70 & 262 & 0.43 & 5.2 & - & - & M & 06921380 \\
\hline $185 \mathrm{~V} 000056$ & 003905.26 & 482119.33 & 20.30 & - & 133 & 1.15 & 5.3 & - & - & M & 06971443 \\
\hline $185 \mathrm{~V} 000057$ & 003905.12 & 482004.63 & 20.60 & 18.13 & 141 & 0.98 & 5.0 & - & - & M & 07011045 \\
\hline $185 \mathrm{~V} 000058$ & 003904.74 & 481816.68 & 19.60 & - & 107 & 0.33 & 3.5 & - & - & $\mathrm{S}$ & 07150470 \\
\hline $185 \mathrm{~V} 000059$ & 003904.86 & 482215.07 & 19.46 & 17.23 & 467 & 0.29 & 3.9 & - & - & M & 07211739 \\
\hline
\end{tabular}


Table A.2. continued.

\begin{tabular}{|c|c|c|c|c|c|c|c|c|c|c|c|}
\hline ID & $\begin{array}{c}\text { RAJ2000 } \\
\text { h:m:s }\end{array}$ & $\begin{array}{c}\text { DEJ2000 } \\
\text { d:m:s }\end{array}$ & $\begin{array}{c}i \\
{[\mathrm{mag}]}\end{array}$ & $\left.\begin{array}{c}K_{\mathrm{s}} \\
{[\mathrm{mag}]}\end{array}\right]$ & $\begin{array}{l}P_{0} \\
{[\mathrm{~d}]}\end{array}$ & $\begin{array}{c}\Delta i \\
{[\mathrm{mag}]}\end{array}$ & $s i g_{0}$ & $\begin{array}{l}P_{1} \\
{[\mathrm{~d}]}\end{array}$ & $s i g_{1}$ & type & ID ${ }_{\text {Paper I }}$ \\
\hline $185 \mathrm{~V} 000060$ & 003904.73 & 482050.01 & 19.83 & 16.78 & 229 & 1.15 & 6.6 & - & - & $\mathrm{S}$ & 07241286 \\
\hline 185V000061 & 003904.53 & 481912.11 & 19.47 & 16.83 & 252 & 0.49 & 6.0 & - & - & $\mathrm{C}$ & 07290765 \\
\hline $185 \mathrm{~V} 000062$ & 003904.43 & 482138.13 & 20.13 & 16.65 & 219 & 0.90 & 6.2 & - & - & $\mathrm{u}$ & 07421543 \\
\hline $185 \mathrm{~V} 000063$ & 003904.11 & 481800.35 & 20.33 & - & 107 & 0.78 & 3.9 & - & - & $\mathrm{S}$ & 07480383 \\
\hline $185 \mathrm{~V} 000064$ & 003904.18 & 482008.44 & 19.51 & 17.52 & 192 & 0.94 & 6.7 & - & - & M & 07501065 \\
\hline $185 \mathrm{~V} 000065$ & 003904.21 & 482219.71 & 20.14 & 17.73 & 146 & 0.72 & 4.5 & - & - & M & 07561764 \\
\hline $185 \mathrm{~V} 000066$ & 003903.99 & 482003.78 & 20.18 & 16.49 & 325 & 0.80 & 6.3 & - & - & $\mathrm{u}$ & 07601040 \\
\hline $185 \mathrm{~V} 000067$ & 003903.53 & 481906.36 & 19.72 & - & 92 & 0.59 & 4.2 & - & - & $\mathrm{S}$ & 07820734 \\
\hline $185 \mathrm{~V} 000068$ & 003841.55 & 481930.40 & 19.98 & 17.17 & 237 & 0.70 & 5.5 & - & - & $\mathrm{C}$ & 19500854 \\
\hline 185V000069 & 003842.10 & 481940.52 & 19.78 & 17.71 & 340 & 0.31 & 4.4 & - & - & M & 19210908 \\
\hline $185 \mathrm{~V} 000070$ & 003903.20 & 481806.89 & 19.87 & - & 157 & 0.51 & 5.5 & - & - & $\mathrm{u}$ & 07960417 \\
\hline $185 \mathrm{~V} 000071$ & 003903.33 & 482140.27 & 19.58 & 17.52 & 167 & 0.37 & 4.4 & - & - & M & 08011554 \\
\hline $185 \mathrm{~V} 000072$ & 003903.15 & 482033.42 & 19.39 & - & 140 & 0.50 & 5.0 & - & - & M & 08071198 \\
\hline $185 \mathrm{~V} 000073$ & 003903.21 & 482122.16 & 19.85 & 17.31 & 95 & 0.32 & 1.8 & - & - & M & 08061457 \\
\hline $185 \mathrm{~V} 000074$ & 003903.08 & 481947.34 & 19.69 & 16.41 & 286 & 0.49 & 4.2 & - & - & $\mathrm{S}$ & 08080952 \\
\hline $185 \mathrm{~V} 000075$ & 003902.69 & 482144.92 & 20.08 & - & 139 & 0.58 & 4.9 & - & - & M & 08351578 \\
\hline 185V000076 & 003902.52 & 481900.32 & 21.15 & 16.31 & 358 & 1.23 & 4.5 & - & - & $\mathrm{C}$ & 08350701 \\
\hline 185V000077 & 003902.58 & 482031.21 & 19.39 & - & 151 & 0.59 & 5.9 & - & - & $\mathrm{S}$ & 08371186 \\
\hline 185V000078 & 003902.28 & 481918.53 & 20.45 & 16.07 & 420 & 0.65 & 5.4 & - & - & $\mathrm{S}$ & 08490798 \\
\hline 185V000079 & 003902.29 & 482048.95 & 19.80 & - & 159 & 0.47 & 5.5 & - & - & M & 08531280 \\
\hline $185 \mathrm{~V} 000080$ & 003901.98 & 481738.70 & 19.70 & 17.11 & 225 & 0.43 & 5.4 & - & - & $\mathrm{C}$ & 08600266 \\
\hline 185V000081 & 003902.17 & 482100.07 & 19.61 & 17.47 & 210 & 0.73 & 6.2 & - & - & M & 08601339 \\
\hline 185V000082 & 003902.06 & 482056.13 & 19.25 & 16.90 & 235 & 0.58 & 6.3 & - & - & M & 08661318 \\
\hline $185 \mathrm{~V} 000083$ & 003901.77 & 481848.91 & 19.70 & 16.79 & 280 & 0.52 & 6.3 & - & - & $\mathrm{C}$ & 08740640 \\
\hline 185V000084 & 003901.92 & 482155.53 & 19.28 & 17.53 & 127 & 0.42 & 4.4 & - & - & $\mathrm{S}$ & 08761635 \\
\hline $185 \mathrm{~V} 000085$ & 003901.76 & 481934.17 & 18.93 & 16.42 & 239 & 0.22 & 4.1 & - & - & M & 08770881 \\
\hline 185V000086 & 003901.75 & 481932.45 & 19.48 & 16.82 & 222 & 0.56 & 6.2 & - & - & M & 08780872 \\
\hline 185V000087 & 003901.70 & 482038.90 & 19.75 & 16.84 & 131 & 0.41 & 3.9 & - & - & M & 08841226 \\
\hline 185V000088 & 003901.59 & 481907.12 & 19.65 & 16.32 & 339 & 0.56 & 5.2 & - & - & $\mathrm{S}$ & 08850737 \\
\hline 185V000089 & 003901.66 & 482308.84 & 19.38 & 17.17 & 183 & 0.32 & 3.1 & - & - & $\mathrm{S}$ & 08942025 \\
\hline $185 \mathrm{~V} 000090$ & 003901.01 & 481731.20 & 19.19 & 16.93 & 154 & 0.15 & 2.8 & - & - & M & 09110226 \\
\hline 185V000091 & 003900.93 & 481959.41 & 19.09 & 16.37 & 302 & 0.22 & 5.4 & - & - & $\mathrm{S}$ & 09231016 \\
\hline 185V000092 & 003900.98 & 482201.18 & 20.23 & 17.45 & 192 & 0.92 & 5.3 & - & - & M & 09271664 \\
\hline $185 \mathrm{~V} 000093$ & 003900.93 & 482148.81 & 19.63 & 16.37 & 280 & 0.69 & 6.6 & - & - & $\mathrm{C}$ & 09281598 \\
\hline $185 \mathrm{~V} 000094$ & 003900.91 & 482133.04 & 20.14 & 16.78 & 236 & 0.85 & 5.2 & - & - & $\mathrm{C}$ & 09281514 \\
\hline 185V000095 & 003900.68 & 481901.60 & 20.16 & 16.56 & 315 & 0.70 & 4.9 & - & - & $\mathrm{u}$ & 09330707 \\
\hline 185V000096 & 003900.66 & 482057.39 & 19.38 & 17.27 & 193 & 0.43 & 5.4 & - & - & $\mathrm{S}$ & 09401324 \\
\hline 185V000097 & 003900.68 & 482136.07 & 20.17 & - & 121 & 0.99 & 4.9 & - & - & $\mathrm{S}$ & 09411530 \\
\hline 185V000098 & 003900.53 & 481916.75 & 20.07 & - & 127 & 0.73 & 3.9 & - & - & $\mathrm{S}$ & 09420788 \\
\hline 185V000099 & 003900.53 & 481927.13 & 20.65 & 17.43 & 287 & 1.09 & 5.4 & - & - & $\mathrm{C}$ & 09420843 \\
\hline $185 \mathrm{~V} 000100$ & 003900.58 & 482026.85 & 19.23 & 15.22 & 494 & 1.41 & 6.5 & - & - & $\mathrm{S}$ & 09431162 \\
\hline 185V000101 & 003900.42 & 481800.55 & 20.78 & - & 133 & 1.40 & 5.9 & - & - & $\mathrm{S}$ & 09430382 \\
\hline 185V000102 & 003900.57 & 482052.10 & 19.65 & 17.79 & 121 & 0.54 & 4.1 & - & - & $\mathrm{S}$ & 09451296 \\
\hline $185 \mathrm{~V} 000103$ & 003900.57 & 482120.26 & 19.25 & 16.93 & 224 & 0.64 & 5.2 & - & - & M & 09461446 \\
\hline $185 \mathrm{~V} 000104$ & 003900.36 & 482032.63 & 18.96 & 15.81 & 302 & 0.61 & 5.8 & - & - & $\mathrm{S}$ & 09551192 \\
\hline 185V000105 & 003900.10 & 482028.07 & 19.46 & 16.70 & 230 & 0.66 & 5.5 & - & - & $\mathrm{S}$ & 09681168 \\
\hline 185V000106 & 003900.03 & 482219.33 & 19.71 & 16.10 & 330 & 0.4 & 5.6 & - & - & $\mathrm{S}$ & 09781761 \\
\hline 185V000107 & 003859.62 & 481737.61 & 20.62 & 17.40 & 234 & 0.67 & 4.6 & - & - & $\mathrm{C}$ & 09850259 \\
\hline 185V000108 & 003859.86 & 482147.85 & 19.50 & 17.24 & 192 & 0.63 & 5.9 & - & - & M & 09851593 \\
\hline 185V000109 & 003859.73 & 482011.69 & 20.28 & 17.25 & 206 & 1.03 & 2.8 & - & - & M & 09871081 \\
\hline $185 \mathrm{~V} 000110$ & 003859.67 & 481947.12 & 20.16 & 16.51 & 306 & 0.92 & 6.3 & - & - & $\mathrm{C}$ & 09890950 \\
\hline & 003859.54 & & & 16.74 & 140 & 1.09 & 6.5 & - & - & M & 09960945 \\
\hline 185V000112 & 003859.58 & 482039.46 & 19.33 & 17.34 & 111 & 0.26 & 3.3 & - & - & $\mathrm{S}$ & 09961228 \\
\hline $185 \mathrm{~V} 000113$ & 003859.55 & 482058.61 & 19.29 & 16.56 & 248 & 0.88 & 5.7 & - & - & M & 09991330 \\
\hline 185V000114 & 003859.50 & 482043.15 & 19.39 & 16.94 & 167 & 0.57 & 3.9 & - & - & $\mathrm{C}$ & 10011248 \\
\hline 185V000115 & 003859.39 & 482147.63 & 19.04 & 17.51 & 165 & 0.32 & 6.2 & - & - & $\mathrm{S}$ & 10101592 \\
\hline 185V000116 & 003859.27 & & 19.63 & 17.64 & 172 & 0.59 & 5.0 & - & - & $\mathrm{M}$ & 10121081 \\
\hline 185V000117 & 003859.21 & 482057.33 & 19.67 & 16.27 & 358 & 0.41 & 5.7 & - & - & $\mathrm{C}$ & 10171324 \\
\hline 185V000118 & 003859.28 & 482254.66 & 20.09 & 17.03 & 103 & 0.50 & 3.2 & - & - & $\mathrm{S}$ & 10191949 \\
\hline 185V000119 & 003858.97 & 481926.65 & 19.07 & 16.89 & 137 & 0.30 & 3.2 & - & - & M & 10250840 \\
\hline
\end{tabular}


Table A.2. continued.

\begin{tabular}{|c|c|c|c|c|c|c|c|c|c|c|c|}
\hline ID & $\begin{array}{c}\text { RAJ2000 } \\
\text { h:m:s }\end{array}$ & $\begin{array}{c}\text { DEJ2000 } \\
\text { d:m:s }\end{array}$ & $\begin{array}{c}i \\
{[\mathrm{mag}]}\end{array}$ & $\begin{array}{c}K_{\mathrm{s}} \\
{[\mathrm{mag}]}\end{array}$ & $\begin{array}{l}P_{0} \\
{[\mathrm{~d}]}\end{array}$ & $\begin{array}{c}\Delta i \\
{[\mathrm{mag}]}\end{array}$ & $\operatorname{sig}_{0}$ & $\begin{array}{l}P_{1} \\
{[\mathrm{~d}]}\end{array}$ & $\operatorname{sig}_{1}$ & type & ID Paper I \\
\hline $185 \mathrm{~V} 000120$ & 003859.03 & 482142.05 & 19.44 & 16.52 & 314 & 0.63 & 6.6 & - & - & $\mathrm{C}$ & 10291562 \\
\hline 185V000121 & 003858.78 & 481734.79 & 20.65 & 17.55 & 199 & 1.10 & 4.8 & - & - & $\mathrm{u}$ & - \\
\hline $185 \mathrm{~V} 000122$ & 003858.97 & 482115.09 & 19.68 & 17.15 & 273 & 0.82 & 6.9 & - & - & $\mathrm{C}$ & 10311418 \\
\hline $185 \mathrm{~V} 000123$ & 003858.67 & 481956.06 & 19.88 & 17.17 & 116 & 0.65 & 3.7 & - & - & M & 10420997 \\
\hline $185 \mathrm{~V} 000124$ & 003858.49 & 482115.74 & 19.88 & 16.93 & 217 & 1.15 & 6.7 & - & - & M & 10561421 \\
\hline $185 \mathrm{~V} 000125$ & 003858.30 & 481922.84 & 19.65 & 17.60 & 192 & 0.71 & 6.0 & - & - & M & 10600820 \\
\hline $185 \mathrm{~V} 000126$ & 003858.17 & 481806.23 & 19.97 & 17.22 & 202 & 0.58 & 5.6 & - & - & $\mathrm{M}$ & 10640411 \\
\hline $185 \mathrm{~V} 000127$ & 003858.22 & 481904.48 & 20.53 & - & 104 & 0.69 & 3.0 & - & - & $\mathrm{S}$ & 10640722 \\
\hline $185 \mathrm{~V} 000128$ & 003858.18 & 481937.05 & 20.01 & 17.56 & 511 & 0.34 & 4.0 & - & - & M & 10670895 \\
\hline $185 \mathrm{~V} 000129$ & 003858.14 & 481930.85 & 19.27 & 17.09 & 208 & 0.39 & 6.2 & - & - & $\mathrm{S}$ & 10690862 \\
\hline $185 \mathrm{~V} 000130$ & 003858.04 & 481859.95 & 19.88 & 17.07 & 253 & 0.52 & 5.1 & - & - & $\mathrm{C}$ & 10730698 \\
\hline 185V000131 & 003858.10 & 482007.57 & 18.69 & 16.01 & 232 & 0.50 & 4.9 & - & - & $\mathrm{S}$ & 10731058 \\
\hline $185 \mathrm{~V} 000132$ & 003858.14 & 482048.40 & 19.46 & 16.23 & 277 & 0.35 & 4.9 & - & - & M & 10731276 \\
\hline $185 \mathrm{~V} 000133$ & 003858.02 & 481939.68 & 19.24 & 17.06 & 155 & 0.43 & 5.8 & - & - & M & 10760909 \\
\hline 185V000134 & 003858.05 & 482059.64 & 20.11 & 17.82 & 223 & 1.45 & 6.7 & - & - & $\mathrm{S}$ & 10781336 \\
\hline $185 \mathrm{~V} 000135$ & 003857.95 & 481941.21 & 20.10 & 16.91 & 240 & 1.30 & 5.8 & - & - & M & 10800917 \\
\hline $185 \mathrm{~V} 000136$ & 003857.73 & 482209.78 & 19.96 & - & 119 & 0.50 & 3.7 & - & - & $\mathrm{S}$ & 10991709 \\
\hline $185 \mathrm{~V} 000137$ & 003857.53 & 482009.41 & 19.00 & 16.70 & 157 & 0.41 & 3.6 & - & - & M & 11041068 \\
\hline 185V000138 & 003857.51 & 482137.74 & 19.38 & 17.13 & 168 & 0.59 & 4.2 & - & - & $\mathrm{S}$ & 11091538 \\
\hline 185V000139 & 003842.59 & 482141.79 & 20.33 & 16.37 & 323 & 0.88 & 4.6 & - & - & $\mathrm{u}$ & 19001556 \\
\hline $185 \mathrm{~V} 000140$ & 003857.35 & 481856.01 & 19.53 & 17.40 & 199 & 0.39 & 6.0 & - & - & S & 11100676 \\
\hline $185 \mathrm{~V} 000141$ & 003857.26 & 481735.11 & 19.94 & - & 130 & 0.81 & 4.7 & - & - & $\mathrm{S}$ & 11100245 \\
\hline 185V000142 & 003842.56 & 482123.55 & 19.92 & - & 185 & 0.56 & 6.0 & - & - & M & 19011458 \\
\hline $185 \mathrm{~V} 000143$ & 003857.07 & 481933.09 & 19.56 & 16.94 & 246 & 0.50 & 5.2 & - & - & $\mathrm{C}$ & 11260874 \\
\hline $185 \mathrm{~V} 000144$ & 003856.91 & 481907.43 & 20.64 & 17.44 & 206 & 0.64 & 5.2 & - & - & $\mathrm{u}$ & 11330737 \\
\hline $185 \mathrm{~V} 000145$ & 003842.52 & 481917.57 & 19.83 & 17.84 & 134 & 0.48 & 4.8 & - & - & M & 18980786 \\
\hline $185 \mathrm{~V} 000146$ & 003857.03 & 482151.90 & 19.86 & 17.43 & 175 & 0.69 & 5.9 & - & - & M & 11351614 \\
\hline $185 \mathrm{~V} 000147$ & 003856.75 & 482048.19 & 20.18 & 17.15 & 211 & 0.45 & 3.7 & - & - & M & 11471274 \\
\hline $185 \mathrm{~V} 000148$ & 003856.69 & 482040.91 & 20.12 & 15.94 & 343 & 0.72 & 4.6 & - & - & $\mathrm{S}$ & 11501235 \\
\hline 185V000149 & 003856.84 & 482259.45 & 19.62 & 17.38 & 209 & 0.44 & 5.8 & - & - & M & 11491974 \\
\hline $185 \mathrm{~V} 000150$ & 003856.51 & 481859.92 & 20.33 & 16.81 & 231 & 1.59 & 6.2 & - & - & M & 11540697 \\
\hline 185V000151 & 003856.16 & 481825.41 & 20.25 & - & 166 & 1.41 & 5.8 & - & - & $\mathrm{S}$ & 11710513 \\
\hline 185V000152 & 003856.01 & 481812.58 & 20.17 & 17.68 & 150 & 0.85 & 6.4 & - & - & M & 11780444 \\
\hline $185 \mathrm{~V} 000153$ & 003856.13 & 482033.95 & 18.83 & 17.03 & 178 & 0.40 & 4.9 & - & - & $\mathrm{S}$ & 11791198 \\
\hline $185 \mathrm{~V} 000154$ & 003856.03 & 481919.52 & 20.85 & - & 132 & 1.73 & 6.2 & - & - & M & 11810801 \\
\hline $185 \mathrm{~V} 000155$ & 003856.01 & 481956.10 & 19.93 & 16.37 & 406 & 1.23 & 6.1 & - & - & $\mathrm{S}$ & 11840996 \\
\hline 185V000156 & 003856.09 & 482053.50 & 19.26 & 16.94 & 217 & 0.68 & 6.5 & - & - & M & 11821302 \\
\hline $185 \mathrm{~V} 000157$ & 003855.84 & 481906.82 & 19.84 & 17.10 & 112 & 0.32 & 4.9 & - & - & M & 11900733 \\
\hline 185V000158 & 003855.99 & 482238.72 & 19.86 & 17.91 & 150 & 0.76 & 5.6 & - & - & $\mathrm{S}$ & 11931863 \\
\hline $185 \mathrm{~V} 000159$ & 003855.79 & 481926.22 & 19.72 & 17.37 & 182 & 1.06 & 6.8 & - & - & M & 11940837 \\
\hline $185 \mathrm{~V} 000160$ & 003842.54 & 481837.26 & 20.94 & 16.62 & 361 & 0.99 & 3.8 & - & - & $\mathrm{C}$ & 18950571 \\
\hline $185 \mathrm{~V} 000161$ & 003855.77 & 481915.06 & 20.08 & - & 114 & 0.56 & 5.7 & - & - & $\mathrm{S}$ & 11940777 \\
\hline $185 \mathrm{~V} 000162$ & 003855.77 & 482050.61 & 20.14 & - & 116 & 0.66 & 4.0 & - & - & $\mathrm{S}$ & 11991287 \\
\hline $185 \mathrm{~V} 000163$ & 003855.72 & 482140.91 & 19.55 & 16.68 & 285 & 0.50 & 6.1 & - & - & $\mathrm{u}$ & - \\
\hline $185 \mathrm{~V} 000164$ & 003855.47 & 482035.56 & 19.26 & 15.91 & 453 & 0.74 & 4.6 & - & - & $\mathrm{S}$ & 12141206 \\
\hline 185V000165 & 003855.25 & 482056.07 & 19.98 & 16.39 & 370 & 1.67 & 6.9 & - & - & M & 12271316 \\
\hline $185 \mathrm{~V} 000166$ & 003855.19 & 482018.10 & 19.94 & 16.41 & 361 & 0.74 & 3.0 & - & - & M & 12281113 \\
\hline $185 \mathrm{~V} 000167$ & 003855.10 & 481915.63 & 19.96 & 17.15 & 107 & 0.50 & 3.7 & - & - & M & 12300780 \\
\hline $185 \mathrm{~V} 000168$ & 003855.14 & 482135.46 & 19.85 & 16.41 & 308 & 0.69 & 6.3 & - & - & $\mathrm{S}$ & 12351525 \\
\hline $185 \mathrm{~V} 000169$ & 003855.08 & 482059.78 & 19.32 & 15.92 & 402 & 0.54 & 6.0 & - & - & $\mathrm{S}$ & 12361335 \\
\hline $185 \mathrm{~V} 000170$ & 003855.03 & 482005.16 & 20.34 & 16.83 & 272 & 1.35 & 6.2 & - & - & $\mathrm{u}$ & - \\
\hline 185V000171 & 003855.05 & 482047.04 & 19.16 & 17.03 & 91 & 0.26 & 2.7 & - & - & $\mathrm{S}$ & 12371267 \\
\hline $185 \mathrm{~V} 000172$ & 003855.04 & 482121.21 & 19.81 & - & 176 & 0.76 & 6.3 & - & - & M & 12391450 \\
\hline $185 \mathrm{~V} 000173$ & 003855.02 & 482142.04 & 20.32 & 16.67 & 287 & 0.93 & 5.6 & - & - & $\mathrm{C}$ & 12411561 \\
\hline $185 \mathrm{~V} 000174$ & 003842.54 & 481827.86 & 19.80 & 17.56 & 160 & 0.56 & 6.1 & - & - & M & 18950520 \\
\hline $185 \mathrm{~V} 000175$ & 003854.81 & 481933.64 & 19.82 & 17.61 & 153 & 0.74 & 5.6 & - & - & M & 12460876 \\
\hline $185 \mathrm{~V} 000176$ & 003854.59 & 481906.97 & 19.64 & 16.26 & 394 & 0.44 & 5.4 & - & - & $\mathrm{C}$ & 12570734 \\
\hline $185 \mathrm{~V} 000177$ & 003854.23 & 481724.52 & 19.71 & 17.45 & 170 & 1.02 & 5.9 & - & - & M & 12710187 \\
\hline $185 \mathrm{~V} 000178$ & 003854.38 & 482015.87 & 19.78 & 16.63 & 280 & 0.83 & 6.2 & - & - & $\mathrm{C}$ & 12711101 \\
\hline $185 \mathrm{~V} 000179$ & 003854.31 & 482114.94 & 19.83 & 16.93 & 296 & 1.01 & 6.1 & - & - & $\mathrm{u}$ & - \\
\hline
\end{tabular}


Table A.2. continued.

\begin{tabular}{|c|c|c|c|c|c|c|c|c|c|c|c|}
\hline ID & $\begin{array}{c}\text { RAJ2000 } \\
\text { h:m:s }\end{array}$ & $\begin{array}{c}\text { DEJ2000 } \\
\text { d:m:s }\end{array}$ & $\begin{array}{c}i \\
{[\mathrm{mag}]}\end{array}$ & $\begin{array}{c}K_{\mathrm{s}} \\
{[\mathrm{mag}]}\end{array}$ & $\begin{array}{l}P_{0} \\
{[\mathrm{~d}]}\end{array}$ & $\begin{array}{c}\Delta i \\
{[\mathrm{mag}]}\end{array}$ & $s i g_{0}$ & $\begin{array}{l}P_{1} \\
\text { [d] }\end{array}$ & $s i g_{1}$ & type & ID ${ }_{\text {Paper I }}$ \\
\hline $185 \mathrm{~V} 000180$ & 003854.06 & $48 \quad 1919.72$ & 19.68 & 17.50 & 187 & 0.64 & 6.4 & - & - & $\bar{M}$ & 12850801 \\
\hline 185V000181 & 003854.01 & 481858.37 & 19.62 & 17.82 & 154 & 0.53 & 5.8 & - & - & M & 12870688 \\
\hline $185 \mathrm{~V} 000182$ & 003853.77 & 482039.45 & 19.57 & 16.54 & 739 & 0.33 & 3.5 & - & - & $\mathrm{u}$ & 13041226 \\
\hline $185 \mathrm{~V} 000183$ & 003853.44 & 481752.39 & 19.54 & 16.25 & 392 & 1.52 & 6.5 & - & - & $\mathrm{u}$ & 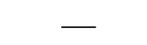 \\
\hline 185V000184 & 003853.43 & 481754.60 & 19.04 & 16.83 & 263 & 0.37 & 6.4 & - & - & $\mathrm{S}$ & 13150347 \\
\hline 185V000185 & 003853.34 & 481858.91 & 20.06 & 17.76 & 141 & 0.75 & 5.6 & - & - & M & 13230690 \\
\hline 185V000186 & 003853.29 & 482000.66 & 19.39 & 17.25 & 112 & 0.20 & 4.5 & - & - & M & 13281019 \\
\hline 185V000187 & 003853.25 & 482122.94 & 20.27 & - & 97 & 0.46 & 3.4 & - & - & $\mathrm{S}$ & 13341458 \\
\hline 185V000188 & 003853.13 & 481942.43 & 20.41 & 16.39 & 303 & 1.01 & 5.6 & - & - & M & 13360922 \\
\hline 185V000189 & 003853.24 & 482221.16 & 20.18 & - & 92 & 0.46 & 4.5 & - & - & $\mathrm{S}$ & 13371769 \\
\hline $185 \mathrm{~V} 000190$ & 003852.86 & 481811.09 & 19.62 & 16.44 & 300 & 0.41 & 6.1 & - & - & $\mathrm{C}$ & 13460435 \\
\hline 185V000191 & 003852.84 & 481914.08 & 19.83 & - & 94 & 0.47 & 3.3 & - & - & $\mathrm{S}$ & 13500771 \\
\hline 185V000192 & 003852.85 & 481920.93 & 19.95 & - & 154 & 0.61 & 5.0 & - & - & M & 13500807 \\
\hline $185 \mathrm{~V} 000193$ & 003852.43 & 481950.16 & 18.90 & - & 397 & 0.25 & 4.2 & - & - & $\mathrm{C}$ & 13740963 \\
\hline 185V000194 & 003852.49 & 482257.80 & 20.22 & 17.81 & 172 & 1.21 & 4.7 & - & - & $\mathrm{S}$ & 13791964 \\
\hline 185V000195 & 003852.35 & 482014.19 & 19.19 & 17.16 & 115 & 0.22 & 2.6 & - & - & M & 13791091 \\
\hline 185V000196 & 003852.09 & 481808.68 & 20.34 & 17.76 & 236 & 1.04 & 5.5 & - & - & $\mathrm{C}$ & 13860422 \\
\hline 185V000197 & 003852.08 & 481915.15 & 19.31 & 16.97 & 241 & 0.45 & 4.8 & - & - & M & 13900776 \\
\hline 185V000198 & 003852.06 & 481958.40 & 19.53 & 16.17 & 257 & 0.68 & 6.4 & - & - & $\mathrm{S}$ & 13931007 \\
\hline 185V000199 & 003851.78 & 482017.38 & 19.57 & 17.01 & 180 & 0.67 & 5.7 & - & - & $\mathrm{S}$ & 14091108 \\
\hline $185 \mathrm{~V} 000200$ & 003851.42 & 481814.65 & 19.93 & 16.88 & 285 & 0.83 & 6.0 & - & - & $\mathrm{C}$ & 14220453 \\
\hline 185V000201 & 003851.40 & 482058.43 & 19.49 & 16.76 & 206 & 0.75 & 6.1 & - & - & $\mathrm{S}$ & 14311327 \\
\hline $185 \mathrm{~V} 000202$ & 003851.34 & 482019.89 & 20.11 & 17.01 & 289 & 1.28 & 5.0 & - & - & $\mathrm{u}$ & 14321121 \\
\hline $185 \mathrm{~V} 000203$ & 003851.04 & 482036.08 & 19.38 & 17.41 & 168 & 0.31 & 4.0 & - & - & M & 14491208 \\
\hline $185 \mathrm{~V} 000204$ & 003850.65 & 481817.71 & 19.71 & 17.15 & 122 & 0.36 & 4.6 & - & - & M & 14640469 \\
\hline $185 \mathrm{~V} 000205$ & 003850.47 & 482023.29 & 19.52 & 17.22 & 200 & 0.64 & 4.6 & - & - & $\mathrm{S}$ & 14791139 \\
\hline $185 \mathrm{~V} 000206$ & 003850.31 & 481736.98 & 19.19 & 17.18 & 205 & 0.40 & 6.8 & - & - & M & 14800252 \\
\hline 185V000207 & 003850.36 & 481853.58 & 19.67 & 17.67 & 208 & 0.45 & 4.6 & - & - & M & 14810661 \\
\hline 185V000208 & 003850.45 & 482052.35 & 19.39 & 17.02 & 152 & 0.58 & 4.8 & - & - & M & 14811294 \\
\hline $185 \mathrm{~V} 000209$ & 003850.24 & 481932.47 & 19.82 & 17.82 & 161 & 0.37 & 4.2 & - & - & M & 14890868 \\
\hline $185 \mathrm{~V} 000210$ & 003849.94 & 481739.17 & 19.83 & - & 119 & 0.32 & 3.8 & - & - & $\mathrm{S}$ & 14990263 \\
\hline $185 \mathrm{~V} 000211$ & 003850.03 & 482215.85 & 19.87 & 17.32 & 158 & 1.01 & 5.6 & - & - & $\mathrm{S}$ & 15071739 \\
\hline $185 \mathrm{~V} 000212$ & 003849.93 & 482034.82 & 18.61 & 16.43 & 312 & 0.79 & 6.4 & - & - & $\mathrm{S}$ & 15081200 \\
\hline $185 \mathrm{~V} 000213$ & 003849.84 & 482105.87 & 19.56 & 17.42 & 220 & 0.77 & 6.4 & - & - & $\mathrm{S}$ & 15151366 \\
\hline $185 \mathrm{~V} 000214$ & 003849.43 & 481827.55 & 19.73 & 18.21 & 171 & 0.62 & 5.8 & - & - & $\mathrm{S}$ & 15290521 \\
\hline $185 \mathrm{~V} 000215$ & 003849.37 & 481928.71 & 19.59 & 17.16 & 188 & 0.39 & 4.8 & - & - & M & 15340848 \\
\hline 185V000216 & 003849.41 & 482015.68 & 19.61 & 17.56 & 205 & 0.63 & 4.9 & - & - & M & 15341098 \\
\hline $185 \mathrm{~V} 000217$ & 003849.10 & 481728.60 & 19.75 & 17.82 & 160 & 0.45 & 5.8 & - & - & M & 15430207 \\
\hline $185 \mathrm{~V} 000218$ & 003849.02 & 481815.42 & 19.59 & 17.64 & 190 & 0.57 & 6.1 & - & - & $\mathrm{S}$ & 15500456 \\
\hline $185 \mathrm{~V} 000219$ & 003848.86 & 482105.05 & 19.43 & 17.17 & 112 & 0.39 & 3.9 & - & - & M & 15661361 \\
\hline $185 \mathrm{~V} 000220$ & 003848.63 & 481834.52 & 19.56 & - & 133 & 0.32 & 4.8 & - & - & M & 15710558 \\
\hline $185 \mathrm{~V} 000221$ & 003848.60 & 482052.10 & 18.91 & 16.14 & 303 & 0.25 & 5.0 & - & - & $\mathrm{C}$ & 15791292 \\
\hline $185 \mathrm{~V} 000222$ & 003848.49 & 481913.87 & 19.67 & 17.49 & 92 & 0.22 & 2.1 & - & - & M & 15810768 \\
\hline $185 \mathrm{~V} 000223$ & 003848.30 & 481734.86 & 19.82 & 17.75 & 164 & 0.86 & 5.1 & - & - & M & 15860240 \\
\hline $185 \mathrm{~V} 000224$ & 003848.18 & 482004.06 & 19.75 & 16.91 & 187 & 0.35 & 4.5 & - & - & M & 15991036 \\
\hline $185 \mathrm{~V} 000225$ & 003848.11 & & 20.24 & 16.89 & 313 & 0.95 & 5.8 & - & - & $\mathrm{S}$ & 16031072 \\
\hline $185 \mathrm{~V} 000226$ & 003847.96 & 481956.27 & 19.69 & 17.76 & 158 & 0.46 & 5.1 & - & - & M & 16110994 \\
\hline $185 \mathrm{~V} 000227$ & 003847.75 & 482023.68 & 19.72 & 17.84 & 123 & 0.70 & 5.1 & - & - & $\mathrm{S}$ & 16231140 \\
\hline $185 \mathrm{~V} 000228$ & 003847.65 & 482136.64 & 19.51 & - & 100 & 0.34 & 4.6 & - & - & $\mathrm{S}$ & 16321530 \\
\hline $185 \mathrm{~V} 000229$ & 003847.38 & 482047.94 & 20.17 & - & 167 & 0.93 & 6.1 & - & - & $\mathrm{S}$ & 16441270 \\
\hline $185 \mathrm{~V} 000230$ & 003842.88 & 482049.54 & 19.74 & 16.98 & 222 & 0.58 & 4.9 & - & - & M & 18831277 \\
\hline $185 \mathrm{~V} 000231$ & 003847.08 & 481924.84 & 19.86 & 17.70 & 207 & 1.20 & 6.0 & - & - & M & 16560826 \\
\hline $185 \mathrm{~V} 000232$ & 003846.93 & 481850.94 & 19.96 & 17.84 & 193 & 0.78 & 6.8 & - & - & M & 16620645 \\
\hline $185 \mathrm{~V} 000233$ & 003846.95 & & 19.91 & 17.71 & 141 & 0.84 & 5.4 & - & - & $\mathrm{S}$ & 16711714 \\
\hline $185 \mathrm{~V} 000234$ & 003846.67 & 481715.30 & 19.70 & - & 191 & 1.04 & 5.1 & - & - & $\mathrm{u}$ & 16720135 \\
\hline $185 \mathrm{~V} 000235$ & 003843.03 & 482004.03 & 19.15 & 16.89 & 211 & 0.44 & 5.9 & - & - & $\mathrm{S}$ & 18731034 \\
\hline $185 \mathrm{~V} 000236$ & 003846.61 & 482103.26 & 19.70 & - & 185 & 0.37 & 3.8 & - & - & $\mathrm{M}$ & 16851351 \\
\hline $185 \mathrm{~V} 000237$ & 003846.56 & 482030.31 & 20.03 & 17.84 & 131 & 0.41 & 3.7 & - & - & $\mathrm{S}$ & 16871175 \\
\hline & 003846.21 & 481829.68 & 20.26 & 17.19 & 393 & 1.10 & 6.0 & - & - & $\mathrm{u}$ & 17000531 \\
\hline $185 \mathrm{~V} 000239$ & 003846.33 & 482240.02 & 19.29 & 17.61 & 160 & 0.62 & 5.3 & - & - & M & 17051867 \\
\hline
\end{tabular}


Table A.2. continued.

\begin{tabular}{|c|c|c|c|c|c|c|c|c|c|c|c|}
\hline ID & $\begin{array}{c}\text { RAJ2000 } \\
\text { h:m:s }\end{array}$ & $\begin{array}{l}\text { DEJ2000 } \\
\text { d:m:s }\end{array}$ & $\begin{array}{c}i \\
{[\mathrm{mag}]}\end{array}$ & $\begin{array}{c}K_{\mathrm{s}} \\
{[\mathrm{mag}]}\end{array}$ & $\begin{array}{l}P_{0} \\
{[\mathrm{~d}]}\end{array}$ & $\begin{array}{c}\Delta i \\
{[\mathrm{mag}]}\end{array}$ & $\operatorname{sig}_{0}$ & $\begin{array}{l}P_{1} \\
{[\mathrm{~d}]}\end{array}$ & $\operatorname{sig}_{1}$ & type & ID $D_{\text {Paper I }}$ \\
\hline $185 \mathrm{~V} 000240$ & 003843.00 & 481806.39 & 19.36 & 17.14 & 238 & 0.99 & 5.3 & - & - & $\bar{M}$ & 18690406 \\
\hline $185 \mathrm{~V} 000241$ & 003845.99 & 481831.54 & 19.33 & 17.89 & 206 & 0.57 & 5.9 & - & - & $\mathrm{S}$ & 17120541 \\
\hline $185 \mathrm{~V} 000242$ & 003845.92 & 481901.99 & 19.00 & 16.11 & 303 & 1.28 & 6.3 & - & - & M & 17160704 \\
\hline $185 \mathrm{~V} 000243$ & 003845.86 & 481832.29 & 19.58 & 17.15 & 259 & 1.65 & 6.6 & - & - & M & 17180545 \\
\hline $185 \mathrm{~V} 000244$ & 003843.25 & 482028.08 & 19.58 & 16.68 & 249 & 0.62 & 6.3 & - & - & $\mathrm{C}$ & 18621162 \\
\hline $185 \mathrm{~V} 000245$ & 003843.58 & 481930.92 & 19.99 & 17.35 & 210 & 0.90 & 5.8 & - & - & $\mathrm{S}$ & 18420857 \\
\hline $185 \mathrm{~V} 000246$ & 003845.68 & 482206.22 & 19.80 & 17.38 & 165 & 0.39 & 3.9 & - & - & $\mathrm{S}$ & 17381687 \\
\hline $185 \mathrm{~V} 000247$ & 003843.63 & 481918.87 & 19.74 & 17.57 & 161 & 0.52 & 5.5 & - & - & M & 18390793 \\
\hline $185 \mathrm{~V} 000248$ & 003845.54 & 482118.22 & 19.58 & 16.68 & 279 & 0.53 & 4.3 & - & - & M & 17431431 \\
\hline $185 \mathrm{~V} 000249$ & 003845.36 & 481957.27 & 20.38 & 16.97 & 231 & 0.96 & 6.4 & - & - & $\mathrm{u}$ & 17490999 \\
\hline $185 \mathrm{~V} 000250$ & 003843.60 & 481728.18 & 19.75 & 17.26 & 206 & 0.48 & 3.9 & - & - & $\mathrm{u}$ & 18360202 \\
\hline 185V000251 & 003845.33 & 482035.60 & 19.77 & 17.21 & 187 & 0.29 & 4.0 & - & - & $\mathrm{C}$ & 17521203 \\
\hline $185 \mathrm{~V} 000252$ & 003845.14 & 482047.11 & 20.41 & 16.68 & 229 & 0.91 & 6.4 & - & - & $\mathrm{C}$ & 17631265 \\
\hline $185 \mathrm{~V} 000253$ & 003844.80 & 482054.68 & 19.44 & 17.11 & 163 & 0.36 & 5.6 & - & - & M & 17811305 \\
\hline $185 \mathrm{~V} 000254$ & 003844.34 & 481918.45 & 20.06 & - & 117 & 0.51 & 5.0 & - & - & $\mathrm{S}$ & 18010791 \\
\hline $185 \mathrm{~V} 000255$ & 003844.27 & 482304.59 & 20.59 & - & 104 & 0.74 & 3.9 & - & - & $\mathrm{u}$ & 18151998 \\
\hline $185 \mathrm{~V} 000256$ & 003844.13 & 482117.99 & 19.75 & - & 140 & 0.36 & 4.1 & - & - & S & 18181429 \\
\hline $185 \mathrm{~V} 000257$ & 003843.69 & 481736.07 & 20.04 & - & 129 & 0.59 & 4.7 & - & - & $\mathrm{S}$ & 18310244 \\
\hline $185 \mathrm{~V} 000258$ & 003844.05 & 482002.83 & 19.80 & 17.78 & 172 & 0.70 & 6.5 & - & - & M & 18191028 \\
\hline $185 \mathrm{~V} 000259$ & 003907.40 & 481935.47 & 20.77 & 16.70 & 262 & 1.74 & 4.6 & - & - & M & 05780891 \\
\hline $185 \mathrm{~V} 000260$ & 003905.60 & 481920.89 & 21.08 & - & 634 & 1.87 & 2.5 & - & - & $\mathrm{u}$ & - \\
\hline $185 \mathrm{~V} 000261$ & 003901.11 & 482204.11 & 21.76 & 17.13 & 439 & 2.09 & 3.1 & - & - & $\mathrm{C}$ & 09201680 \\
\hline $185 \mathrm{~V} 000262$ & 003859.26 & 481939.42 & 20.45 & 17.50 & 188 & 1.13 & 5.5 & - & - & $\mathrm{S}$ & 10110908 \\
\hline $185 \mathrm{~V} 000263$ & 003859.20 & 481957.65 & 20.36 & 17.34 & 173 & 1.60 & 5.3 & - & - & $\mathrm{u}$ & - \\
\hline $185 \mathrm{~V} 000264$ & 003858.08 & 481949.24 & 20.91 & 16.70 & 146 & 0.76 & 2.0 & - & - & $\mathrm{u}$ & 10730960 \\
\hline $185 \mathrm{~V} 000265$ & 003856.89 & 481941.08 & 20.70 & 17.21 & 194 & 1.16 & 4.4 & - & - & $\mathrm{u}$ & 11360916 \\
\hline $185 \mathrm{~V} 000266$ & 003855.78 & 481918.83 & 20.55 & 16.35 & 291 & 1.50 & 4.9 & - & - & $\mathrm{u}$ & - \\
\hline $185 \mathrm{~V} 000267$ & 003855.67 & 482014.19 & 20.43 & 16.84 & 215 & 0.69 & 5.1 & - & - & $\mathrm{u}$ & - \\
\hline $185 \mathrm{~V} 000268$ & 003906.88 & 481811.59 & 19.55 & 17.39 & 212 & 1.00 & 6.4 & - & - & $\mathrm{u}$ & - \\
\hline $185 \mathrm{~V} 000269$ & 003904.82 & 482017.53 & 19.98 & 17.12 & 227 & 0.99 & 6.3 & - & - & $\mathrm{u}$ & - \\
\hline $185 \mathrm{~V} 000270$ & 003903.86 & 482155.50 & 19.56 & 17.06 & 216 & 0.47 & 3.2 & - & - & $\mathrm{u}$ & - \\
\hline $185 \mathrm{~V} 000271$ & 003903.32 & 481940.79 & 20.32 & - & 122 & 0.94 & 5.0 & - & - & $\mathrm{u}$ & - \\
\hline $185 \mathrm{~V} 000272$ & 003902.78 & 482029.63 & 19.73 & 16.50 & 149 & 0.53 & 5.4 & - & - & $\mathrm{u}$ & - \\
\hline $185 \mathrm{~V} 000273$ & 003902.17 & 482008.29 & 20.34 & 17.20 & 246 & 0.91 & 4.6 & - & - & $\mathrm{u}$ & - \\
\hline $185 \mathrm{~V} 000274$ & 003902.14 & 482211.45 & 20.17 & 16.85 & 226 & 1.53 & 6.6 & - & - & $\mathrm{u}$ & - \\
\hline $185 \mathrm{~V} 000275$ & 003901.49 & 482000.86 & 19.86 & 17.12 & 226 & 0.96 & 6.0 & - & - & $\mathrm{u}$ & - \\
\hline $185 \mathrm{~V} 000276$ & 003901.05 & 481959.55 & 20.42 & 16.30 & 163 & 0.76 & 4.9 & - & - & $\mathrm{u}$ & - \\
\hline $185 \mathrm{~V} 000277$ & 003900.71 & 481813.05 & 19.79 & 17.24 & 236 & 0.51 & 5.9 & - & - & $\mathrm{u}$ & - \\
\hline $185 \mathrm{~V} 000278$ & 003900.61 & 482015.23 & 20.49 & 17.31 & 253 & 1.54 & 2.9 & - & - & $\mathrm{u}$ & - \\
\hline $185 \mathrm{~V} 000279$ & 003900.32 & 482038.44 & 20.38 & 17.85 & 162 & 0.99 & 4.7 & - & - & $\mathrm{u}$ & - \\
\hline $185 \mathrm{~V} 000280$ & 003859.76 & 481846.22 & 19.83 & 17.38 & 192 & 0.70 & 5.7 & - & - & $\mathrm{u}$ & - \\
\hline $185 \mathrm{~V} 000281$ & 003859.41 & 481925.57 & 20.00 & 16.64 & 156 & 0.56 & 5.1 & - & - & $\mathrm{u}$ & - \\
\hline $185 \mathrm{~V} 000282$ & 003858.96 & 481857.15 & 19.85 & 18.00 & 162 & 0.59 & 5.2 & - & - & $\mathrm{u}$ & - \\
\hline $185 \mathrm{~V} 000283$ & 003858.91 & 481934.50 & 20.61 & 16.22 & 408 & 1.15 & 5.7 & - & - & $\mathrm{u}$ & - \\
\hline $185 \mathrm{~V} 000284$ & 003857.20 & 481909.35 & 19.67 & 17.57 & 167 & 0.57 & 5.6 & - & - & $\mathrm{u}$ & - \\
\hline $185 \mathrm{~V} 000285$ & 003856.52 & 482123.72 & 20.32 & 17.80 & 162 & 1.15 & 4.6 & - & - & $\mathrm{u}$ & - \\
\hline $185 \mathrm{~V} 000286$ & 003855.80 & 482057.65 & 19.78 & 16.89 & 258 & 0.90 & 6.4 & - & - & $\mathrm{u}$ & - \\
\hline $185 \mathrm{~V} 000287$ & 003855.61 & 481940.35 & 19.78 & 17.36 & 196 & 0.72 & 6.4 & - & - & $\mathrm{u}$ & - \\
\hline $185 \mathrm{~V} 000288$ & 003854.64 & 482050.72 & 19.75 & 16.83 & 236 & 0.56 & 5.1 & - & - & $\mathrm{u}$ & - \\
\hline $185 \mathrm{~V} 000289$ & 003854.48 & 481904.08 & 20.18 & 16.11 & 389 & 0.54 & 6.6 & - & - & $\mathrm{u}$ & - \\
\hline $185 \mathrm{~V} 000290$ & 003854.30 & 482042.92 & 19.99 & 16.21 & 371 & 0.96 & 5.6 & - & - & $\mathrm{u}$ & - \\
\hline 185V000291 & 003852.79 & 481917.12 & 20.30 & 17.77 & 160 & 0.81 & 5.6 & - & - & $\mathrm{u}$ & - \\
\hline $185 \mathrm{~V} 000292$ & 003852.72 & 481921.51 & 19.92 & 17.65 & 176 & 0.72 & 5.5 & - & - & $\mathrm{u}$ & - \\
\hline $185 \mathrm{~V} 000293$ & 003852.48 & 481853.38 & 19.60 & 16.56 & 354 & 0.64 & 6.7 & - & - & $\mathrm{u}$ & - \\
\hline $185 \mathrm{~V} 000294$ & 003852.35 & 481714.19 & 19.57 & - & 205 & 0.91 & 4.0 & - & - & $\mathrm{u}$ & - \\
\hline $185 \mathrm{~V} 000295$ & 003852.23 & 481705.67 & 19.56 & - & 251 & 0.95 & 4.4 & - & - & $\mathrm{u}$ & - \\
\hline $185 \mathrm{~V} 000296$ & 003850.70 & 482023.70 & 21.07 & 16.68 & 252 & 2.27 & 5.6 & - & - & $\mathrm{u}$ & - \\
\hline $185 \mathrm{~V} 000297$ & 003850.52 & 481844.91 & 20.22 & - & 184 & 1.80 & 5.7 & 一 & - & $\mathrm{u}$ & - \\
\hline $185 \mathrm{~V} 000298$ & 003849.13 & 482044.39 & 20.28 & 16.86 & 271 & 1.80 & 6.3 & - & - & $\mathrm{u}$ & - \\
\hline $185 \mathrm{~V} 000299$ & 003848.63 & 482234.45 & 19.57 & 17.19 & 151 & 0.38 & 5.0 & - & - & $\mathrm{u}$ & - \\
\hline
\end{tabular}


Table A.2. continued.

\begin{tabular}{|c|c|c|c|c|c|c|c|c|c|c|c|}
\hline ID & $\begin{array}{c}\text { RAJ2000 } \\
\text { h:m:s }\end{array}$ & $\begin{array}{c}\text { DEJ2000 } \\
\text { d:m:s }\end{array}$ & $\begin{array}{c}i \\
{[\mathrm{mag}]}\end{array}$ & $\left.\begin{array}{c}K_{\mathrm{s}} \\
{[\mathrm{mag}]}\end{array}\right]$ & $\begin{array}{l}P_{0} \\
{[\mathrm{~d}]}\end{array}$ & $\begin{array}{c}\Delta i \\
{[\mathrm{mag}]}\end{array}$ & $s i g_{0}$ & $\begin{array}{l}P_{1} \\
{[\mathrm{~d}]}\end{array}$ & $\operatorname{sig}_{1}$ & type & ID \\
\hline $185 \mathrm{~V} 000300$ & 003843.38 & 481921.84 & 19.70 & 17.43 & 189 & 0.68 & 5.6 & - & - & $\mathrm{u}$ & - \\
\hline 185V000301 & 003843.23 & 481912.78 & 19.64 & 17.23 & 193 & 1.00 & 5.3 & - & - & $\mathrm{u}$ & - \\
\hline $185 \mathrm{~V} 000302$ & 003859.61 & 481950.13 & 20.12 & 16.87 & 124 & 0.36 & 4.1 & - & - & $\mathrm{u}$ & - \\
\hline $185 \mathrm{~V} 000303$ & 003858.87 & 482022.17 & 20.58 & 17.35 & 113 & 0.91 & 3.7 & - & - & $\mathrm{u}$ & - \\
\hline $185 \mathrm{~V} 000304$ & 003858.44 & 482012.75 & 20.88 & 16.90 & 182 & 1.48 & 4.1 & - & - & $\mathrm{u}$ & - \\
\hline $185 \mathrm{~V} 000305$ & 003856.56 & 482022.39 & 21.44 & 17.02 & 170 & 2.13 & 4.9 & - & - & $\mathrm{u}$ & - \\
\hline 185V000306 & 003853.27 & 482008.36 & 19.75 & 16.37 & 416 & 0.63 & 6.1 & - & - & $\mathrm{u}$ & - \\
\hline 185V000307 & 003852.60 & 482058.65 & 20.56 & 17.63 & 201 & 1.21 & 5.8 & - & - & $\mathrm{u}$ & - \\
\hline 185V000308 & 003915.40 & 481925.31 & 19.58 & - & 169 & 0.81 & 4.4 & - & - & M & 01530840 \\
\hline 185V000309 & 003915.30 & 482016.03 & 19.45 & 17.28 & 175 & 0.56 & 6.0 & - & - & $\mathrm{S}$ & 01611110 \\
\hline $185 \mathrm{~V} 000310$ & 003914.86 & 481927.66 & 19.69 & - & 236 & 0.48 & 5.0 & - & - & M & 01820852 \\
\hline $185 \mathrm{~V} 000311$ & 003911.83 & 482137.69 & 20.06 & - & 56 & 0.51 & 5.6 & - & - & $\mathrm{S}$ & 03501543 \\
\hline $185 \mathrm{~V} 000312$ & 003911.18 & 482152.31 & 19.52 & 17.27 & 113 & 0.24 & 5.0 & - & - & S & 03851620 \\
\hline $185 \mathrm{~V} 000313$ & 003908.95 & 482118.59 & 21.69 & 15.93 & 519 & 1.78 & 5.0 & - & - & $\mathrm{u}$ & - \\
\hline $185 \mathrm{~V} 000314$ & 003909.01 & 482253.98 & 19.35 & 16.44 & 144 & 0.30 & 6.9 & - & - & $\mathrm{C}$ & 05041948 \\
\hline $185 \mathrm{~V} 000315$ & 003908.57 & 481739.76 & 20.43 & - & 220 & 0.84 & 7.7 & - & - & $\mathrm{u}$ & - \\
\hline $185 \mathrm{~V} 000316$ & 003907.74 & 481926.81 & 19.16 & 17.18 & 111 & 0.27 & 4.8 & - & - & $\mathrm{S}$ & 05600845 \\
\hline 185V000317 & 003907.39 & 482027.02 & 19.16 & 16.66 & 171 & 0.26 & 4.4 & - & - & $\mathrm{C}$ & 05811165 \\
\hline 185V000318 & 003905.46 & 482155.99 & 19.86 & - & 137 & 0.36 & 5.4 & - & - & $\mathrm{u}$ & - \\
\hline 185V000319 & 003904.98 & 482051.17 & 19.85 & 17.12 & 102 & 0.43 & 5.3 & - & - & M & 07111293 \\
\hline $185 \mathrm{~V} 000320$ & 003904.66 & 482056.25 & 20.09 & 16.72 & 127 & 0.54 & 4.3 & - & - & $\mathrm{u}$ & - \\
\hline $185 \mathrm{~V} 000321$ & 003904.50 & 482030.15 & 19.66 & - & 164 & 0.27 & 3.7 & - & - & M & 07351181 \\
\hline $185 \mathrm{~V} 000322$ & 003904.32 & 482113.31 & 20.13 & - & 130 & 0.48 & 3.9 & - & - & $\mathrm{S}$ & 07471410 \\
\hline $185 \mathrm{~V} 000323$ & 003903.93 & 481946.72 & 20.18 & 17.45 & 239 & 0.72 & 7.1 & - & - & $\mathrm{u}$ & - \\
\hline $185 \mathrm{~V} 000324$ & 003903.68 & 482240.07 & 19.75 & - & 128 & 0.32 & 3.5 & - & - & $\mathrm{S}$ & 07851872 \\
\hline $185 \mathrm{~V} 000325$ & 003903.36 & 482026.12 & 20.09 & 17.10 & 197 & 0.52 & 4.0 & - & - & $\mathrm{M}$ & 07951159 \\
\hline $185 \mathrm{~V} 000326$ & 003902.72 & 482016.13 & 20.23 & 17.23 & 101 & 0.47 & 4.6 & - & - & $\mathrm{u}$ & - \\
\hline $185 \mathrm{~V} 000327$ & 003901.72 & 481914.40 & 20.13 & 16.84 & 139 & 0.70 & 3.3 & - & - & M & 08780776 \\
\hline $185 \mathrm{~V} 000328$ & 003901.23 & 481709.18 & 19.76 & 17.58 & 212 & 0.66 & 6.7 & - & - & $\mathrm{u}$ & 08980109 \\
\hline $185 \mathrm{~V} 000329$ & 003901.47 & 482148.87 & 19.65 & - & 168 & 0.42 & 3.9 & - & - & $\mathrm{S}$ & 09001599 \\
\hline $185 \mathrm{~V} 000330$ & 003900.98 & 481726.66 & 19.87 & - & 128 & 0.60 & 4.5 & - & - & M & 09120202 \\
\hline $185 \mathrm{~V} 000331$ & 003900.77 & 481923.14 & 19.96 & 17.09 & 235 & 0.52 & 4.8 & - & - & M & 09300822 \\
\hline 185V000332 & 003900.81 & 482049.14 & 19.94 & - & 143 & 0.51 & 5.3 & - & - & $\mathrm{u}$ & - \\
\hline $185 \mathrm{~V} 000333$ & 003900.66 & 481939.05 & 19.84 & 16.79 & 113 & 0.35 & 4.2 & - & - & $\mathrm{u}$ & - \\
\hline $185 \mathrm{~V} 000334$ & 003900.45 & 481945.57 & 20.71 & - & 131 & 0.82 & 5.4 & - & - & M & 09480942 \\
\hline 185V000335 & 003859.83 & $48 \quad 1827.74$ & 19.68 & - & 160 & 0.43 & 5.5 & - & - & M & 09760527 \\
\hline $185 \mathrm{~V} 000336$ & 003859.65 & 481958.49 & 19.50 & - & 152 & 0.45 & 6.5 & - & - & $\mathrm{u}$ & - \\
\hline $185 \mathrm{~V} 000337$ & 003858.49 & 481757.21 & 19.76 & 17.25 & 196 & 0.35 & 3.8 & - & - & M & 10460363 \\
\hline 185V000338 & 003857.85 & 482019.06 & 18.94 & 15.29 & 200 & 0.30 & 4.9 & - & - & $\mathrm{u}$ & - \\
\hline $185 \mathrm{~V} 000339$ & 003857.72 & 481952.26 & 19.71 & 16.90 & 99 & 0.18 & 3.5 & - & - & M & 10930976 \\
\hline $185 \mathrm{~V} 000340$ & 003857.75 & 482043.55 & 19.90 & 16.24 & 890 & 0.50 & 7.1 & - & - & $\mathrm{u}$ & - \\
\hline $185 \mathrm{~V} 000341$ & 003857.18 & 481847.20 & 20.28 & 17.09 & 188 & 0.61 & 4.0 & - & - & $\mathrm{u}$ & - \\
\hline $185 \mathrm{~V} 000342$ & 003857.18 & 482029.45 & 22.09 & - & 175 & 1.61 & 3.0 & - & - & $\mathrm{u}$ & - \\
\hline $185 \mathrm{~V} 000343$ & 003857.05 & 482312.68 & 20.36 & 16.65 & 229 & 1.47 & 3.9 & - & - & $\mathrm{u}$ & - \\
\hline $185 \mathrm{~V} 000344$ & 003856.79 & 481949.73 & 19.37 & 16.60 & 266 & 0.40 & 6.0 & - & - & M & 11420962 \\
\hline $185 \mathrm{~V} 000345$ & 003856.71 & 481952.85 & 19.08 & 16.19 & 189 & 0.26 & 3.3 & - & - & $\mathrm{u}$ & - \\
\hline $185 \mathrm{~V} 000346$ & 003856.66 & 481957.68 & 19.51 & 16.78 & 809 & 0.30 & 5.0 & - & - & M & 11491005 \\
\hline $185 \mathrm{~V} 000347$ & 003856.59 & 481923.10 & 19.97 & 17.35 & 102 & 0.44 & 4.7 & - & - & $\mathrm{u}$ & - \\
\hline 185V000348 & 003856.46 & 481920.99 & 19.57 & 17.17 & 197 & 0.30 & 4.8 & - & - & $\mathrm{S}$ & 11580809 \\
\hline $185 \mathrm{~V} 000349$ & 003856.42 & 482144.64 & 20.16 & - & 154 & 0.36 & 3.2 & - & - & $\mathrm{S}$ & 11671575 \\
\hline $185 \mathrm{~V} 000350$ & 003856.43 & 482153.15 & 19.90 & 17.36 & 193 & 0.45 & 5.0 & - & - & $\mathrm{C}$ & 11671620 \\
\hline $185 \mathrm{~V} 000351$ & 003856.34 & & 20.12 & 16.24 & 270 & 0.97 & 6.6 & - & - & $\mathrm{u}$ & - \\
\hline $185 \mathrm{~V} 000352$ & 003856.28 & 482038.37 & 20.23 & 16.72 & 212 & 0.78 & 6.5 & - & - & $\mathrm{u}$ & - \\
\hline $185 \mathrm{~V} 000353$ & 003856.14 & 481925.17 & 19.21 & 16.08 & 195 & 0.27 & 6.4 & - & - & $\mathrm{C}$ & 11750831 \\
\hline $185 \mathrm{~V} 000354$ & 003856.03 & 481930.98 & 20.15 & 17.41 & 182 & 0.46 & 4.0 & - & - & $\mathrm{M}$ & 11810862 \\
\hline $185 \mathrm{~V} 000355$ & 003855.94 & 482038.78 & 19.14 & 16.36 & 159 & 0.25 & 4.4 & - & - & $\mathrm{C}$ & 11901224 \\
\hline $185 \mathrm{~V} 000356$ & 003855.36 & 482057.89 & 20.44 & 16.88 & 209 & 0.54 & 3.1 & - & - & $\mathrm{u}$ & - \\
\hline $185 \mathrm{~V} 000357$ & 003854.96 & 482026.88 & 19.58 & 17.17 & 110 & 0.32 & 4.6 & - & - & M & 12411160 \\
\hline $185 \mathrm{~V} 000358$ & 003854.56 & 481952.00 & 20.62 & - & 207 & 0.89 & 4.9 & - & - & $\mathrm{u}$ & 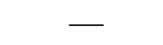 \\
\hline $185 \mathrm{~V} 000359$ & 003854.42 & 482248.90 & 19.65 & 18.78 & 105 & 0.15 & 4.1 & - & - & $\mathrm{S}$ & 12761917 \\
\hline
\end{tabular}


Table A.2. continued.

\begin{tabular}{|c|c|c|c|c|c|c|c|c|c|c|c|}
\hline ID & $\begin{array}{c}\text { RAJ2000 } \\
\text { h:m:s }\end{array}$ & $\begin{array}{c}\text { DEJ2000 } \\
\text { d:m:s }\end{array}$ & $\begin{array}{c}i \\
{[\mathrm{mag}]}\end{array}$ & $\begin{array}{c}K_{\mathrm{s}} \\
{[\mathrm{mag}]}\end{array}$ & $\begin{array}{l}P_{0} \\
{[d]}\end{array}$ & $\begin{array}{c}\Delta i \\
{[\mathrm{mag}]}\end{array}$ & $\operatorname{sig}_{0}$ & $\begin{array}{l}P_{1} \\
{[\mathrm{~d}]}\end{array}$ & $\operatorname{sig}_{1}$ & type & ID $D_{\text {Paper I }}$ \\
\hline $185 \mathrm{~V} 000360$ & 003854.21 & 482000.68 & 20.05 & - & 150 & 0.74 & 5.7 & - & - & $\mathrm{u}$ & - \\
\hline $185 \mathrm{~V} 000361$ & 003854.10 & 482057.57 & 20.21 & - & 170 & 0.53 & 5.2 & - & - & $\mathrm{u}$ & - \\
\hline 185V000362 & 003854.01 & 482001.80 & 20.43 & 16.81 & 123 & 0.51 & 3.8 & - & - & $\mathrm{u}$ & - \\
\hline $185 \mathrm{~V} 000363$ & 003853.86 & 481913.71 & 20.03 & - & 87 & 0.33 & 4.0 & - & - & $\mathrm{u}$ & - \\
\hline $185 \mathrm{~V} 000364$ & 003852.64 & 481859.56 & 20.51 & 17.80 & 108 & 0.72 & 3.9 & - & - & $\mathrm{u}$ & - \\
\hline $185 \mathrm{~V} 000365$ & 003852.67 & 482052.47 & 21.53 & - & 218 & 1.70 & 4.6 & - & - & $\mathrm{u}$ & - \\
\hline 185V000366 & 003852.01 & 481704.84 & 19.60 & - & 138 & 0.40 & 3.9 & - & - & $\mathrm{u}$ & - \\
\hline $185 \mathrm{~V} 000367$ & 003852.10 & 481935.18 & 19.61 & 17.46 & 188 & 0.26 & 6.5 & - & - & $\mathrm{u}$ & - \\
\hline $185 \mathrm{~V} 000368$ & 003851.93 & 481934.25 & 19.65 & - & 82 & 0.38 & 3.9 & - & - & S & 13990878 \\
\hline $185 \mathrm{~V} 000369$ & 003851.55 & $48 \quad 1712.81$ & 19.54 & - & 163 & 0.32 & 5.1 & - & - & $\mathrm{u}$ & 14120124 \\
\hline $185 \mathrm{~V} 000370$ & 003851.70 & 482100.12 & 19.57 & 16.79 & 303 & 0.41 & 6.9 & - & - & $\mathrm{C}$ & 14151336 \\
\hline 185V000371 & 003848.89 & 482158.67 & 19.92 & 16.98 & 108 & 0.41 & 3.6 & - & - & M & 15671647 \\
\hline $185 \mathrm{~V} 000372$ & 003848.46 & 481824.96 & 19.65 & - & 145 & 0.24 & 5.5 & - & - & $\mathrm{u}$ & - \\
\hline $185 \mathrm{~V} 000373$ & 003848.27 & 481916.59 & 20.53 & - & 98 & 0.74 & 6.1 & - & - & M & 15930783 \\
\hline 185V000374 & 003846.10 & 482028.43 & 19.54 & - & 99 & 0.19 & 4.5 & - & - & M & 17111165 \\
\hline $185 \mathrm{~V} 000375$ & 003845.60 & 481707.01 & 20.11 & - & 178 & 0.46 & 2.5 & - & - & $\mathrm{u}$ & 17280090 \\
\hline $185 \mathrm{~V} 000376$ & 003845.05 & 481946.28 & 19.78 & 17.67 & 462 & 0.25 & 4.6 & - & - & S & 17640940 \\
\hline $185 \mathrm{~V} 000377$ & 003841.45 & 481815.15 & 19.91 & - & 103 & 0.44 & 5.2 & - & - & S & 19520452 \\
\hline 185V000378 & 003857.76 & 482003.72 & 20.78 & 15.48 & 535 & 1.27 & 5.8 & - & - & $\mathrm{u}$ & 10911037 \\
\hline $185 \mathrm{~V} 000379$ & 003852.32 & 481925.86 & 20.91 & 16.75 & 90 & 0.86 & 1.5 & - & - & $\mathrm{u}$ & - \\
\hline $185 \mathrm{~V} 000380$ & 003855.08 & 482040.45 & 19.99 & 16.84 & 168 & 0.42 & 3.0 & - & - & $\mathrm{u}$ & - \\
\hline 185V000381 & 003849.06 & 482122.88 & 19.55 & - & 137 & 0.29 & 3.6 & - & - & M & 15561457 \\
\hline $185 \mathrm{~V} 000382$ & 003900.10 & 482035.47 & 19.63 & 16.22 & 363 & 0.48 & 4.5 & 725 & 2.9 & $\mathrm{C}$ & 09691207 \\
\hline $185 \mathrm{~V} 000383$ & 003904.47 & $48 \quad 1812.81$ & 19.10 & 15.78 & 450 & 1.73 & 6.1 & 899 & 1.1 & $\mathrm{u}$ & - \\
\hline $185 \mathrm{~V} 000384$ & 003903.30 & 481944.34 & 21.32 & 17.25 & 82 & 0.80 & 1.7 & 164 & 4.1 & $\mathrm{~S}$ & 07960936 \\
\hline $185 \mathrm{~V} 000385$ & 003900.01 & 481926.39 & 19.43 & 16.84 & 121 & 0.34 & 2.5 & 243 & 5.1 & $\mathrm{C}$ & 09700839 \\
\hline 185V000386 & 003858.19 & 481929.57 & 19.83 & - & 81 & 0.44 & 2.4 & 161 & 4.9 & M & 10670855 \\
\hline $185 \mathrm{~V} 000387$ & 003856.96 & 482015.41 & 19.53 & 17.16 & 198 & 0.50 & 1.1 & 397 & 3.9 & $\mathrm{u}$ & 11341099 \\
\hline $185 \mathrm{~V} 000388$ & 003856.46 & 481918.01 & 20.59 & - & 60 & 0.45 & 1.4 & 120 & 2.9 & M & 11580793 \\
\hline 185V000389 & 003856.42 & 481944.63 & 20.11 & 16.96 & 118 & 0.60 & 2.2 & 236 & 5.3 & $\mathrm{u}$ & - \\
\hline $185 \mathrm{~V} 000390$ & 003905.52 & 481729.54 & 19.33 & 16.74 & 354 & 1.34 & 5.4 & 1204 & 4.6 & M & 06710219 \\
\hline 185V000391 & 003858.97 & 481742.37 & 19.63 & 17.04 & 279 & 0.76 & 4.3 & 1067 & 3.9 & $\mathrm{C}$ & 10200285 \\
\hline $185 \mathrm{~V} 000392$ & 003851.50 & 482005.26 & 19.08 & 16.00 & 347 & 0.41 & 4.3 & 440 & 3.2 & $\mathrm{C}$ & 14231043 \\
\hline $185 \mathrm{~V} 000393$ & 003900.18 & 481936.15 & 19.56 & 16.89 & 329 & 1.43 & 6.8 & 985 & 3.1 & M & 09610891 \\
\hline $185 \mathrm{~V} 000394$ & 003851.45 & 482115.53 & 19.63 & - & 134 & 0.66 & 4.2 & 437 & 4.7 & M & 14291418 \\
\hline $185 \mathrm{~V} 000395$ & 003848.07 & 482121.79 & 19.78 & 17.60 & 213 & 0.88 & 4.4 & 800 & 3.8 & $\mathrm{C}$ & 16091450 \\
\hline 185V000396 & 003844.76 & 482110.92 & 20.57 & 17.02 & 234 & 1.69 & 5.6 & 472 & 1.0 & $\mathrm{C}$ & 17841392 \\
\hline $185 \mathrm{~V} 000397$ & 003902.92 & 481933.16 & 20.56 & - & 153 & 1.48 & 3.4 & 414 & 1.0 & $\mathrm{~S}$ & 08160876 \\
\hline 185V000398 & 003904.35 & 482100.38 & 19.94 & 16.05 & 427 & 1.07 & 3.0 & 1088 & 0.6 & $\mathrm{u}$ & - \\
\hline $185 \mathrm{~V} 000399$ & 003904.06 & 482043.22 & 21.59 & 17.09 & 406 & 1.79 & 4.2 & 829 & 1.3 & $\mathrm{u}$ & - \\
\hline $185 \mathrm{~V} 000400$ & 003851.26 & 481949.71 & 20.76 & 16.80 & 372 & 1.05 & 4.0 & 664 & 1.5 & $\mathrm{u}$ & - \\
\hline $185 \mathrm{~V} 000401$ & 003843.97 & 481705.80 & 18.17 & - & 185 & 0.59 & 3.1 & 873 & 2.7 & $\mathrm{u}$ & - \\
\hline $185 \mathrm{~V} 000402$ & 003843.78 & 481756.79 & 19.72 & 17.42 & 103 & 0.69 & 1.0 & 205 & 5.2 & $\mathrm{u}$ & - \\
\hline $185 \mathrm{~V} 000403$ & 003900.68 & 482013.99 & 19.74 & 16.10 & 374 & 0.74 & 5.9 & 574 & 2.2 & $\mathrm{u}$ & - \\
\hline $185 \mathrm{~V} 000404$ & 003900.53 & 481934.64 & 20.17 & 15.89 & 367 & 0.86 & 6.5 & 840 & 3.1 & $\mathrm{u}$ & - \\
\hline $185 \mathrm{~V} 000405$ & 003855.52 & 482026.87 & 18.98 & 16.29 & 116 & 0.29 & 2.6 & 325 & 4.1 & $\mathrm{u}$ & - \\
\hline 185V000406 & 003852.07 & 482230.33 & 20.53 & 16.77 & 193 & 1.02 & 3.2 & 234 & 1.8 & $\mathrm{u}$ & - \\
\hline $185 \mathrm{~V} 000407$ & 003912.39 & 482022.12 & 19.66 & - & 110 & 0.43 & 4.3 & 131 & 3.6 & $\mathrm{u}$ & - \\
\hline $185 \mathrm{~V} 000408$ & 003910.98 & 482116.09 & 20.10 & - & 93 & 0.37 & 3.4 & 963 & 3.3 & M & 03941428 \\
\hline 185V000409 & 003903.42 & 481913.30 & 20.23 & 16.83 & 112 & 0.60 & 2.3 & 127 & 4.7 & M & 07880771 \\
\hline $185 \mathrm{~V} 000410$ & 003857.77 & 481943.34 & 19.57 & 18.43 & 98 & 0.19 & 3.2 & 553 & 4.0 & $\mathrm{~S}$ & 10900929 \\
\hline $185 \mathrm{~V} 000411$ & 003856.85 & 481949.68 & 20.05 & 16.23 & 166 & 0.48 & 3.5 & 195 & 4.9 & $\mathrm{u}$ & - \\
\hline $185 \mathrm{~V} 000412$ & 003857.06 & 482118.17 & 19.06 & 15.95 & 197 & 0.28 & 4.5 & 489 & 3.0 & $\mathrm{u}$ & 11321434 \\
\hline $185 \mathrm{~V} 000413$ & 003855.34 & 481927.73 & 19.59 & 16.10 & 132 & 0.27 & 5.5 & 293 & 4.1 & M & 12180845 \\
\hline 185V000414 & 003854.19 & 481959.79 & 19.63 & - & 119 & 0.37 & 4.2 & 749 & 4.6 & $\mathrm{u}$ & - \\
\hline $185 \mathrm{~V} 000415$ & 003853.77 & 481914.80 & 19.46 & 16.46 & 171 & 0.28 & 3.2 & 236 & 3.8 & $\mathrm{u}$ & - \\
\hline $185 \mathrm{~V} 000416$ & 003852.64 & 482116.71 & 19.88 & - & 122 & 0.36 & 3.7 & 416 & 4.3 & $\mathrm{~S}$ & 13661425 \\
\hline $185 \mathrm{~V} 000417$ & 003852.37 & 481913.63 & 20.51 & 16.64 & 122 & 0.52 & 2.6 & 865 & 4.5 & $\mathrm{u}$ & - \\
\hline 185V000418 & 003852.03 & 482131.35 & 19.79 & - & 90 & 0.33 & 3.2 & 397 & 6.5 & $\mathrm{~S}$ & 14001503 \\
\hline $185 \mathrm{~V} 000419$ & 003851.41 & 481837.93 & 19.83 & 17.17 & 104 & 0.35 & 3.0 & 911 & 4.7 & M & 14240578 \\
\hline
\end{tabular}


Table A.2. continued.

\begin{tabular}{|c|c|c|c|c|c|c|c|c|c|c|c|}
\hline ID & $\begin{array}{c}\text { RAJ2000 } \\
\text { h:m:s }\end{array}$ & $\begin{array}{c}\text { DEJ2000 } \\
\text { d:m:s }\end{array}$ & $\left.\begin{array}{c}i \\
{[\mathrm{mag}]}\end{array}\right]$ & $\left.\begin{array}{c}K_{\mathrm{s}} \\
{[\mathrm{mag}]}\end{array}\right]$ & $\begin{array}{l}P_{0} \\
{[\mathrm{~d}]}\end{array}$ & $\begin{array}{c}\Delta i \\
{[\mathrm{mag}]}\end{array}$ & $s i g_{0}$ & $\begin{array}{l}P_{1} \\
{[\mathrm{~d}]}\end{array}$ & $s i g_{1}$ & type & ID ${ }_{\text {Paper I }}$ \\
\hline $185 \mathrm{~V} 000420$ & 003913.96 & 481820.15 & 20.24 & - & - & 0.43 & - & - & - & $\mathrm{S}$ & $\overline{02260493}$ \\
\hline 185V000421 & 003913.33 & 482023.69 & 19.03 & 16.96 & - & 0.34 & - & - & - & $\mathrm{S}$ & 02661150 \\
\hline $185 \mathrm{~V} 000422$ & 003841.24 & 482024.20 & 19.72 & - & - & 0.47 & - & - & - & $\mathrm{S}$ & 19691141 \\
\hline $185 \mathrm{~V} 000423$ & 003910.02 & 482225.13 & 20.48 & - & - & 0.64 & - & - & - & $\mathrm{u}$ & 04481795 \\
\hline $185 \mathrm{~V} 000424$ & 003904.25 & 482104.40 & 19.72 & - & - & 0.52 & - & - & - & $\mathrm{S}$ & 07501363 \\
\hline $185 \mathrm{~V} 000425$ & 003902.12 & 482029.88 & 19.91 & 17.29 & - & 0.35 & - & - & - & M & 08611178 \\
\hline $185 \mathrm{~V} 000426$ & 003900.38 & 482005.65 & 19.44 & 16.54 & - & 0.23 & - & - & - & $\mathrm{C}$ & 09521049 \\
\hline $185 \mathrm{~V} 000427$ & 003859.83 & 482111.05 & 19.64 & 17.30 & - & 0.35 & - & - & - & M & 09851397 \\
\hline $185 \mathrm{~V} 000428$ & 003859.66 & 482025.67 & 20.99 & 16.75 & - & 0.87 & - & - & - & $\mathrm{C}$ & 09921155 \\
\hline $185 \mathrm{~V} 000429$ & 003858.56 & 482140.59 & 19.94 & 17.83 & - & 0.28 & - & - & - & M & 10541554 \\
\hline $185 \mathrm{~V} 000430$ & 003857.37 & 481959.50 & 20.24 & 17.30 & - & 0.92 & - & - & - & $\mathrm{S}$ & 11111015 \\
\hline $185 \mathrm{~V} 000431$ & 003855.17 & 482054.15 & 19.10 & - & - & 0.35 & - & - & - & $\mathrm{S}$ & 12311305 \\
\hline $185 \mathrm{~V} 000432$ & 003854.94 & 482041.41 & 19.76 & 16.77 & - & 0.38 & - & - & - & $\mathrm{u}$ & - \\
\hline $185 \mathrm{~V} 000433$ & 003854.79 & 482119.01 & 19.92 & 17.38 & - & 0.74 & - & - & - & M & 12521438 \\
\hline $185 \mathrm{~V} 000434$ & 003853.24 & 481851.51 & 19.49 & 17.09 & - & 0.24 & - & - & - & $\mathrm{C}$ & 13270651 \\
\hline $185 \mathrm{~V} 000435$ & 003849.41 & 481940.18 & 19.52 & 17.49 & - & 0.31 & - & - & - & M & 15330909 \\
\hline 185V000436 & 003849.20 & 482006.23 & 19.55 & 17.15 & - & 0.41 & - & - & - & M & 15451048 \\
\hline 185V000437 & 003845.95 & 481747.61 & 19.75 & - & - & 0.32 & - & - & - & M & 17120307 \\
\hline 185V000438 & 003856.84 & 481850.58 & 21.32 & 16.80 & - & 1.45 & - & - & - & $\mathrm{C}$ & 11360647 \\
\hline $185 \mathrm{~V} 000439$ & 003855.32 & 482001.70 & 20.11 & 16.66 & - & 0.66 & - & - & - & M & 12211026 \\
\hline $185 \mathrm{~V} 000440$ & 003854.45 & 482049.69 & 20.95 & - & - & 1.24 & - & - & - & $\mathrm{S}$ & 12691281 \\
\hline 185V000441 & 003906.45 & 482052.64 & 19.52 & 16.78 & - & 0.46 & - & - & - & $\mathrm{u}$ & - \\
\hline 185V000442 & 003901.54 & 482110.56 & 19.80 & 16.83 & - & 0.63 & - & - & - & $\mathrm{u}$ & - \\
\hline $185 \mathrm{~V} 000443$ & 003857.97 & 482058.10 & 19.64 & 17.38 & - & 0.43 & - & - & - & $\mathrm{u}$ & - \\
\hline 185V000444 & 003856.04 & 481804.53 & 19.76 & 17.14 & - & 0.44 & - & - & - & $\mathrm{u}$ & - \\
\hline $185 \mathrm{~V} 000445$ & 003855.83 & 481915.82 & 20.50 & 17.01 & - & 0.75 & - & - & - & $\mathrm{u}$ & - \\
\hline 185V000446 & 003854.99 & 481900.60 & 19.90 & 16.70 & - & 0.48 & - & - & - & $\mathrm{u}$ & - \\
\hline 185V000447 & 003851.60 & 481906.70 & 20.01 & 16.62 & - & 0.60 & - & - & - & $\mathrm{u}$ & - \\
\hline 185V000448 & 003851.43 & 482017.92 & 20.33 & 16.88 & - & 0.75 & - & - & - & $\mathrm{u}$ & - \\
\hline 185V000449 & 003859.82 & 482036.92 & 20.23 & - & - & 0.50 & - & - & - & $\mathrm{u}$ & - \\
\hline $185 \mathrm{~V} 000450$ & 003857.74 & 481954.99 & 19.61 & 17.26 & - & 0.41 & - & - & - & $\mathrm{u}$ & - \\
\hline $185 \mathrm{~V} 000451$ & 003857.65 & 482025.18 & 19.69 & 17.51 & - & 0.48 & - & - & - & $\mathrm{u}$ & - \\
\hline $185 \mathrm{~V} 000452$ & 003915.30 & 482124.61 & 20.11 & - & - & 0.37 & - & - & - & $\mathrm{S}$ & 01651474 \\
\hline $185 \mathrm{~V} 000453$ & 003913.68 & 482123.58 & 20.44 & 16.90 & - & 1.05 & - & - & - & $\mathrm{C}$ & 02511468 \\
\hline $185 \mathrm{~V} 000454$ & 003912.29 & 482222.82 & 19.58 & - & - & 0.33 & - & - & - & $\mathrm{S}$ & 03281783 \\
\hline 185V000455 & 003910.59 & 482157.83 & 19.74 & - & - & 0.32 & - & - & - & $\mathrm{S}$ & 04171650 \\
\hline $185 \mathrm{~V} 000456$ & 003910.41 & 481931.99 & 19.80 & - & - & 0.49 & - & - & - & M & 04180873 \\
\hline $185 \mathrm{~V} 000457$ & 003907.73 & 481957.15 & 20.26 & - & - & 0.38 & - & - & - & M & 05621006 \\
\hline 185V000458 & 003907.45 & 482129.86 & 19.65 & - & - & 0.39 & - & - & - & M & 05821500 \\
\hline $185 \mathrm{~V} 000459$ & 003906.89 & 481824.18 & 20.83 & - & - & 0.69 & - & - & - & $\mathrm{u}$ & 06010511 \\
\hline $185 \mathrm{~V} 000460$ & 003906.88 & 481852.43 & 18.61 & 15.44 & - & 1.13 & - & - & - & $\mathrm{u}$ & - \\
\hline 185V000461 & 003906.88 & 482237.38 & 19.50 & 17.23 & - & 0.24 & - & - & - & M & 06151859 \\
\hline $185 \mathrm{~V} 000462$ & 003906.71 & 482044.67 & 19.39 & 16.65 & - & 0.32 & - & - & - & $\mathrm{u}$ & - \\
\hline $185 \mathrm{~V} 000463$ & 003904.62 & 482010.86 & 20.00 & 17.09 & - & 0.40 & - & - & - & $\mathrm{u}$ & - \\
\hline 185V000464 & 003903.37 & 482047.83 & 20.40 & - & - & 0.39 & - & - & - & $\mathrm{S}$ & 07961274 \\
\hline $185 \mathrm{~V} 000465$ & 003903.11 & 481903.15 & 20.11 & 17.43 & - & 0.47 & - & - & - & M & 08040717 \\
\hline 185V000466 & 003903.08 & 482204.28 & 19.76 & 17.28 & - & 0.28 & - & - & - & M & 08151681 \\
\hline 185V000467 & 003902.87 & 482042.50 & 20.55 & 17.30 & - & 0.52 & - & - & - & M & 08221246 \\
\hline 185V000468 & 003902.81 & 482014.76 & 19.16 & 16.99 & - & 0.20 & - & - & - & M & 08241098 \\
\hline 185V000469 & 003902.69 & 481854.96 & 21.99 & - & - & 1.36 & - & - & - & $\mathrm{u}$ & - \\
\hline $185 \mathrm{~V} 000470$ & 003902.50 & 482110.82 & 19.81 & - & - & 0.26 & - & - & - & M & 08431397 \\
\hline 185V000471 & 003902.15 & & & 16.71 & - & 0.42 & - & - & - & $\mathrm{S}$ & 08560749 \\
\hline $185 \mathrm{~V} 000472$ & 003901.98 & 482008.93 & 19.03 & 16.80 & - & 0.21 & - & - & - & $\mathrm{u}$ & - \\
\hline $185 \mathrm{~V} 000473$ & 003901.80 & 481944.05 & 21.32 & 16.95 & - & 1.10 & - & - & - & $\mathrm{u}$ & - \\
\hline 185V000474 & 003901.77 & 482016.48 & 21.36 & - & - & 0.85 & - & - & - & $\mathrm{u}$ & - \\
\hline 185V000475 & 003901.72 & 482255.08 & 19.69 & 17.78 & - & 0.28 & - & - & - & $\mathrm{S}$ & 08901952 \\
\hline 185V000476 & 003901.36 & 482022.39 & 20.39 & 17.18 & - & 0.53 & - & - & - & $\mathrm{u}$ & - \\
\hline 185V000477 & 003901.19 & 482047.22 & 20.59 & - & - & 0.55 & - & - & - & M & 09111270 \\
\hline 185V000478 & 003901.14 & 482130.87 & 19.44 & - & - & 0.30 & - & - & - & $\mathrm{S}$ & 09161503 \\
\hline 185V000479 & 003900.74 & 481826.45 & 21.75 & - & - & 0.98 & - & - & - & $\mathrm{u}$ & 09280520 \\
\hline
\end{tabular}


Table A.2. continued.

\begin{tabular}{|c|c|c|c|c|c|c|c|c|c|c|c|}
\hline ID & $\begin{array}{l}\text { RAJ2000 } \\
\text { h:m:s }\end{array}$ & $\begin{array}{c}\text { DEJ2000 } \\
\text { d:m:s }\end{array}$ & $\begin{array}{c}i \\
{[\mathrm{mag}]}\end{array}$ & $\begin{array}{c}K_{\mathrm{s}} \\
{[\mathrm{mag}]}\end{array}$ & $\begin{array}{l}P_{0} \\
{[\mathrm{~d}]}\end{array}$ & $\begin{array}{c}\Delta i \\
{[\mathrm{mag}]}\end{array}$ & $s i g_{0}$ & $\begin{array}{l}P_{1} \\
{[\mathrm{~d}]}\end{array}$ & $\operatorname{sig}_{1}$ & type & ID Paper I \\
\hline $185 \mathrm{~V} 000480$ & 003900.80 & 482051.49 & 20.64 & - & - & 0.46 & - & - & - & $\bar{M}$ & 09321293 \\
\hline 185V000481 & 003900.10 & 481727.80 & 19.86 & 17.33 & - & 0.59 & - & - & - & M & 09590207 \\
\hline $185 \mathrm{~V} 000482$ & 003859.09 & 482050.51 & 19.75 & 17.37 & - & 0.44 & - & - & - & $\mathrm{u}$ & - \\
\hline $185 \mathrm{~V} 000483$ & 003858.86 & 482013.25 & 18.70 & 15.50 & - & 0.23 & - & - & - & $\mathrm{u}$ & - \\
\hline 185V000484 & 003858.76 & 482222.87 & 19.17 & 16.41 & - & 0.26 & - & - & - & $\mathrm{C}$ & 10451779 \\
\hline 185V000485 & 003858.47 & 482018.18 & 20.44 & 16.93 & - & 1.06 & - & - & - & $\mathrm{u}$ & - \\
\hline 185V000486 & 003858.13 & 482019.74 & 19.91 & 16.38 & - & 0.68 & - & - & - & M & 10721123 \\
\hline 185V000487 & 003857.95 & 482014.51 & 19.46 & 16.01 & - & 0.51 & - & - & - & $\mathrm{u}$ & - \\
\hline 185V000488 & 003857.77 & 482008.43 & 19.77 & 16.47 & - & 0.48 & - & - & - & $\mathrm{u}$ & - \\
\hline 185V000489 & 003857.72 & 482034.95 & 20.15 & - & - & 0.46 & - & - & - & $\mathrm{u}$ & - \\
\hline $185 \mathrm{~V} 000490$ & 003857.60 & 482021.92 & 20.35 & 16.01 & - & 0.84 & - & - & - & $\mathrm{u}$ & - \\
\hline 185V000491 & 003857.11 & 481719.14 & 19.91 & 17.14 & - & 0.47 & - & - & - & $\mathrm{u}$ & 11180160 \\
\hline $185 \mathrm{~V} 000492$ & 003857.21 & 482026.79 & 19.26 & 16.21 & - & 0.31 & - & - & - & $\mathrm{u}$ & - \\
\hline 185V000493 & 003857.11 & 481924.40 & 20.77 & 17.09 & - & 0.64 & - & - & - & $\mathrm{u}$ & 11240828 \\
\hline 185V000494 & 003857.02 & 482144.51 & 20.81 & - & - & 0.52 & - & - & - & $\mathrm{S}$ & 11351574 \\
\hline 185V000495 & 003856.81 & 481953.50 & 21.09 & 16.85 & - & 1.32 & - & - & - & $\mathrm{u}$ & - \\
\hline 185V000496 & 003856.43 & 481950.15 & 19.46 & 16.11 & - & 0.28 & - & - & - & $\mathrm{C}$ & 11610965 \\
\hline 185V000497 & 003856.41 & 482055.93 & 19.73 & - & - & 0.51 & - & - & - & M & 11651315 \\
\hline 185V000498 & 003856.13 & 481953.30 & 21.46 & 16.76 & - & 1.00 & - & - & - & $\mathrm{u}$ & 11770981 \\
\hline 185V000499 & 003855.91 & 481745.91 & 21.61 & - & - & 1.34 & - & - & - & $\mathrm{u}$ & - \\
\hline $185 \mathrm{~V} 000500$ & 003855.82 & 481943.96 & 19.60 & 16.72 & - & 0.36 & - & - & - & $\mathrm{u}$ & 11930931 \\
\hline 185V000501 & 003855.68 & 482021.70 & 19.76 & 16.74 & - & 0.36 & - & - & - & $\mathrm{u}$ & - \\
\hline 185V000502 & 003855.22 & 481939.14 & 19.76 & 16.98 & - & 0.43 & - & - & - & $\mathrm{C}$ & 12240905 \\
\hline $185 \mathrm{~V} 000503$ & 003854.95 & 482041.27 & 19.74 & 16.76 & - & 0.37 & - & - & - & $\mathrm{u}$ & - \\
\hline 185V000504 & 003854.50 & 481933.95 & 19.44 & 16.41 & - & 0.42 & - & - & - & $\mathrm{C}$ & 12620877 \\
\hline 185V000505 & 003854.37 & 482232.96 & 20.75 & 17.74 & - & 0.68 & - & - & - & $\mathrm{S}$ & 12781832 \\
\hline $185 \mathrm{~V} 000506$ & 003854.22 & 482018.55 & 20.71 & 16.99 & - & 1.11 & - & - & - & $\mathrm{u}$ & - \\
\hline 185V000507 & 003852.70 & 482122.57 & 19.98 & 16.38 & - & 0.40 & - & - & - & $\mathrm{u}$ & 13631456 \\
\hline 185V000508 & 003852.56 & 481938.45 & 19.63 & 16.67 & - & 0.29 & - & - & - & $\mathrm{u}$ & - \\
\hline 185V000509 & 003847.95 & 482224.99 & 21.63 & - & - & 0.91 & - & - & - & $\mathrm{u}$ & - \\
\hline $185 \mathrm{~V} 000510$ & 003847.26 & 481852.42 & 19.59 & - & - & 0.35 & - & - & - & $\mathrm{S}$ & 16450653 \\
\hline 185V000511 & 003846.32 & 481854.72 & 22.33 & - & - & 1.49 & - & - & - & M & 16950665 \\
\hline $185 \mathrm{~V} 000512$ & 003845.09 & 481850.49 & 19.79 & - & - & 0.31 & - & - & - & M & 17600642 \\
\hline $185 \mathrm{~V} 000513$ & 003845.18 & 482043.02 & 20.36 & 16.86 & - & 0.37 & - & - & - & $\mathrm{S}$ & 17601243 \\
\hline
\end{tabular}

